\title{
Natural Nitrogenous Sesquiterpenoids and Their Bioactivity: A Review
}

\author{
De-Li Chen ${ }^{1,2} \oplus$, Bo-Wen Wang ${ }^{3}$, Zhao-Cui Sun ${ }^{1}$, Jun-Shan Yang ${ }^{1}$, Xu-Dong $\mathrm{Xu}^{1, *}$ and \\ Guo-Xu Ma 1,2,* \\ 1 Institute of Medicinal Plant Development, Chinese Academy of Medical Sciences \& Peking Union \\ Medical College, No. 151, Malianwa North Road, Haidian District, Beijing 100193, China; \\ chendeli9999@163.com (D.-L.C.); flydancingsun@163.com (Z.-C.S.); jsyang@implad.ac.cn (J.-S.Y.) \\ 2 Hainan Branch of Institute of Medicinal Plant Development, Chinese Academy of Medicinal Sciences \& \\ Peking Union Medical College (Hainan Provincial Key Laboratory of Resources Conservation and \\ Development of Southern Medicine), Haikou 570311, China \\ 3 School of Chemical Engineering and Technology, Hainan University, Haikou 570228, China; \\ 13699719479@163.com \\ * Correspondence: xdxu@implad.ac.cn (X.-D.X.); gxma@implad.ac.cn (G.-X.M.); \\ Tel.: +86-010-57833296 (X.-D.X.)
}

Academic Editor: Anna Carbone

Received: 6 May 2020; Accepted: 22 May 2020; Published: 27 May 2020

\begin{abstract}
Nitrogenous sesquiterpenoids from natural sources are rare, so unsurprisingly neither the potentially valuable bioactivity nor the broad structural diversity of nitrogenous sesquiterpenoids has been reviewed before. This report covers the progressd uring the decade from 2010 to February 2020 on the isolation, identification, and bioactivity of 391 nitrogen-containing natural sesquiterpenes from terrestrial plant, marine organisms, and microorganisms. This complete and in-depth review should be helpful for discovering and developing new drugs of medicinal value related to natural nitrogenous sesquiterpenoids.
\end{abstract}

Keywords: nitrogenous sesquiterpenoids; celastraceae; marine sponge; fungi; bioactivities

\section{Introduction}

The natural products commonly termed 'secondary metabolites' in contrast to 'primary metabolites', are produced byorganisms in order to provide an evolutionary benefit [1]. Natural products as a major chemical resource, have played a significant role over the last 200 years in treating and preventing diseases, and continue to serve as important agents in modern drug discovery due to their characteristic chemical spatial orientation, which enables them tointeract with their natural and other biological targets [1-4]. Recently, half of new drugs reported were naturally occurring or constructed on the basis of some natural chemical framework [4-6].

Sesquiterpenoids are the largest class of natural terpenoids, with a structural diversity that includes thousands of compounds and more than 100 skeletal types [7]. Many of them show 'drug-like' chemical properties, including alkylating center reactivity, lipophilicity, and favorable molecular geometry and electronic features, and have attracted considerable interest due to their pronounced biological activities [8,9]. Meanwhile, sesquiterpenoids that contain nitrogen bonds constitute a fascinating group with enormous structural diversity [10]. Interestingly, it is notable that nitrogenous sesquiterpenoids are rare innatural sources, and there are only a few hundred such compounds that contain the element $\mathrm{N}$ known to be produced bycertain species. Functionally and biologically important to humans, have caught the attention of a number of scientists, and extensive phytochemical and biological investigations of nitrogenous sesquiterpenoids from natural sources have been carried out by researchers at the recent ten years [10-12]. 
While the scientific community is generally aware of the rarity of the $\mathrm{N}$ bond in natural sequiterpenoids, and there are many reviews providing extensive coverage on sesquiterpenoids [11,12], including the naturally occurring disesquiterpenoids $[1,13,14]$, natural products containing a nitrogen-nitrogen bond [15] or nitrogen-sulfur bond [16], neither the potentially valuable bioactivity nor the broad structural diversity of nitrogenous sesquiterpenoids has been systematically reviewed during the past ten years.

In this review, nitrogenous sesquiterpenoids from biological sources, including plants, microorganisms, and marine resources, will be considered. In order to be as comprehensive and clear as possible, the natural nitrogenous sesquiterpenoids have been segregated by structural class and compounds covered in the past decade included where appropriate. This report provides a systematic review of the isolation, structural characterization and biological activities of these compounds since 2010, if known.

\section{Species Containing Nitrogenous Sesquite Rpenoids and Their Bioactivities}

\subsection{Dihydroagar of Uran Sesquiterpenoids}

Nitrogen-containing dihydroagarofuran sesquiterpenoids feature several ester groups on a highly oxygenated tricyclic scaffold, and their polyesterified macrolide sesquiterpenoid pyridine alkaloids possess a characteristic macrocyclic dilactone skeleton consisting of a dicarboxy licacid moiety, 2-(carboxyalkyl)nicotinic acid, and a polyoxygenated dihydro- $\beta$-agarofuran sesquiterpenoid (Figure 1 and Table 1). The hydroxyl groups of the latter are usually esterified by various organicacids including acetic, benzoic, furanoic, nicotinic, and cinnamicacids. The 2-(carboxyalkyl)nicotinic acid moiety originates from evoninic acid, wilfordic acid, hydroxywilfordic acid, ortheir congeners. The number, position, and configuration of these substituents create a largenovel chemical diversity and exhibit abroad range of biological activities.

Dihydroagarofuran sesquiterpenoids were considered the most widespread and characteristic metabolites of the plants of the Celastraceae. Compounds 1-12 were isolated from the roots of Maytenus mekongensis [17]. Compounds 1-5 having wilfordic acid moieties, either with or without a $9^{\prime}$-OAc group, exhibited comparable antiplasmodial activities, with $\mathrm{IC}_{50}$ values of $3.1 \times 10^{-3}$, $3.9 \times 10^{-3}, 3.5 \times 10^{-3}, 3.1 \times 10^{-3}$ and $2.5 \times 10^{-3} \mathrm{mM}$ respectively, while compounds $\mathbf{1 0}-\mathbf{1 2}$ with evoninic acid moieties showed no inhibitory activity. Compounds 12-29 were extracted from the dried roots of Tripterygium wilfordii [18]. Compound 22 displayed 22.3\% inhibitory activity against HSV2 in vitro at $0.5 \mathrm{mg} / \mathrm{mL}$, and acyclovir $66.3 \%$ inhibitory activity at $0.5 \mathrm{mg} / \mathrm{mL}$. Compound 28 showed $31.7 \%$ inhibitory activity at $0.25 \mathrm{mg} / \mathrm{mL}$, while acyclovir displayed $60.6 \%$ inhibitory activity at $0.25 \mathrm{mg} / \mathrm{mL}$. Compounds 30 and 31 were obtained from the fruits of Celastrus orbiculatus Thunb [19]. Hypoglaunines E (32) and F (33) have been purified from the root barks of Tripterygium hypoglaucum and showed no cytotoxic activities against five cancer celllines [20]. Triptersinines A-H, L (compounds 34-42), peritassine A (26), wilfordinine A (43), hypoglaunine A (44), hypoglaunine E (32), wilfordinine E (45), euonine (46), wilfortrine (21), euonymine (12) were extracted from the leaves of Tripterygium wilfordii, and compounds $26,34,43$, and 46 showed moderate inhibitory effects onnitric oxide production in LPS-induced macrophages at $5 \mu \mathrm{M}$ [21]. Compounds 47-49 were identified from thestems of Euonymus alatus [22]. Triptersinines M-T (compounds 50-57) and wilforgine (18) have been extracted from the the leaves of Tripterygium wilfordii, and compounds 50,51, 54, 57, and 18 showed moderate inhibitory abilities on NO production and no influence on cell viability by the MTT method, the other compounds exhibited weak effects [23]. Compounds 7, 25, 58-91 were obtained from the dried roots of Tripterygium wilfordii [24]. Tripterygiumine Q (81) exhibited immunosuppressive activity with an $\mathrm{IC}_{50}$ value of $8.67 \mu \mathrm{M}$, and no cytotoxicity was observed even at a dose of $100 \mu \mathrm{M}$. Triptonine $\mathrm{B}$ (82) not only exhibited immunosuppressive activity with an $\mathrm{IC}_{50}$ value of $4.95 \mu \mathrm{M}$, but also showed cytotoxicity with an $\mathrm{IC}_{50}$ value of $26.41 \mu \mathrm{M}$. Compounds 92-95 were isolated from the leaves of Maytenus spinosa [25], and the isolates displayed no anti-HIV activity. Tripterygiumines S-W (96-100), 
wilfornine A (101), wilfornine D (102), tripfordine A (103), 2-debenzoyl-2-nicotinoylwilforine (104) along with 12-13, 18-20, 25, 75, and 87 were purified from the roots of the Tripterygium wilfordii, and found that 13 and $\mathbf{9 6}$ possessed potent nitric oxide inhibitory activity with $\mathrm{IC}_{50}$ values ranging from 2.99 to $28.80 \mu \mathrm{M}$, without any effect on the cell viability of RAW 264.7 cells [26]. Accordingly, compounds 13 and 96, especially 13, were identified as promising candidates for further scientific investigation of their potential use as anti-inflammatory agents. Compound $\mathbf{1 0 5}$ was obtained from the whole plants of Parnassia wightiana, and showed some cytotoxic activities against NB4, MKN-45 and MCF-7 cells at $20 \mu \mathrm{M}$ [27]. Triptregelines A-J (106-115), regelidine (28), $1 \alpha, 6 \beta, 15$-triacetoxy- $8 \alpha$-benzoyloxy-4 $\beta$-hydroxyl-9 $\alpha$-(3-nicotinoyloxy)-dihydro- $\beta$-agarofuran (116), dimacroregeline A-B (117-118) and triptonine A (119) have been isolated from the stems of Tripterygium regelii, and 107, 108, 113 and 116 exhibited weak cytotoxic effects on taxol-resistant A549T with $\mathrm{IC}_{50}$ values ranged from 29.4 to $54.4 \mu \mathrm{M}$ [28], 118 showed inhibitory effects on the proliferation of human rheumatoid arthritis synovial fibroblast cell (MH7A) at a concentration of $20 \mu \mathrm{M}$ [29]. Compounds 120-123 were extracted from the stems of Maytenusoblongata [30]. 1-O-Benzoyl-1-deacetyl-4-deoxyalatamine (121) and 1,2-O-dibenzoyl-1,2-deacetyl-4-deoxyalatamine (122) exhibited strong larvicidal activity on the A. aegypti Paea strain with $\mathrm{LD}_{50}$ values of $9.4(95 \% \mathrm{CI}$ : 6.5-10.0) and $2.7 \mu \mathrm{M}(95 \%$ CI: 1.9-2.9), respectively. Triptersinine U (124), hypoglaunine B (125) together with 26, 32, 33, 43, 44, and 46 were isolated from the roots of Tripterygium wilfordii, but all dihydroagarofuran derivatives didn't show cytotoxicity against six human tumor celllines (HepG2, Hep3B, Bcap37, U251, MCF-7 and A549) [31]. Neuroprotective triptersinine Z4-Z14 (126-130, 132-137) and euojaponine $C$ (131) have been obtained from the leaves of Tripterygium wilfordii [32,33], and 126, 127, 129-131 increased cell viability of the okadaic acid-treated PC12 cells from $60.4 \pm 23.0 \%$ to $72.4 \pm 14.1$, $71.5 \pm 11.5,75.7 \pm 15.6,81.2 \pm 13.1$, and $86.2 \pm 25.5 \%$ at $10 \mu \mathrm{M}$, respectively [32]. At $10 \mu \mathrm{mol} / \mathrm{L}$, compounds 132 and $\mathbf{1 3 3}$ showed moderate inhibitory effects on NO production in LPS-induced macrophages with inhibitory rate at $31.2 \pm 3.6$ and $40.9 \pm 4.3$ [33]. Two new sesquiterpene pyridine alkaloids, Chinese bittersweet alkaloid A (138) and Chinese bittersweet alkaloid B (139) were isolated from the rootbarks of Celastrus angulatus [34]. Monimins I (140) and II (141) have been extracted from the leaves of Monimopetalum chinense [35]. Tripteryford C (142) and tripteryford E (143) have been obtained from the leaves of Tripterygium wilfordii, and $\mathbf{1 4 2}$ exhibited the better protective activity against human neuroblastoma SH-SY5Y cell injury induced by $\mathrm{H}_{2} \mathrm{O}_{2}$ with $76.63 \%$ cell viability comparing with the positive control Trolox (69.84\%) at $12.5 \mu \mathrm{M}$ [36]. Celaspaculin G (144) was purified fromthe seeds of Celastrus paniculatus, and with non lifespan-extending effect on the nematode Caenorhabditis elegans [37]. 
Table 1. Reported structures ofdihydroagarofuran sesquiterpenoids 1-144.

\begin{tabular}{|c|c|c|c|c|c|c|c|c|c|c|c|c|}
\hline No & Name & $\mathbf{R}_{\mathbf{1}}$ & $\mathbf{R}_{\mathbf{2}}$ & $\mathbf{R}_{3}$ & $\mathbf{R}_{4}$ & $\mathbf{R}_{\mathbf{5}}$ & $\mathbf{R}_{6}$ & $\mathbf{R}_{7}$ & $\mathbf{R}_{8}$ & $\mathbf{R}_{\mathbf{9}}$ & Type & Ref \\
\hline 1 & Mekongensine & OAc & $\mathrm{OBz}$ & $\beta O A c$ & $\beta O A c$ & OAc & $\mathrm{OH}$ & $\beta O A c$ & OAc & $\mathrm{H}$ & A & [17] \\
\hline 2 & 7-epi-Mekongensine & OAc & $\mathrm{OBz}$ & $\alpha \mathrm{OAc}$ & $\beta \mathrm{OAc}$ & OAc & $\mathrm{OH}$ & $\beta \mathrm{OAc}$ & OAc & $\mathrm{H}$ & A & [17] \\
\hline 3 & 1-O-Benzoyl-1-deacetylmekongensine & $\mathrm{OBz}$ & $\mathrm{OBz}$ & $\beta O A c$ & BOAc & OAc & $\mathrm{OH}$ & $\beta O A c$ & OAc & $\mathrm{H}$ & A & {$[17]$} \\
\hline 4 & 9'-Deacetoxymekongensine & OAc & $\mathrm{OBz}$ & $\beta O A c$ & $\beta O A c$ & OAc & $\mathrm{OH}$ & $\beta O A c$ & $\mathrm{H}$ & $\mathrm{H}$ & A & [17] \\
\hline 5 & $\begin{array}{l}\text { 1-O-Benzoyl-1-deacetyl- } \\
\text { 9'-deacetoxymekongensine }\end{array}$ & $\mathrm{OBz}$ & $\mathrm{OBz}$ & $\beta O A c$ & $\beta O A c$ & OAc & $\mathrm{OH}$ & $\beta \mathrm{OAc}$ & $\mathrm{H}$ & $\mathrm{H}$ & A & {$[17]$} \\
\hline 6 & 7-epi-Euojaponine A & OBz & $\mathrm{OH}$ & $\alpha \mathrm{OAc}$ & $\beta \mathrm{OAc}$ & OAc & $\mathrm{OH}$ & $\beta O A c$ & $\mathrm{H}$ & $\mathrm{CH}_{3}$ & $\mathrm{~B}$ & [17] \\
\hline 7 & 2-O-Benzoyl-2- deacetylmayteine & $\mathrm{OBz}$ & OAc & $\beta O A c$ & $\beta \mathrm{OAc}$ & OAc & $\mathrm{OH}$ & $\beta \mathrm{OBz}$ & $\mathrm{H}$ & $\mathrm{CH}_{3}$ & B & {$[17,24]$} \\
\hline 8 & 7-epi-5-O-Benzoyl-5- deacetylperitassine A & OAc & $\mathrm{OBz}$ & $\alpha \mathrm{OAc}$ & $\beta O A c$ & OAc & $\mathrm{OH}$ & $\beta \mathrm{OAc}$ & $\mathrm{H}$ & $\mathrm{CH}_{3}$ & $\mathrm{C}$ & [17] \\
\hline 9 & 7-epi-Euonymine & OAc & OAc & $\alpha \mathrm{OAc}$ & $\beta O A c$ & OAc & $\mathrm{OH}$ & $\beta O A c$ & $\mathrm{H}$ & $\mathrm{CH}_{3}$ & $\mathrm{~B}$ & {$[17]$} \\
\hline 10 & Mayteine & $\mathrm{OBz}$ & OAc & $\beta O A c$ & $\beta O A c$ & OAc & $\mathrm{OH}$ & $\beta O A c$ & $\mathrm{H}$ & $\mathrm{CH}_{3}$ & $\mathrm{~B}$ & {$[17]$} \\
\hline 11 & 7-epi-Mayteine & $\mathrm{OBz}$ & OAc & $\alpha \mathrm{OAc}$ & $\beta \mathrm{OAc}$ & OAc & $\mathrm{OH}$ & $\beta \mathrm{OAc}$ & $\mathrm{H}$ & $\mathrm{CH}_{3}$ & B & [17] \\
\hline 12 & Euonymine & OAc & OAc & $\beta \mathrm{OAc}$ & $\beta O A c$ & OAc & $\mathrm{OH}$ & $\beta O A c$ & $\mathrm{H}$ & $\mathrm{CH}_{3}$ & $\mathrm{~B}$ & {$[17,18,21,26]$} \\
\hline 13 & 9'-O-Acetyl-7-deacetoxy-7-oxowilfortrine & OAc & OAc & $\mathrm{O}$ & $\beta \mathrm{OAc}$ & OAc & $\mathrm{OH}$ & $\beta \mathrm{OFu}$ & OAc & $\mathrm{H}$ & A & {$[18,26]$} \\
\hline 14 & 9'-O-Acetylwilfortrine & OAc & OAc & $\beta O A c$ & $\beta O A c$ & OAc & $\mathrm{OH}$ & $\beta \mathrm{OFu}$ & OAc & $\mathrm{H}$ & A & [18] \\
\hline 15 & 9'-O-Furanoylwilfordine & OAc & $\mathrm{OAc}$ & BOAc & BOAc & OAc & $\mathrm{OH}$ & $\beta \mathrm{OBz}$ & $\mathrm{OFu}$ & $\mathrm{H}$ & A & [18] \\
\hline 16 & 7-O-Benzoyl-5,7-dideacetylwilformine & OAc & $\mathrm{OH}$ & $\beta \mathrm{OBz}$ & $\beta O A c$ & OAc & $\mathrm{OH}$ & $\beta \mathrm{OAc}$ & $\mathrm{H}$ & $\mathrm{H}$ & A & [18] \\
\hline 17 & Wilfortrine & OAc & $\mathrm{OAc}$ & $\beta O A c$ & $\beta O A c$ & OAc & $\mathrm{OH}$ & $\beta \mathrm{OFu}$ & $\mathrm{OH}$ & $\mathrm{H}$ & A & {$[18,21,26]$} \\
\hline 18 & Wilforgine & OAc & OAc & $\beta O A c$ & $\beta O A c$ & OAc & $\mathrm{OH}$ & $\beta \mathrm{OFu}$ & $\mathrm{H}$ & $\mathrm{H}$ & A & {$[18,23,26]$} \\
\hline 19 & Wilfordine & OAc & OAc & $\beta \mathrm{OAc}$ & $\beta O A c$ & OAc & $\mathrm{OH}$ & $\beta \mathrm{OBz}$ & $\mathrm{OH}$ & $\mathrm{H}$ & A & {$[18,26]$} \\
\hline 20 & Wilforine & OAc & OAc & $\beta O A c$ & $\beta O A c$ & OAc & $\mathrm{OH}$ & $\beta \mathrm{OBz}$ & $\mathrm{H}$ & $\mathrm{H}$ & A & {$[18,26]$} \\
\hline 21 & Wilformine & OAc & OAc & $\beta \mathrm{OAc}$ & $\beta O A c$ & OAc & $\mathrm{OH}$ & $\beta \mathrm{OAc}$ & $\mathrm{H}$ & $\mathrm{H}$ & A & [18] \\
\hline 22 & Wilforidine & OAc & OAc & $\beta O A c$ & $\beta O A c$ & OAc & $\mathrm{OH}$ & $\beta \mathrm{OH}$ & $\mathrm{OH}$ & $\mathrm{H}$ & $\mathrm{A}$ & [18] \\
\hline 23 & Cangorinine E-1 & OAc & $\mathrm{OBz}$ & BOAc & BOAc & OAc & $\mathrm{OH}$ & $\beta O A c$ & $\mathrm{H}$ & $\mathrm{CH}_{3}$ & B & [18] \\
\hline 24 & Ebenifoline E-II & $\mathrm{OBz}$ & $\mathrm{OBz}$ & $\beta O A c$ & $\beta O A c$ & OAc & $\mathrm{OH}$ & $\beta \mathrm{OAc}$ & $\mathrm{H}$ & $\mathrm{CH}_{3}$ & B & [18] \\
\hline 25 & Neoeuonymine & OAc & $\mathrm{OH}$ & $\beta O A c$ & $\beta O A c$ & OAc & $\mathrm{OH}$ & $\beta O A c$ & $\mathrm{H}$ & $\mathrm{CH}_{3}$ & B & {$[18,24,26]$} \\
\hline 26 & Peritassine A & OAc & OAc & $\beta O A c$ & $\beta O A c$ & OAc & $\mathrm{OH}$ & $\beta O A c$ & $\mathrm{H}$ & $\mathrm{CH}_{3}$ & $\mathrm{C}$ & {$[18,21,31]$} \\
\hline 27 & Wilfornine G & OAc & OAc & $\beta \mathrm{ONic}$ & $\beta O A c$ & OAc & $\mathrm{OH}$ & $\beta O A c$ & $\mathrm{H}$ & $\mathrm{CH}_{3}$ & C & [18] \\
\hline 28 & Regelidine & $\mathrm{OBz}$ & ONic & $\mathrm{H}$ & $\alpha \mathrm{OBz}$ & $\mathrm{H}$ & $\mathrm{OH}$ & $\mathrm{H}$ & $\mathrm{H}$ & $\mathrm{H}$ & $\mathrm{D}$ & {$[18,24,28]$} \\
\hline 29 & $\begin{array}{l}\text { 9-O-trans-Cinnamoyl- } \\
\text { 9-debenzoylregelidine }\end{array}$ & $\mathrm{OBz}$ & ONic & $\mathrm{H}$ & $\alpha \mathrm{O} t \mathrm{Cin}$ & $\mathrm{H}$ & $\mathrm{OH}$ & $\mathrm{H}$ & $\mathrm{H}$ & $\mathrm{H}$ & $\mathrm{D}$ & {$[18]$} \\
\hline 30 & $\begin{array}{l}1 \beta \text {-Acetoxy- } 8 \alpha, 9 \beta \text {-dibenzoyloxy-13- } \\
\text { nicotinoyloxy- } \beta \text {-dihydroagarofuran }\end{array}$ & OAc & $\mathrm{H}$ & $\alpha \mathrm{OBz}$ & $\beta \mathrm{OBz}$ & ONic & $\mathrm{H}$ & $\mathrm{H}$ & $\mathrm{H}$ & $\mathrm{H}$ & $\mathrm{D}$ & [19] \\
\hline
\end{tabular}


Table 1. Cont

\begin{tabular}{|c|c|c|c|c|c|c|c|c|c|c|c|c|}
\hline No & Name & $\mathbf{R}_{\mathbf{1}}$ & $\mathbf{R}_{2}$ & $\mathbf{R}_{3}$ & $\mathbf{R}_{4}$ & $\mathbf{R}_{\mathbf{5}}$ & $\mathbf{R}_{6}$ & $\mathbf{R}_{7}$ & $\mathbf{R}_{8}$ & $\mathbf{R}_{\mathbf{9}}$ & Type & Ref \\
\hline 31 & $\begin{array}{l}1 \beta, 2 \beta \text {-Diacetoxy- } 9 \alpha \text {-benzoyloxy-13- } \\
\text { nicotinoyloxy- } \beta \text {-dihydroagarofuran }\end{array}$ & OAc & $\mathrm{H}$ & $\mathrm{H}$ & $\alpha \mathrm{OBz}$ & ONic & $\mathrm{H}$ & $\beta O A c$ & $\mathrm{H}$ & $\mathrm{H}$ & $\mathrm{D}$ & [19] \\
\hline 32 & Hypoglaunine E & OAc & $\mathrm{OH}$ & $\beta O A c$ & $\beta O A c$ & $\mathrm{OFu}$ & $\mathrm{OH}$ & $\beta O A c$ & $\mathrm{OH}$ & $\mathrm{CH}_{3}$ & $\mathrm{C}$ & {$[20,21,31]$} \\
\hline 33 & Hypoglaunine F & OAc & $\mathrm{OH}$ & $\beta O A c$ & $\beta O A c$ & OAc & $\mathrm{OH}$ & $\beta \mathrm{OFu}$ & $\mathrm{OH}$ & $\mathrm{CH}_{3}$ & $\mathrm{C}$ & {$[20,31]$} \\
\hline 34 & Triptersinine A & OtCin & $\mathrm{OH}$ & $\mathrm{O}$ & $\beta \mathrm{ONic}$ & OAc & $\mathrm{OH}$ & $\mathrm{H}$ & $\mathrm{H}$ & $\mathrm{H}$ & $\mathrm{D}$ & {$[21]$} \\
\hline 35 & Triptersinine B & OcCin & $\mathrm{OH}$ & $\mathrm{O}$ & $\beta \mathrm{ONic}$ & OAc & $\mathrm{OH}$ & $\mathrm{H}$ & $\mathrm{H}$ & $\mathrm{H}$ & $\mathrm{D}$ & [21] \\
\hline 36 & Triptersinine C & $\beta \mathrm{O} t \mathrm{Cin}$ & $\mathrm{OH}$ & $\beta \mathrm{OAc}$ & $\beta \mathrm{ONic}$ & OAc & $\mathrm{OH}$ & $\mathrm{H}$ & $\mathrm{H}$ & $\mathrm{H}$ & $\mathrm{D}$ & [21] \\
\hline 37 & Triptersinine D & OcCin & $\mathrm{OH}$ & $\beta O A c$ & $\beta \mathrm{ONic}$ & OAc & $\mathrm{OH}$ & $\mathrm{H}$ & $\mathrm{H}$ & $\mathrm{H}$ & $\mathrm{D}$ & [21] \\
\hline 38 & Triptersinine E & OcCin & OAc & $\beta O A c$ & $\beta \mathrm{ONic}$ & OAc & $\mathrm{OH}$ & $\mathrm{H}$ & $\mathrm{H}$ & $\mathrm{H}$ & $\mathrm{D}$ & [21] \\
\hline 39 & Triptersinine F & OAc & ONic & $\beta O A c$ & $\beta \mathrm{OFu}$ & OAc & $\mathrm{OH}$ & $\mathrm{H}$ & $\mathrm{H}$ & $\mathrm{H}$ & $\mathrm{D}$ & [21] \\
\hline 40 & Triptersinine G & OAc & OAc & $\beta \mathrm{ONic}$ & $\beta \mathrm{OFu}$ & OAc & $\mathrm{OH}$ & $\mathrm{H}$ & $\mathrm{H}$ & $\mathrm{H}$ & $\mathrm{D}$ & {$[21]$} \\
\hline 41 & Triptersinine $\mathrm{H}$ & $\mathrm{OFu}$ & OAc & $\beta \mathrm{ONic}$ & $\beta \mathrm{OFu}$ & OAc & $\mathrm{OH}$ & $\mathrm{H}$ & $\mathrm{H}$ & $\mathrm{H}$ & $\mathrm{D}$ & [21] \\
\hline 42 & Triptersinine L & OAc & ONic & $\beta \mathrm{OAc}$ & $\alpha \mathrm{OTig}$ & OAc & $\mathrm{OH}$ & $\mathrm{H}$ & $\mathrm{H}$ & $\mathrm{H}$ & $\mathrm{D}$ & [21] \\
\hline 43 & Wilfordinine A & OAc & OAc & $\beta O A c$ & $\beta O A c$ & OAc & $\mathrm{OH}$ & $\beta \mathrm{OH}$ & $\mathrm{H}$ & $\mathrm{CH}_{3}$ & $\mathrm{C}$ & {$[21,31]$} \\
\hline 44 & Hypoglaunine A & OAc & OAc & $\beta O A c$ & $\beta O A c$ & $\mathrm{OFu}$ & $\mathrm{OH}$ & $\beta O A c$ & $\mathrm{OH}$ & $\mathrm{CH}_{3}$ & $\mathrm{C}$ & {$[21,31]$} \\
\hline 45 & Wilfordinine E & OAc & OAc & $\beta O A c$ & $\beta O A c$ & OAc & $\mathrm{OH}$ & $\beta O A c$ & $\mathrm{H}$ & $\mathrm{H}$ & $\mathrm{F}$ & {$[21]$} \\
\hline 46 & Euonine & OAc & OAc & $\beta O A c$ & $\beta O A c$ & OAc & $\mathrm{OH}$ & $\beta O A c$ & $\mathrm{H}$ & $\mathrm{H}$ & $\mathrm{A}$ & {$[21,31]$} \\
\hline 47 & Evonine & OAc & OAc & $\mathrm{O}$ & $\alpha \mathrm{OAc}$ & OAc & $\mathrm{OH}$ & $\alpha \mathrm{OAc}$ & $\mathrm{H}$ & $\mathrm{CH}_{3}$ & $\mathrm{G}$ & {$[22]$} \\
\hline 48 & Neoevonine & OAc & $\mathrm{OH}$ & $\mathrm{O}$ & $\alpha \mathrm{OAc}$ & OAc & $\mathrm{OH}$ & $\alpha \mathrm{OAc}$ & $\mathrm{H}$ & $\mathrm{CH}_{3}$ & G & [22] \\
\hline 49 & $\begin{array}{c}1 \beta, 2 \beta, 5 \alpha, 8 \beta, 11 \text {-Pentaacetoxy- } \\
4 \alpha \text {-hydroxy-3 } \alpha \text {-(2-methylbutanoyl)- } \\
\text { 15-nicotinoyl-7-oxo-dihydroagarofuran }\end{array}$ & OAc & OAc & $\mathrm{O}$ & $\alpha \mathrm{OAc}$ & OAc & $\mathrm{OH}$ & $\alpha \mathrm{OAc}$ & $\mathrm{OMeBu}$ & ONic & $\mathrm{E}$ & [22] \\
\hline 50 & Triptersinine $\mathrm{M}$ & OtCin & OAc & $\beta \mathrm{OAc}$ & $\beta \mathrm{ONic}$ & OAc & $\mathrm{OH}$ & $\mathrm{H}$ & $\mathrm{H}$ & $\mathrm{H}$ & $\mathrm{D}$ & [23] \\
\hline 51 & Triptersinine $\mathrm{N}$ & ONic & $\mathrm{OFu}$ & $\beta O A c$ & $\beta \mathrm{OFu}$ & OAc & $\mathrm{OH}$ & $\mathrm{H}$ & $\mathrm{H}$ & $\mathrm{H}$ & $\mathrm{D}$ & [23] \\
\hline 52 & Triptersinine $\mathrm{O}$ & $\mathrm{OFu}$ & $\mathrm{OFu}$ & $\beta O A c$ & $\beta \mathrm{ONic}$ & OAc & $\mathrm{OH}$ & $\mathrm{H}$ & $\mathrm{H}$ & $\mathrm{H}$ & $\mathrm{D}$ & [23] \\
\hline 53 & Triptersinine $\mathrm{P}$ & OTig & OAc & $\beta \mathrm{ONic}$ & $\beta \mathrm{ONic}$ & OAc & $\mathrm{OH}$ & $\mathrm{H}$ & $\mathrm{H}$ & $\mathrm{H}$ & $\mathrm{D}$ & [23] \\
\hline 54 & Triptersinine $\mathrm{Q}$ & $\mathrm{OFu}$ & OAc & $\beta \mathrm{ONic}$ & $\beta$ OTig & OAc & $\mathrm{OH}$ & $\mathrm{H}$ & $\mathrm{H}$ & $\mathrm{H}$ & $\mathrm{D}$ & [23] \\
\hline 55 & Triptersinine R & OAc & OAc & $\beta \mathrm{ONic}$ & $\alpha \mathrm{OFu}$ & OAc & $\mathrm{OH}$ & $\mathrm{H}$ & $\mathrm{H}$ & $\mathrm{H}$ & $\mathrm{D}$ & [23] \\
\hline 56 & Triptersinine S & OAc & $\mathrm{OFu}$ & $\beta O A c$ & $\beta \mathrm{ONic}$ & OAc & $\mathrm{OH}$ & $\mathrm{H}$ & $\mathrm{H}$ & $\mathrm{H}$ & $\mathrm{D}$ & [23] \\
\hline 57 & Triptersinine $\mathrm{T}$ & OAc & $\mathrm{OH}$ & $\beta O A c$ & $\beta \mathrm{ONic}$ & OAc & $\mathrm{H}$ & $\mathrm{H}$ & $\mathrm{H}$ & $\mathrm{H}$ & $\mathrm{D}$ & [23] \\
\hline 58 & Tripterygiumine A & OAc & OAc & - & $\beta O A c$ & - & $\mathrm{OH}$ & $\beta \mathrm{OBz}$ & $\mathrm{H}$ & $\mathrm{CH}_{3}$ & $\mathrm{H}$ & [24] \\
\hline 59 & Tripterygiumine B & OAc & OAc & $\beta \mathrm{OBz}$ & $\beta O A c$ & OAc & $\mathrm{OH}$ & $\beta O A c$ & $\mathrm{H}$ & $\mathrm{CH}_{3}$ & $\mathrm{~B}$ & [24] \\
\hline 60 & Tripterygiumine C & OAc & $\mathrm{OBz}$ & $\beta O A c$ & $\beta O A c$ & OAc & $\mathrm{OH}$ & $\beta \mathrm{OBz}$ & $\mathrm{H}$ & $\mathrm{CH}_{3}$ & B & [24] \\
\hline 61 & Tripterygiumine D & $\mathrm{OH}$ & $\mathrm{OBz}$ & $\beta \mathrm{OH}$ & $\beta \mathrm{OH}$ & $\mathrm{OH}$ & $\mathrm{OH}$ & $\beta \mathrm{OH}$ & $\mathrm{H}$ & $\mathrm{CH}_{3}$ & B & {$[24]$} \\
\hline 62 & Tripterygiumine E & OAc & $\mathrm{OH}$ & $\beta O A c$ & $\beta O A c$ & OAc & $\mathrm{OH}$ & $\beta \mathrm{OFu}$ & $\mathrm{H}$ & $\mathrm{CH}_{3}$ & B & [24] \\
\hline
\end{tabular}


Table 1. Cont

\begin{tabular}{|c|c|c|c|c|c|c|c|c|c|c|c|c|}
\hline No & Name & $\mathbf{R}_{1}$ & $\mathbf{R}_{\mathbf{2}}$ & $\mathbf{R}_{\mathbf{3}}$ & $\mathbf{R}_{4}$ & $\mathbf{R}_{5}$ & $\mathbf{R}_{6}$ & $\mathbf{R}_{7}$ & $\mathbf{R}_{8}$ & $\mathbf{R}_{\mathbf{9}}$ & Type & Ref \\
\hline 63 & Tripterygiumine F & OAc & $\mathrm{OFu}$ & $\beta \mathrm{OAc}$ & $\beta O A c$ & OAc & $\mathrm{OH}$ & $\beta \mathrm{OBz}$ & $\mathrm{H}$ & $\mathrm{CH}_{3}$ & B & [24] \\
\hline 64 & Tripterygiumine G & OAc & $\mathrm{OBz}$ & $\beta \mathrm{OAc}$ & $\beta O A c$ & OAc & $\mathrm{OH}$ & $\beta \mathrm{OFu}$ & $\mathrm{H}$ & $\mathrm{CH}_{3}$ & B & [24] \\
\hline 65 & Tripterygiumine $\mathrm{H}$ & $\mathrm{OH}$ & OAc & $\beta \mathrm{OH}$ & $\beta \mathrm{OH}$ & $\mathrm{OH}$ & $\mathrm{OH}$ & $\beta \mathrm{OH}$ & $\mathrm{H}$ & $\mathrm{CH}_{3}$ & B & [24] \\
\hline 66 & Tripterygiumine I & OAc & $\mathrm{OH}$ & $\beta \mathrm{OAc}$ & $\beta O A c$ & OAc & $\mathrm{OH}$ & $\beta \mathrm{OBz}$ & $\mathrm{H}$ & $\mathrm{CH}_{3}$ & B & [24] \\
\hline 67 & Tripterygiumine J & OAc & $\mathrm{OH}$ & $\beta \mathrm{OH}$ & $\beta \mathrm{OAc}$ & OAc & $\mathrm{OH}$ & $\beta \mathrm{OAc}$ & $\mathrm{H}$ & $\mathrm{CH}_{3}$ & B & [24] \\
\hline 68 & Tripterygiumine $\mathrm{K}$ & OAc & $\mathrm{OH}$ & $\beta O A c$ & $\beta \mathrm{OAc}$ & $\mathrm{OBz}$ & $\mathrm{OH}$ & $\beta \mathrm{OH}$ & $\mathrm{H}$ & $\mathrm{CH}_{3}$ & $\mathrm{~B}$ & [24] \\
\hline 69 & Tripterygiumine L & ONic & $\mathrm{OH}$ & $\beta \mathrm{OAc}$ & $\beta O A c$ & OAc & $\mathrm{OH}$ & $\beta O A c$ & $\mathrm{H}$ & $\mathrm{CH}_{3}$ & B & [24] \\
\hline 70 & Hyponine D & OAc & $\mathrm{OBz}$ & $\beta \mathrm{OAc}$ & $\beta O A c$ & OAc & $\mathrm{OH}$ & $\beta \mathrm{ONic}$ & $\mathrm{H}$ & $\mathrm{CH}_{3}$ & B & [24] \\
\hline 71 & Hexadesacetyleuomynine & $\mathrm{OH}$ & $\mathrm{OH}$ & $\beta \mathrm{OH}$ & $\beta \mathrm{OH}$ & $\mathrm{OH}$ & $\mathrm{OH}$ & $\beta \mathrm{OH}$ & $\mathrm{H}$ & $\mathrm{CH}_{3}$ & B & {$[24]$} \\
\hline 72 & Euojaponine A & $\mathrm{OBz}$ & $\mathrm{OH}$ & $\beta \mathrm{OAc}$ & $\beta \mathrm{OAc}$ & OAc & $\mathrm{OH}$ & $\beta \mathrm{OAc}$ & $\mathrm{H}$ & $\mathrm{CH}_{3}$ & B & [24] \\
\hline 73 & Hyponine $\mathrm{C}$ & OAc & OAc & $\beta \mathrm{OAc}$ & $\beta O A c$ & $\mathrm{OBz}$ & $\mathrm{OH}$ & $\beta O A c$ & $\mathrm{H}$ & $\mathrm{CH}_{3}$ & B & [24] \\
\hline 74 & $\begin{array}{c}\text { 7-Acetyloxy-O }{ }^{11} \text {-benzoyl- } O^{2,11} \text { - deacetyl-7- } \\
\text { deoxoevonine }\end{array}$ & OAc & OAc & $\beta \mathrm{OAc}$ & $\beta O A c$ & $\mathrm{OBz}$ & $\mathrm{OH}$ & $\beta \mathrm{OH}$ & $\mathrm{H}$ & $\mathrm{CH}_{3}$ & B & {$[24]$} \\
\hline 75 & 4-Hydroxy-7-epi-chuchuhuanine E-V & OAc & OAc & BOAc & $\beta O A c$ & OAc & $\mathrm{OH}$ & $\beta \mathrm{OH}$ & $\mathrm{H}$ & $\mathrm{CH}_{3}$ & $\mathrm{~B}$ & {$[24,26]$} \\
\hline 76 & Wilfornine $\mathrm{F}$ & OAc & $\mathrm{OBz}$ & $\beta \mathrm{OAc}$ & $\beta O A c$ & OAc & $\mathrm{OH}$ & $\beta \mathrm{OH}$ & $\mathrm{H}$ & $\mathrm{CH}_{3}$ & B & [24] \\
\hline 77 & Tripterygiumine $\mathrm{M}$ & OAc & $\mathrm{OH}$ & $\mathrm{O}$ & $\beta O A c$ & OAc & $\mathrm{OH}$ & $\beta \mathrm{OBz}$ & $\mathrm{H}$ & $\mathrm{H}$ & A & [24] \\
\hline 78 & Tripterygiumine N & OAc & $\mathrm{OH}$ & $\mathrm{O}$ & $\beta O A c$ & OAc & $\mathrm{OH}$ & $\beta \mathrm{OBz}$ & $\mathrm{OFu}$ & $\mathrm{H}$ & $\mathrm{A}$ & [24] \\
\hline 79 & Tripterygiumine $\mathrm{O}$ & OAc & $\mathrm{OH}$ & $\beta O A c$ & $\beta O A c$ & OAc & $\mathrm{OH}$ & $\beta \mathrm{OFu}$ & $\mathrm{OBz}$ & $\mathrm{H}$ & A & [24] \\
\hline 80 & Tripterygiumine $\mathrm{P}$ & $\mathrm{OH}$ & OAc & $\beta \mathrm{OH}$ & $\beta \mathrm{OH}$ & $\mathrm{OH}$ & $\mathrm{OH}$ & $\beta \mathrm{OH}$ & $\mathrm{OBz}$ & $\mathrm{H}$ & A & [24] \\
\hline 81 & Tripterygiumine Q & $\mathrm{OH}$ & OAc & $\beta \mathrm{OH}$ & $\beta \mathrm{OH}$ & $\mathrm{OH}$ & $\mathrm{OH}$ & $\beta \mathrm{OH}$ & $\mathrm{OFu}$ & $\mathrm{H}$ & A & [24] \\
\hline 82 & Triptonine B & OAc & OAc & $\beta O A c$ & $\beta O A c$ & OAc & $\mathrm{OH}$ & $\beta \mathrm{OFu}$ & $\mathrm{OFu}$ & $\mathrm{H}$ & $\mathrm{A}$ & [24] \\
\hline 83 & 1-Desacetylwilforgine & $\mathrm{OH}$ & OAc & $\beta O A c$ & $\beta O A c$ & OAc & $\mathrm{OH}$ & $\beta \mathrm{OFu}$ & $\mathrm{H}$ & $\mathrm{H}$ & $\mathrm{A}$ & {$[24]$} \\
\hline 84 & Alatamine & OAc & OAc & $\mathrm{O}$ & $\beta O A c$ & OAc & $\mathrm{OH}$ & $\beta \mathrm{OBz}$ & $\mathrm{OH}$ & $\mathrm{H}$ & A & [24] \\
\hline 85 & Alatusinine & OAc & OAc & $\beta \mathrm{OAc}$ & $\beta \mathrm{OAc}$ & OAc & $\mathrm{OH}$ & $\beta O A c$ & $\mathrm{OH}$ & $\mathrm{H}$ & $\mathrm{A}$ & [24] \\
\hline 86 & Wilforzine & OAc & $\mathrm{OH}$ & $\beta O A c$ & $\beta O A c$ & OAc & $\mathrm{OH}$ & $\beta \mathrm{OBz}$ & $\mathrm{H}$ & $\mathrm{H}$ & $\mathrm{A}$ & [24] \\
\hline 87 & Wilforjine & OAc & OAc & $\beta \mathrm{OAc}$ & $\beta O A c$ & OAc & $\mathrm{OH}$ & $\beta \mathrm{OH}$ & $\mathrm{H}$ & $\mathrm{H}$ & A & {$[24,26]$} \\
\hline 88 & Tripterygiumine $\mathrm{R}$ & ONic & $\mathrm{OH}$ & $\mathrm{H}$ & $\alpha \mathrm{OBz}$ & $\mathrm{H}$ & $\mathrm{OH}$ & $\mathrm{H}$ & $\mathrm{H}$ & $\mathrm{H}$ & $\mathrm{D}$ & [24] \\
\hline 89 & $\begin{array}{c}\text { 1 } \beta, 5 \alpha, 11 \text {-Triacetoxy-7 } \beta- \\
\text { benzoyl- } 4 \alpha \text {-hydroxy- } 8 \beta- \\
\text { nicotinoyl-dihydroagarofuran }\end{array}$ & OAc & OAc & $\beta \mathrm{OBz}$ & $\alpha \mathrm{ONic}$ & OAc & $\mathrm{OH}$ & $\mathrm{H}$ & $\mathrm{H}$ & $\mathrm{H}$ & $\mathrm{D}$ & [24] \\
\hline 90 & Wilforcidine & $\mathrm{OBz}$ & ONic & $\mathrm{H}$ & $\alpha \mathrm{OtCin}$ & $\mathrm{H}$ & $\mathrm{OH}$ & $\mathrm{H}$ & $\mathrm{H}$ & $\mathrm{H}$ & $\mathrm{D}$ & {$[24]$} \\
\hline 91 & $\begin{array}{l}5 \alpha \text {-Benzoyl- } 4 \alpha \text {-hydroxy- } 1 \beta, 8 \alpha- \\
\text { dinicotinoyl-dihydroagarofuran }\end{array}$ & ONic & $\mathrm{OBz}$ & $\mathrm{H}$ & $\alpha \mathrm{ONic}$ & $\mathrm{H}$ & $\mathrm{OH}$ & $\mathrm{H}$ & $\mathrm{H}$ & $\mathrm{H}$ & $\mathrm{D}$ & [24] \\
\hline
\end{tabular}


Table 1. Cont

\begin{tabular}{|c|c|c|c|c|c|c|c|c|c|c|c|c|}
\hline No & Name & $\mathbf{R}_{1}$ & $\mathbf{R}_{2}$ & $\mathbf{R}_{3}$ & $\mathbf{R}_{4}$ & $\mathbf{R}_{\mathbf{5}}$ & $\mathbf{R}_{6}$ & $\mathbf{R}_{7}$ & $\mathbf{R}_{8}$ & $\mathbf{R}_{9}$ & Type & Ref \\
\hline 92 & $\begin{array}{c}1 \alpha, 2 \alpha, 6 \beta, 8 \beta, 9 \alpha, 15-\text { Hexacetoxy- } \\
4 \beta \text {-hydroxy- } 3 \beta, 13-[2 \text { - } \\
\text { (3-carboxybutyl)]nicotinic } \\
\text { acid-dicarbolactone- } \beta \text {-di hydroagarofuran }\end{array}$ & OAc & OAc & $\beta O A c$ & $\alpha \mathrm{OAc}$ & OAc & $\mathrm{OH}$ & $\alpha \mathrm{OAc}$ & $\mathrm{H}$ & $\mathrm{H}$ & I & [25] \\
\hline 93 & $\begin{array}{c}1 \alpha, 2 \alpha, 9 \alpha, 15 \text {-Tetracetoxy- } 4 \beta, 6 \beta \text {-dihydroxy- } \\
\text { 8-oxo,3 } 3,13 \text { - }\left[4^{\prime} \text { - }\right. \\
\text { (3-carboxybutyl)]nicotinicacid- } \\
\text { dicarbolactone- } \beta \text {-dihydroagarofuran }\end{array}$ & OAc & $\mathrm{OH}$ & $\mathrm{O}$ & $\beta O A c$ & OAc & $\mathrm{OH}$ & $\alpha \mathrm{OAc}$ & $\mathrm{H}$ & $\mathrm{H}$ & $\mathrm{J}$ & [25] \\
\hline 94 & $\begin{array}{c}1 \alpha, 2 \alpha, 9 \alpha, 15 \text {-Tetracetoxy- } \\
4 \beta, 6 \beta, 8 \beta \text {-trihydroxy- } 3 \beta, 13-\left[4^{\prime}-\right. \\
\text { (3-carboxybutyl)]nicotinic acid- } \\
\text { dicarbolactone- } \beta \text {-dihydroagarofuran }\end{array}$ & OAc & $\mathrm{OH}$ & $\beta \mathrm{OH}$ & $\beta O A c$ & OAc & $\mathrm{OH}$ & $\alpha \mathrm{OAc}$ & $\mathrm{H}$ & $\mathrm{H}$ & $\mathrm{J}$ & [25] \\
\hline 95 & $\begin{array}{c}1 \alpha, 2 \alpha, 8 \beta, 9 \alpha, 15 \text {-Pentacetoxy- } \\
4 \beta, 6 \beta \text {-dihydroxy- } 3 \beta, 13- \\
{\left[4^{\prime}-(3-\text {-carboxybutyl)]nicotinic }\right.} \\
\text { acid-dicarbolactone- } \beta \text { - dihydroagarofuran }\end{array}$ & OAc & $\mathrm{OH}$ & $\beta O A c$ & $\beta O A c$ & OAc & $\mathrm{OH}$ & $\alpha \mathrm{OAc}$ & $\mathrm{H}$ & $\mathrm{H}$ & $\mathrm{J}$ & [25] \\
\hline 96 & Tripterygiumine $S$ & $\mathrm{OAc}$ & OAc & $\mathrm{O}$ & $\beta O A c$ & OAc & $\mathrm{OH}$ & $\beta \mathrm{OH}$ & $\mathrm{OFu}$ & $\mathrm{H}$ & A & [26] \\
\hline 97 & Tripterygiumine $\mathrm{T}$ & OAc & $\mathrm{OH}$ & $\mathrm{O}$ & $\beta \mathrm{OAc}$ & OAc & $\mathrm{OH}$ & $\beta \mathrm{OH}$ & $\mathrm{OH}$ & $\mathrm{H}$ & A & [26] \\
\hline 98 & Tripterygiumine U & OAc & OAc & $\mathrm{O}$ & $\beta O A c$ & OAc & $\mathrm{OH}$ & $\beta \mathrm{OH}$ & $\mathrm{H}$ & $\mathrm{H}$ & $\mathrm{A}$ & [26] \\
\hline 99 & Tripterygiumine V & OAc & OAc & $\beta \mathrm{OAc}$ & $\beta \mathrm{OAc}$ & OAc & $\mathrm{OH}$ & $\beta \mathrm{OH}$ & $\mathrm{OBz}$ & $\mathrm{H}$ & A & [26] \\
\hline 100 & Tripterygiumine $\mathrm{W}$ & $\mathrm{OFu}$ & $\mathrm{OBz}$ & $\beta O A c$ & $\beta O A c$ & OAc & $\mathrm{OH}$ & $\beta \mathrm{OH}$ & $\mathrm{H}$ & $\mathrm{CH}_{3}$ & $\mathrm{~B}$ & [26] \\
\hline 101 & Wilfornine A & OAc & OAc & $\beta O A c$ & $\beta O A c$ & OAc & $\mathrm{OH}$ & $\beta O A c$ & $\mathrm{OBz}$ & $\mathrm{H}$ & A & [26] \\
\hline 102 & Wilfornine D & $\mathrm{OAc}$ & OAc & $\beta O A c$ & $\beta O A c$ & OAc & $\mathrm{OH}$ & $\beta O A c$ & $\mathrm{OFu}$ & $\mathrm{H}$ & A & [26] \\
\hline 103 & Tripfordine A & OAc & OAc & $\beta O A c$ & $\beta O A c$ & OAc & $\mathrm{OH}$ & $\beta \mathrm{OH}$ & $\mathrm{OH}$ & $\mathrm{H}$ & A & [26] \\
\hline 104 & $\begin{array}{l}\text { 2-Debenzoyl-2-nicotinoylwilforine } \\
\text { (+)-(1R,2S,4S,5S,6R,7R,9S,10R)- }\end{array}$ & OAc & OAc & $\beta \mathrm{OAc}$ & $\beta O A c$ & OAc & $\mathrm{OH}$ & $\beta \mathrm{ONic}$ & $\mathrm{H}$ & $\mathrm{H}$ & A & {$[26]$} \\
\hline 105 & $\begin{array}{l}\text { 1,2,15-Triacetoxy-9-benzoyloxy- } 6 \text { - } \\
\text { nicotinoyloxydihydro- } \beta \text {-agarofuran }\end{array}$ & OAc & ONic & $\mathrm{H}$ & $\beta \mathrm{OBz}$ & OAc & $\mathrm{OH}$ & $\alpha \mathrm{OAc}$ & $\mathrm{H}$ & $\mathrm{H}$ & $\mathrm{E}$ & [27] \\
\hline 106 & Triptregeline A & ONic & $\mathrm{OH}$ & $\beta O A c$ & $\alpha \mathrm{OBz}$ & OAc & $\mathrm{OH}$ & $\alpha \mathrm{OAc}$ & $\mathrm{H}$ & $\mathrm{H}$ & E & [28] \\
\hline 107 & Triptregeline B & ONic & OAc & $\alpha \mathrm{OAc}$ & $\alpha \mathrm{OBz}$ & OAc & $\mathrm{OH}$ & $\mathrm{H}$ & $\mathrm{H}$ & $\mathrm{H}$ & $\mathrm{E}$ & [28] \\
\hline 108 & Triptregeline C & ONic & OAc & $\alpha \mathrm{OH}$ & $\alpha \mathrm{OBz}$ & $\mathrm{OH}$ & $\mathrm{OH}$ & $\mathrm{H}$ & $\mathrm{H}$ & $\mathrm{H}$ & $\mathrm{E}$ & [28] \\
\hline 109 & Triptregeline D & $\mathrm{OFu}$ & OAc & $\alpha \mathrm{ONic}$ & $\alpha \mathrm{OBz}$ & OAc & $\mathrm{OH}$ & $\mathrm{H}$ & $\mathrm{H}$ & $\mathrm{H}$ & $\mathrm{E}$ & [28] \\
\hline 110 & Triptregeline E & $\mathrm{OFu}$ & $\mathrm{OH}$ & $\alpha \mathrm{ONic}$ & $\alpha \mathrm{OBz}$ & OAc & $\mathrm{OH}$ & $\mathrm{H}$ & $\mathrm{H}$ & $\mathrm{H}$ & E & [28] \\
\hline 111 & Triptregeline F & OAc & $\mathrm{OH}$ & $\alpha \mathrm{ONic}$ & $\alpha \mathrm{OBz}$ & OAc & $\mathrm{OH}$ & $\mathrm{H}$ & $\mathrm{H}$ & $\mathrm{H}$ & $\mathrm{E}$ & [28] \\
\hline 112 & Triptregeline G & $\mathrm{OFu}$ & $\mathrm{OH}$ & $\alpha \mathrm{ONic}$ & $\alpha \mathrm{OAc}$ & OAc & $\mathrm{OH}$ & $\mathrm{H}$ & $\mathrm{H}$ & $\mathrm{H}$ & $\mathrm{E}$ & [28] \\
\hline
\end{tabular}


Table 1. Cont

\begin{tabular}{|c|c|c|c|c|c|c|c|c|c|c|c|c|}
\hline No & Name & $\mathbf{R}_{1}$ & $\mathbf{R}_{\mathbf{2}}$ & $\mathbf{R}_{\mathbf{3}}$ & $\mathbf{R}_{\mathbf{4}}$ & $\mathbf{R}_{5}$ & $\mathbf{R}_{6}$ & $\mathbf{R}_{7}$ & $\mathbf{R}_{8}$ & $\mathbf{R}_{\mathbf{9}}$ & Type & Ref \\
\hline 113 & Triptregeline $\mathrm{H}$ & $\mathrm{OBz}$ & OAc & $\alpha \mathrm{OH}$ & $\alpha \mathrm{ONic}$ & OAc & $\mathrm{OH}$ & $\mathrm{H}$ & $\mathrm{H}$ & $\mathrm{H}$ & E & [28] \\
\hline 114 & Triptregeline I & $\mathrm{OFu}$ & ONic & $\mathrm{H}$ & $\beta \mathrm{OBz}$ & $\mathrm{H}$ & $\mathrm{OH}$ & $\beta O A c$ & $\mathrm{H}$ & $\mathrm{H}$ & $\mathrm{E}$ & [28] \\
\hline 115 & Triptregeline J & $\mathrm{OBz}$ & ONic & $\mathrm{H}$ & $\beta \mathrm{OBz}$ & $\mathrm{H}$ & $\mathrm{OH}$ & $\mathrm{H}$ & $\mathrm{H}$ & $\mathrm{H}$ & $\mathrm{E}$ & [28] \\
\hline 116 & $\begin{array}{c}1 \alpha, 6 \beta, 15 \text {-Triacetoxy- } 8 \alpha \text {-benzoyloxy- } \\
4 \beta \text {-hydroxyl }-9 \alpha \text {-(3-nicotinoyloxy)- } \\
\text { dihydro- } \beta \text {-agarofuran }\end{array}$ & OAc & OAc & $\alpha \mathrm{OBz}$ & $\alpha \mathrm{ONic}$ & OAc & $\mathrm{OH}$ & $\mathrm{H}$ & $\mathrm{H}$ & $\mathrm{H}$ & $\mathrm{E}$ & [28] \\
\hline 117 & Dimacroregeline A & $\mathrm{OH}$ & OAc & $\mathrm{H}$ & $\alpha \mathrm{OH}$ & - & $\mathrm{OH}$ & $\alpha \mathrm{OH}$ & $\mathrm{H}$ & $\mathrm{CH}_{3}$ & K & [29] \\
\hline 118 & Dimacroregeline B & $\mathrm{OH}$ & OAc & OAc & $\alpha \mathrm{OH}$ & - & $\mathrm{OH}$ & $\alpha \mathrm{OH}$ & $\mathrm{H}$ & $\mathrm{CH}_{3}$ & K & [29] \\
\hline 119 & Triptonine A & OAc & OAc & - & $\alpha \mathrm{OAc}$ & - & $\mathrm{OH}$ & $\alpha \mathrm{OAc}$ & $\mathrm{H}$ & $\mathrm{CH}_{3}$ & $\mathrm{~L}$ & [29] \\
\hline 120 & 4-Deoxyalatamine & OAc & OAc & $\mathrm{O}$ & $\alpha \mathrm{OAc}$ & OAc & $\mathrm{H}$ & $\alpha \mathrm{OAc}$ & $\mathrm{OH}$ & $\mathrm{H}$ & I & [30] \\
\hline 121 & 1-O-Benzoyl-1-deacetyl-4-deoxyalatamine & $\mathrm{OBz}$ & OAc & $\mathrm{O}$ & $\alpha \mathrm{OAc}$ & OAc & $\mathrm{H}$ & $\alpha \mathrm{OAc}$ & $\mathrm{OH}$ & $\mathrm{H}$ & I & [30] \\
\hline 122 & $\begin{array}{c}\text { 1, 2-O-Dibenzoyl-1, } \\
\text { 2-deacetyl-4-deoxyalatamine }\end{array}$ & $\mathrm{OBz}$ & OAc & $\mathrm{O}$ & $\alpha \mathrm{OAc}$ & OAc & $\mathrm{H}$ & $\alpha \mathrm{OBz}$ & $\mathrm{OH}$ & $\mathrm{H}$ & I & [30] \\
\hline 123 & 4-Deoxyisowilfordine & OAc & OAc & $\beta \mathrm{OAc}$ & $\alpha \mathrm{OAc}$ & OAc & $\mathrm{H}$ & $\alpha \mathrm{OBz}$ & $\mathrm{OH}$ & $\mathrm{H}$ & $\mathrm{J}$ & {$[30]$} \\
\hline 124 & Triptersinine U & OAc & OAc & $\beta \mathrm{OAc}$ & $\beta O A c$ & OAc & $\mathrm{OH}$ & $\beta O A c$ & $\alpha \mathrm{ONic}$ & ONic & $\mathrm{D}$ & [31] \\
\hline 125 & Hypoglaunine B & OAc & OAc & $\beta \mathrm{OAc}$ & $\beta \mathrm{OAc}$ & $\mathrm{OFu}$ & $\mathrm{OH}$ & $\beta \mathrm{OAc}$ & $\mathrm{OH}$ & $\mathrm{CH}_{3}$ & C & [31] \\
\hline 126 & Triptersinine Z4 & $\mathrm{OFu}$ & OAc & $\beta O A c$ & $\beta \mathrm{ONic}$ & OAc & $\mathrm{H}$ & $\mathrm{H}$ & $\mathrm{H}$ & $\mathrm{H}$ & $\mathrm{D}$ & [32] \\
\hline 127 & Triptersinine Z5 & OAc & $\mathrm{OFu}$ & $\beta \mathrm{OAc}$ & $\beta \mathrm{ONic}$ & OAc & $\mathrm{H}$ & $\mathrm{H}$ & $\mathrm{H}$ & $\mathrm{H}$ & $\mathrm{D}$ & [32] \\
\hline 128 & Triptersinine Z6 & $\mathrm{OFu}$ & $\mathrm{OFu}$ & $\beta \mathrm{OAc}$ & $\beta \mathrm{ONic}$ & OAc & $\mathrm{H}$ & $\mathrm{H}$ & $\mathrm{H}$ & $\mathrm{H}$ & $\mathrm{D}$ & [32] \\
\hline 129 & Triptersinine Z7 & OcCin & OAc & $\beta O A c$ & $\beta \mathrm{ONic}$ & OAc & $\mathrm{H}$ & $\mathrm{H}$ & $\mathrm{H}$ & $\mathrm{H}$ & $\mathrm{D}$ & [32] \\
\hline 130 & Triptersinine Z8 & OtCin & OAc & $\beta \mathrm{OAc}$ & $\beta \mathrm{ONic}$ & OAc & $\mathrm{H}$ & $\mathrm{H}$ & $\mathrm{H}$ & $\mathrm{H}$ & $\mathrm{D}$ & [32] \\
\hline 131 & Euojaponine C & $\mathrm{OBz}$ & $\mathrm{OBz}$ & $\beta \mathrm{OAc}$ & $\beta \mathrm{OAc}$ & OAc & $\mathrm{OH}$ & $\beta \mathrm{OH}$ & $\mathrm{H}$ & $\mathrm{CH}_{3}$ & B & [32] \\
\hline 132 & Triptersinine Z9 & OcCin & $\mathrm{OFu}$ & $\beta \mathrm{OAc}$ & $\beta \mathrm{ONic}$ & OAc & $\mathrm{OH}$ & $\mathrm{H}$ & $\mathrm{H}$ & $\mathrm{H}$ & $\mathrm{D}$ & [33] \\
\hline 133 & Triptersinine Z10 & OtCin & $\mathrm{OFu}$ & $\beta O A c$ & $\beta \mathrm{ONic}$ & OAc & $\mathrm{OH}$ & $\mathrm{H}$ & $\mathrm{H}$ & $\mathrm{H}$ & $\mathrm{D}$ & [33] \\
\hline 134 & Triptersinine Z11 & OtCin & OAc & $\beta \mathrm{ONic}$ & $\beta \mathrm{OFu}$ & OAc & $\mathrm{OH}$ & $\mathrm{H}$ & $\mathrm{H}$ & $\mathrm{H}$ & $\mathrm{D}$ & [33] \\
\hline 135 & Triptersinine Z12 & OcCin & OAc & $\beta O N i c$ & $\beta \mathrm{OFu}$ & OAc & $\mathrm{OH}$ & $\mathrm{H}$ & $\mathrm{H}$ & $\mathrm{H}$ & $\mathrm{D}$ & [33] \\
\hline 136 & Triptersinine Z13 & ONic & $\mathrm{OFu}$ & $\beta O A c$ & $\beta$ OTig & OAc & $\mathrm{OH}$ & $\mathrm{H}$ & $\mathrm{H}$ & $\mathrm{H}$ & $\mathrm{D}$ & [33] \\
\hline 137 & Triptersinine Z14 & OAc & $\mathrm{OFu}$ & $\beta \mathrm{ONic}$ & $\beta$ OTig & OAc & $\mathrm{OH}$ & $\mathrm{H}$ & $\mathrm{H}$ & $\mathrm{H}$ & $\mathrm{D}$ & {$[33]$} \\
\hline 138 & Chinese bittersweet alkaloid A & OAc & OAc & $\beta \mathrm{OAc}$ & $\beta O A c$ & $\mathrm{OiBu}$ & $\mathrm{OH}$ & $\beta \mathrm{OH}$ & $\mathrm{H}$ & $\mathrm{CH}_{3}$ & $\mathrm{~B}$ & [34] \\
\hline 139 & Chinese bittersweet alkaloid B & OAc & OAc & $\beta O A c$ & $\beta O A c$ & $\mathrm{OiBu}$ & $\mathrm{OH}$ & $\beta O A c$ & $\mathrm{H}$ & $\mathrm{CH}_{3}$ & B & [34] \\
\hline 140 & Monimin I & ONic & ONic & $\mathrm{H}$ & $\alpha \mathrm{OAc}$ & $\mathrm{H}$ & $\mathrm{H}$ & $\mathrm{H}$ & $\mathrm{H}$ & $\mathrm{H}$ & E & [35] \\
\hline 141 & Monimin II & ONic & ONic & $\alpha \mathrm{OH}$ & $\alpha \mathrm{OBz}$ & $\mathrm{H}$ & $\mathrm{H}$ & $\mathrm{H}$ & $\mathrm{H}$ & $\mathrm{H}$ & E & [35] \\
\hline 142 & Tripteryford C & ONic & $\mathrm{OH}$ & $\beta O A c$ & $\alpha \mathrm{OAc}$ & OAc & $\mathrm{H}$ & $\alpha \mathrm{OAc}$ & $\beta \mathrm{OH}$ & $\mathrm{H}$ & E & [36] \\
\hline 143 & Tripteryford E & ONic & OAc & $\alpha \mathrm{OH}$ & $\beta \mathrm{OFu}$ & OAc & $\mathrm{OH}$ & $\alpha \mathrm{OAc}$ & $\beta \mathrm{OH}$ & $\mathrm{H}$ & $\mathrm{E}$ & [36] \\
\hline 144 & Celaspaculin G & OAc & $\mathrm{OBz}$ & $\beta O A c$ & $\alpha \mathrm{ONic}$ & $\mathrm{H}$ & $\mathrm{OH}$ & $\mathrm{H}$ & $\mathrm{H}$ & $\mathrm{H}$ & $\mathrm{E}$ & [37] \\
\hline
\end{tabular}




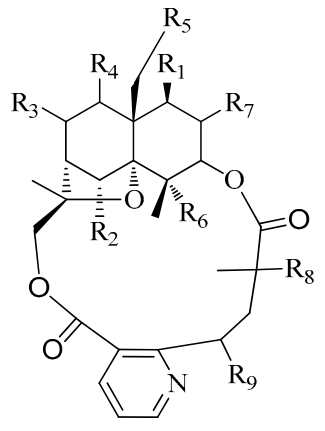

A

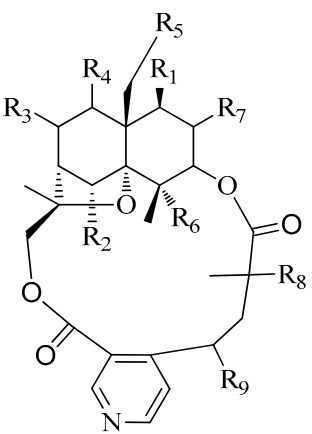

F

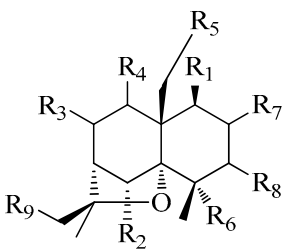

D

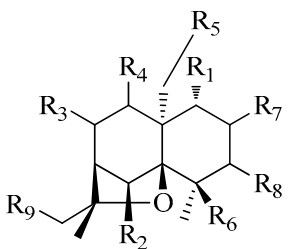

E

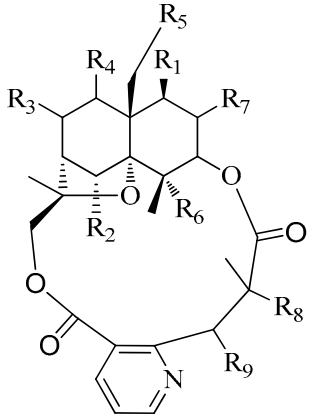

B

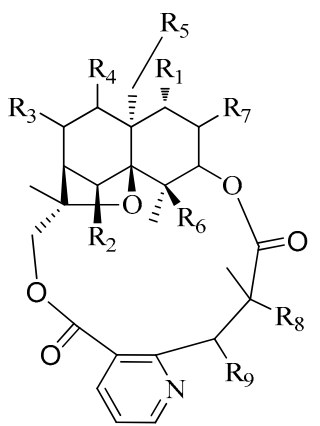

G

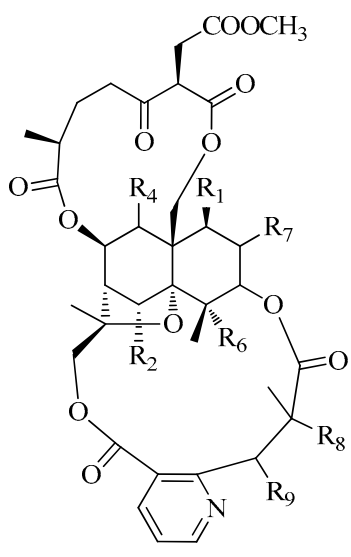

H

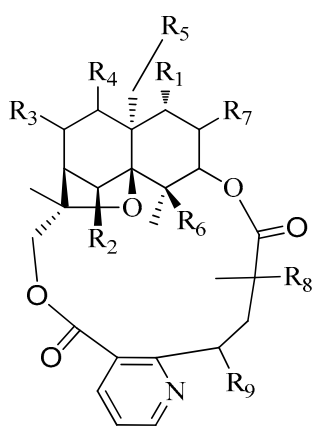

I

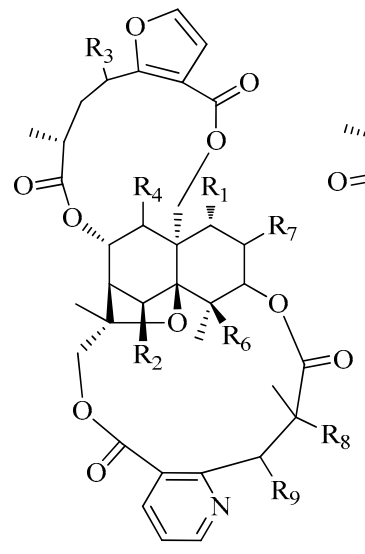

K

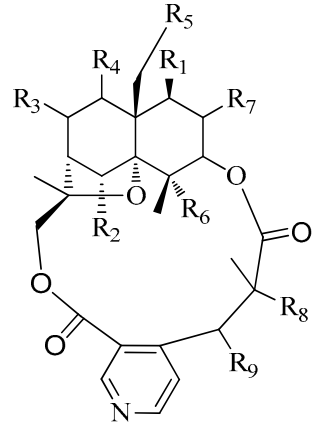

C

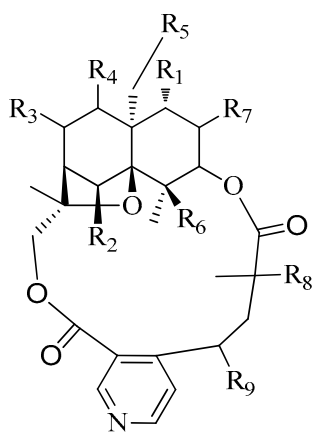

J

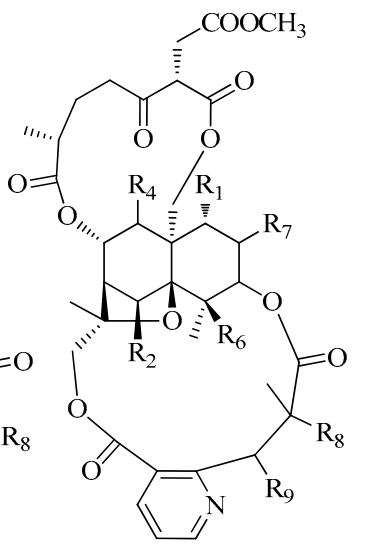

L

Figure 1. Twelve types (A-L) of dihydroagarofuran sesquiterpenoid skeletons.

\subsection{Drimane and Friedo-Drimane Sesquiterpenoids}

Nitrobenzoyl drimane sesquiterpenoids are rare in natural sources, Aspergillus fungi species being the only known sources.6 $\beta, 9 \alpha$-Dihydroxy-14- $p$-nitrobenzoylcinnamolide (145) and insulicolide A (146), insulicolide B (147), 14-O-acetylinsulicolide A (148), insulicolide C (149) and 9-deoxyinsulicolide A (150) (Figure 2) were isolated from extracts of the culture of marine-derived fungus Aspergillus ochraceus Jcma1F17 [38,39]. All of them displayed significant cytotoxicity against 10 human cancer celllines (H1975, U937, K562, BGC-823, Molt-4, MCF-7, A549, Hela, HL60, and Huh-7), with IC 50 values ranging from $1.95 \mathrm{mM}$ to $6.35 \mathrm{mM}$, and 145 also exhibited moderate inhibitory activity against two viruses, $\mathrm{H} 3 \mathrm{~N} 2$ and EV71, with $\mathrm{IC}_{50}$ values of 17.0 and $9.4 \mathrm{mM}$, respectively [38]. Compound 146 showed the strongest activities, with $\mathrm{IC}_{50}$ values of $1.5,1.5$, and $0.89 \mu \mathrm{M}$, against ACHN, OSRC-2, and 786-O cells, respectively [39]. 148 indicated potent inhibitory activities at low $\mu \mathrm{M}$ levels, comparable to the positive control, sorafenib, adrug (Nexavar) approved for the treatment of primary kidneycancer 
(advanced renal cell carcinoma) [39]. Additionally, 145 and 148 exhibited stronger cytotoxicity to 786-O cells ( $\mathrm{IC}_{50} 4.3$ and $2.3 \mu \mathrm{M}$, respectively) than to OS-RC-2 ( $\mathrm{IC}_{50} 8.2$ and $5.3 \mu \mathrm{M}$, respectively) and $\mathrm{ACHN}$ ( $\mathrm{IC}_{50} 11$ and $4.1 \mu \mathrm{M}$, respectively) [39]. Purpuride (151), berkedrimane B (152), minioluteumides A-D (153, 154, 156 and 157), purpuride B (155) (Figure 2) featuring with lactones conjugated a $\mathrm{N}$-acetyl-L-valine, and such drimane sesquiterpenoid are rare in nature, which were extracted from the marine fungus, Talaromyces minioluteus (Penicillium minioluteum) [40]. Compounds 152, 153 and 157 exhibited cytotoxic activity with $\mathrm{IC}_{50}$ values of 193.3, 50.6 and $57.0 \mu \mathrm{M}$ against HepG2 cancer cell line, respectively [40]. A new sesquiterpene lactonepurpuride D (158), berkedrimane A (159), along with 151, 152, 155, 157 (Figure 2) were prepared from a culture of marine-sourced fungus Penicillum ZZ1283 in the medium of potato dextrose broth was found to have antimicrobial activities with MIC values of 4-14 $\mu \mathrm{g} / \mathrm{mL}$ against MRSA [41]. Saccharoquinoline (160) (Figure 2) composing of a drimane-type sesquiterpene unit in combination with anapparent 6,7,8-trihydroxyquinoline-2-carboxylic acid with cytotoxicity against the HCT-116 cancer cell line by inducing G1 arrest, and was obtained from the fermentation broth of the marine-derived bacterium Saccharomonospora sp. CNQ-490 [42].

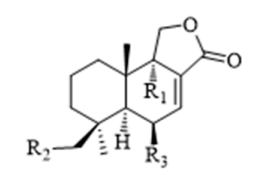

$145 \mathrm{R}_{1}=\mathrm{OH} ; \mathrm{R}_{2}=\mathrm{OPN} ; \mathrm{R}_{3}=\mathrm{OH}$ $146 \mathrm{R}_{1}=\mathrm{OH} ; \mathrm{R}_{2}=\mathrm{OH} ; \mathrm{R}_{3}=\mathrm{OPN}$ $147 \mathrm{R}_{1}=\mathrm{H} ; \mathrm{R}_{2}=\mathrm{OPN} ; \mathrm{R}_{3}=\mathrm{OH}$ $148 \mathrm{R}_{1}=\mathrm{OH} ; \mathrm{R}_{2}=\mathrm{OAc} ; \mathrm{R}_{3}=\mathrm{OPN}$ $149 \mathrm{R}_{1}=\mathrm{OH} ; \mathrm{R}_{2}=\mathrm{OPN} ; \mathrm{R}_{3}=\mathrm{OH}$ $150 \mathrm{R}_{1}=\mathrm{H} ; \mathrm{R}_{2}=\mathrm{OH} ; \mathrm{R}_{3}=\mathrm{OPN}$
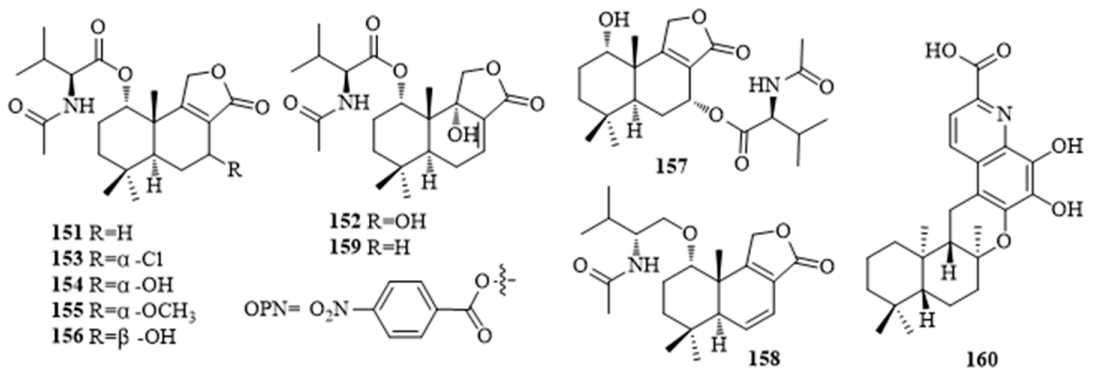

Figure 2. The structures of compounds 145-160.

Marine sponges are a rich source of bioactive secondary metabolites, the majority of which are sesquiterpene quinones/hydroquinones, most of which possess either adrimane or a rearranged 4,9-friedodrimane terpenoid skeleton, which contains a C15 sesquiterpene moiety incorporating a C6 benzoquinone or hydroquinone group framework. Drimane sesquiterpene quinones represent a large group of biologically active marine natural products. Six nitrogenous drimane sesquiterpenoid aminoquinones (Figure 3 and Table 2), named 18-aminoarenarone (161), 19-aminoarenarone (162), 18-methylaminoarenarone (163), 19-methylaminoarenarone (164), along with two dimeric popolohuanone F (165), popolohuanone A (166) isolated from the Australian marine sponge Dysidea sp., and 165 and 166 showed DPPH radical scavenging activity with $\mathrm{IC}_{50}$ values of 35.0 and $35.0 \mu \mathrm{M}$, respectively [43]. A new sesquiterpene benzoxazole, nakijinol B (167), its acetylated derivative, nakijinol B diacetate (170), and two newsesquiterpene quinones, smenospongines B (168) and C (169) (Figure 3 and Table 2), were extracted from the methanol extract of the marine sponge Dactylospongia elegans, and were found to have cytotoxic activities in the range of 1.8-46 $\mu \mathrm{M}$ against a panel of human tumor cell lines (SF-268, H460, MCF-7, and HT-29) and a normal mammalian cell line (CHO-K1) [44]. Investigation of the marine sponge Dysideaavara, three bioactive sesquiterpenoid Quinones afforded, (-)-3'-methylaminoavarone (171), (-)-4'-methylaminoavarone (172) and (-)-N-methylmelemeleone-A (173) (Figure 3 and Table 2) with their moderate protein kinase inhibition, cytotoxicity, inhibition of NFkB-activity and insecticidal activity [45]. Two sesquiterpene aminoquinines (Figure 3 and Table 2), smenospongine (174) and glycinylilimaquinone (175), were isolated from the Fijian marine sponge Hippospongia sp., and displayed lethality at $\mathrm{LD}_{50}=188$ and $<500$ ppm against brineshrimp, respectively [46]. Bioactivity-guided isolation yielded five new sesquiterpene aminoquinones 5-epi-Nakijiquinone S-N (176-180), two new sesquiterpene benzoxazoles 5-epi-Nakijinol C-D (181 and 182) (Figure 3 and Table 2) isolated from the sponge Dactylospongia metachromia [47]. Compounds 176-180 showed potent cytotoxicity againstthe mouse lymphoma cell line $\mathrm{L} 5178 Y$ with $\mathrm{IC}_{50}$ values ranging from 1.1 to $3.7 \mu \mathrm{M}$ [47]. When tested in vitro 
for their inhibitory potential against 16 different protein kinases, compounds $\mathbf{1 8 0}$ and $\mathbf{1 8 1}$ exhibited the strongest inhibitory activity against ALK, FAK, IGF1-R, SRC, VEGF-R2, Aurora-B, MET wt, and NEK6 kinases ( $\mathrm{IC}_{50}$ 0.97-8.62 $\mu \mathrm{M}$ ) [47]. Dysidaminones A-M (183-195) (Figure 3 and Table 2), thirteen new sesquiterpene aminoquinones, along with six known ones (196-201), were isolated from the South China Sea sponge Dysidea fragilis [48]. Compounds 185, 187, 190, and 192, 196, and 198 showed cytotoxicity against mouse B16F10 melanoma and human NCI-H929 myeloma, HepG2 hepatoma, and SK-OV-3 ovarian cancer cell lines [48]. In addition, these six cytotoxic compounds also exhibited NF-kB inhibitory activity with $\mathrm{IC}_{50}$ values of $0.05-0.27 \mathrm{mM}$ [48]. Four nitrogenous 4,9-friedodrimane-type sesquiterpenoids (202-205) (Figure 3 and Table 2) were acquired using the oxidative potential of Verongula rigida on bioactive metabolites from two Smenospongia sponges, and the mixture of 204 and 205 suppressed $\beta$-cateninresponse transcription (CRT) via degrading $\beta$-catenin and exhibited cytotoxic activity on colon cancer cells [49]. Compounds 206-214, together with 174 (Figure 3 and Table 2) have been obtained from the Marine Sponge Spongiapertusa Esper, and 174, 213, 214 exhibited activities against the human cancer cell lines U937, HeLa, and HepG2, with most potent cytotoxicities to U937 cells with $\mathrm{IC}_{50}$ values of1.5, 2.8, and $0.6 \mu \mathrm{M}$, respectively [50]. Four sesquiterpene hydroquinones, dactylospongins A-D (215-218), as wellas five sesquiterpene quinones, melemeleones B-E (219-222) and dysidaminone N (223) (Figure 3 and Table 2) were isolated from the marine sponge Dactylospongia sp., anti-inflammatory evaluation showed that 215-218, and 223 exhibtited potent inhibitory effects on the production of inflammatory cytokines (IL-6, IL-1 $\beta$, IL-8, and PEG2) in LPS-induced THP-1 cells with $\mathrm{IC}_{50}$ values of 5.1-9.2 $\mu \mathrm{M}$ [51]. A new sesquiterpenoid aminoquinone nakijiquinone V (224), along with smenospongine (174) (Figure 3 and Table 2) were extracted from an Indonesian marine Dactylospongia elegans sponge [52]. Eleven new nitrogenous meroterpenoids, cinerols A-K (225-235) (Figure 3 and Table 2), were isolated from the marine sponge Dysideacinerea, 225 and $\mathbf{2 2 6}$ feature a rare 5H-pyrrolo[1,2a]-benzimidazole moiety, while cinerols 227-231 were examples of rare meroterpene benzoxazoles [53]. Six sesquiterpene quinones/hydroquinones (236-240, 210) (Figure 3 and Table 2) were acquired from the marine sponge Dactylospongia elegans [54]. Compounds 238-240 showed activities against the human cancer cell lines DU145, SW1990, Huh7, and PANC-1 with IC 50 values ranging from 2.33 to $37.85 \mu \mathrm{M}$ [54]. Three cytotoxic sesquiterpenoid quinones (241-243) (Figure 3 and Table 2) were purified from South ChinaSea sponge Dysidea sp., and displayed various potent cytotoxic activities with $\mathrm{IC}_{50}$ values ranging from 0.93 to $4.61 \mu \mathrm{M}$ [55]. Two unique nitrogenous sesquiterpene quinone meroterpenoids, dysidinoid B (244) and dysicigyhone A (245) (Figure 3 and Table 2) were characterized from the marine sponge Dysideaseptosa, and 244) exhibited signifcant anti-inflammatory effect by inhibiting TNF- $\alpha$ and IL-6 generation with $\mathrm{IC}_{50}$ values of $9.15 \mu \mathrm{M}$ and17.62 $\mu \mathrm{M}$, respectively [56]. Two nitrogenous merosesquiterpene, 5-epi-nakijiquinone L (246) and 5-epi-smenospongiarine (247) (Figure 3 and Table 2) were isolated from the sponge Verongula cf. rigida with weak $5 \alpha$-reductase inhibitory activity [57].

Drimane sesquiterpenoid-indole alkaloids rarely occur in Nature. Only eight compounds were isolated from actinomycete Streptomyces sp. Three hybrid isoprenoid drimane derivatives-indotertine A (248), drimentine F (249) and drimentine G (250) (Figure 4)—were afforded from a reed rhizosphere soil-derived actinomycete Streptomyces sp. CHQ-64 [58]. Compound 250 showed strong cytotoxicity against human cancer cells lines with $\mathrm{IC}_{50}$ s down to $1.01 \mu \mathrm{M}$, while 248 and 249 showed no significant activity [58]. Four new indolo-drimane sesquiterpenes, dixiamycins A (251) and B (252), oxiamycin (253), and chloroxiamycin (254), were isolated from a marine-derived actinomycete Streptomyces sp. and characterized, together with the known compound xiamycin A (255) (Figure 4) [59]. 251 and 252 are the first examples of atropisomerism of naturally occurring $\mathrm{N}-\mathrm{N}$-coupled atropo-diastereomers, with a dimeric indolo-sesquiterpene skeleton and a stereogenic $\mathrm{N}-\mathrm{N}$ axis between $\mathrm{sp}^{3}$-hybridized nitrogen atoms [59]. The two dimeric compounds 251 and 252 showed better antibacterial activities than the monomers 253-255 with the $\mathrm{IC}_{50}$ values of 4-16 $\mathrm{gg} / \mathrm{mL}$ against four indicator strains (Escherichia coli ATCC25922, Staphylococcus aureus ATCC 29213, Bacillus subtilis SCSIO BS01 and Bacillus thuringiensis SCSIO BT01) [59]. 

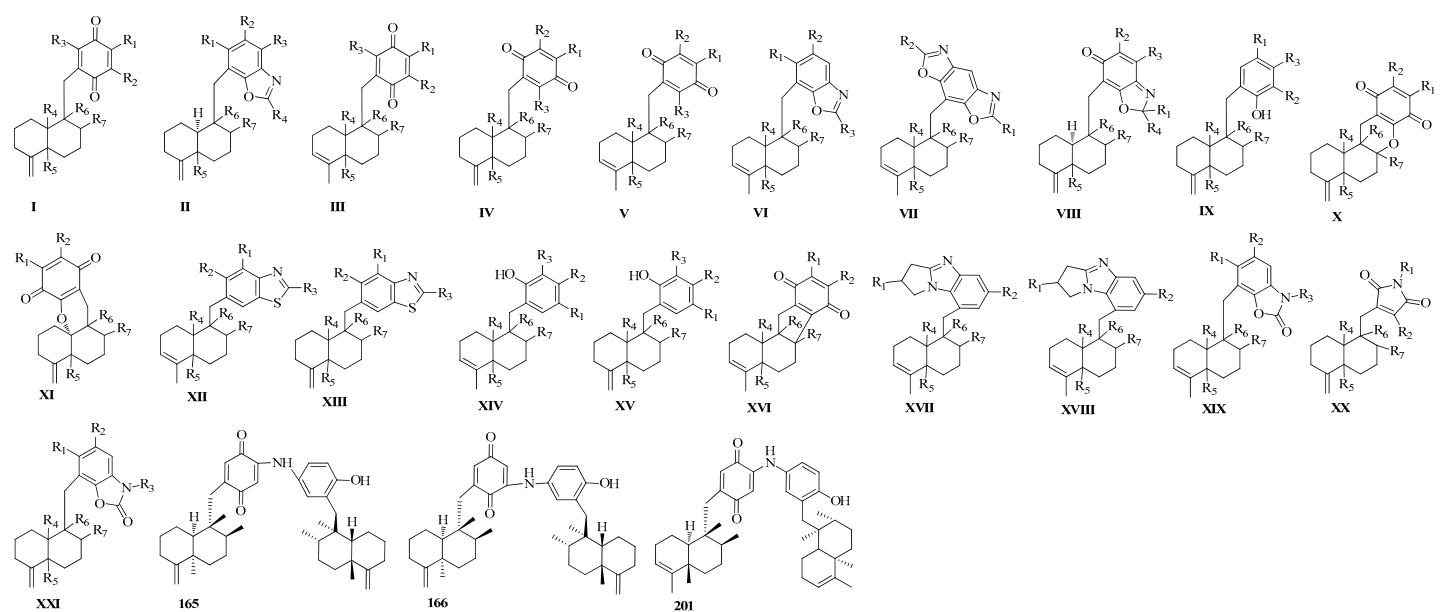

Figure 3. The friedo-drimane sesquiterpenoidskeletons (I-XXI) and three dimers.

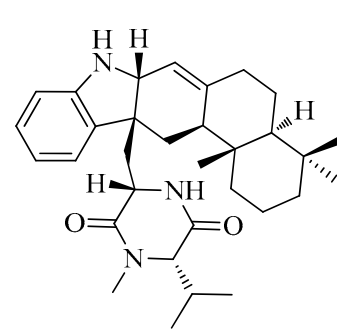

248

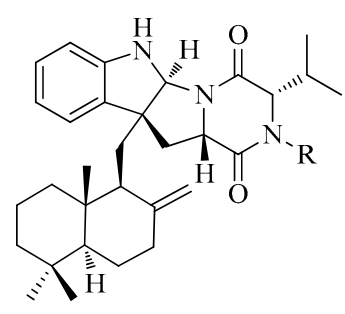

$249 \mathrm{R}=\mathrm{CH} 3$

$250 \mathrm{R}=\mathrm{H}$

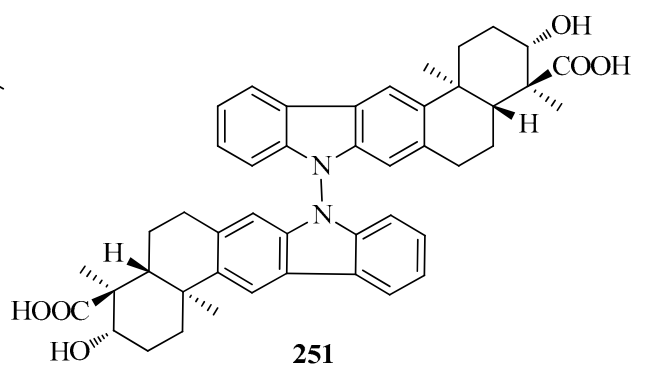<smiles>CCc1cc2c(cc1C)c1ccccc1n2-n1c2ccccc2c2cc3c(cc21)CC[C@@H]1[C@]3(C)CC[C@H](O)[C@]1(C)C(=O)O</smiles>

$\mathrm{COOH}$<smiles>CC1CC[C@@H]2[C@@H](C(=O)O)[C@H](O)CC[C@@]2(C)Oc2cc3c(cc21)[nH]c1ccccc13</smiles>

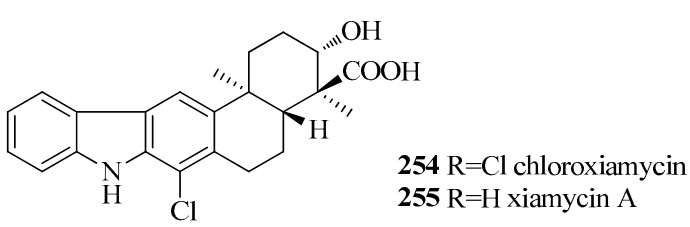

Figure 4. The structures of compounds 248-255. 
Table 2. Reported structures offriedo-drimane sesquiterpenoids.

\begin{tabular}{|c|c|c|c|c|c|c|c|c|c|}
\hline No & Name & $\mathbf{R}_{2}$ & $\mathbf{R}_{3}$ & $\mathbf{R}_{\mathbf{4}}$ & $\mathbf{R}_{5}$ & $\mathbf{R}_{6}$ & $\mathbf{R}_{7}$ & Type & Ref \\
\hline 161 & 18-Aminoarenarone & $\mathrm{NH}_{2}$ & $\mathrm{H}$ & $\alpha \mathrm{H}$ & $\alpha \mathrm{CH}_{3}$ & $\beta \mathrm{CH}_{3}$ & $\mathrm{BCH}_{3}$ & I & [43] \\
\hline 162 & 19-Aminoarenarone & $\mathrm{NH}_{2}$ & $\mathrm{H}$ & $\alpha \mathrm{H}$ & $\alpha \mathrm{CH}_{3}$ & $\beta \mathrm{CH}_{3}$ & $\mathrm{\beta CH}_{3}$ & I & [43] \\
\hline 163 & 18-Methylaminoarenarone & $\mathrm{NHCH}_{3}$ & $\mathrm{H}$ & $\alpha \mathrm{H}$ & $\alpha \mathrm{CH}_{3}$ & $\mathrm{BCH}_{3}$ & $\mathrm{\beta CH}_{3}$ & I & [43] \\
\hline 164 & 19-Methylaminoarenarone & $\mathrm{NHCH}_{3}$ & $\mathrm{H}$ & $\alpha \mathrm{H}$ & $\alpha \mathrm{CH}_{3}$ & $\mathrm{BCH}_{3}$ & $\mathrm{BCH}_{3}$ & I & [43] \\
\hline 167 & Nkijinol B & $\mathrm{OH}$ & $\mathrm{H}$ & $\mathrm{H}$ & $\mathrm{\beta CH}_{3}$ & $\mathrm{\beta CH}_{3}$ & $\mathrm{\beta CH}_{3}$ & II & [44] \\
\hline 168 & Smenospongine B & $\mathrm{NHCH}_{2} \mathrm{COOH}$ & $\mathrm{OH}$ & $\alpha \mathrm{H}$ & $\mathrm{BCH}_{3}$ & $\mathrm{BCH}_{3}$ & $\mathrm{BCH}_{3}$ & $\mathrm{I}$ & [44] \\
\hline 169 & Smenospongine C & $\mathrm{NH}\left(\mathrm{CH}_{2}\right)_{2} \mathrm{COOH}$ & $\mathrm{OH}$ & $\mathrm{H}$ & $\mathrm{\beta CH}_{3}$ & $\mathrm{\beta CH}_{3}$ & $\mathrm{\beta CH}_{3}$ & II & [44] \\
\hline 170 & Nakijinol B diacetate & OAc & $\mathrm{H}$ & $\alpha \mathrm{H}$ & $\mathrm{BCH}_{3}$ & $\mathrm{\beta CH}_{3}$ & $\mathrm{BCH}_{3}$ & $\mathrm{I}$ & [44] \\
\hline 171 & $(-)-3^{\prime}$-Methylaminoavarone & $\mathrm{NHCH}_{3}$ & $\mathrm{H}$ & $\alpha \mathrm{H}$ & $\mathrm{BCH}_{3}$ & $\mathrm{\beta CH}_{3}$ & $\mathrm{BCH}_{3}$ & III & {$[45]$} \\
\hline 172 & (-)-4'-Methylamino-avarone & $\mathrm{NHCH}_{3}$ & $\mathrm{H}$ & $\alpha \mathrm{H}$ & $\mathrm{\beta CH}_{3}$ & $\beta_{\mathrm{CH}}$ & $\mathrm{\beta CH}_{3}$ & III & [45] \\
\hline 173 & (-)-N-Methylmelemeleone-A & $\mathrm{N}\left(\mathrm{CH}_{3}\right)\left(\mathrm{CH}_{2}\right)_{2} \mathrm{SO}_{3} \mathrm{H}$ & $\mathrm{H}$ & $\alpha \mathrm{H}$ & $\mathrm{\beta CH}_{3}$ & $\mathrm{BCH}_{3}$ & $\mathrm{BCH}_{3}$ & III & [45] \\
\hline 174 & Smenospongine & $\mathrm{NH}_{2}$ & $\mathrm{OH}$ & $\alpha \mathrm{H}$ & $\mathrm{BCH}_{3}$ & $\mathrm{BCH}_{3}$ & $\mathrm{BCH}_{3}$ & IV & {$[46,50,52]$} \\
\hline 175 & Glycinylilimaquinone & $\mathrm{NHCH}_{2} \mathrm{COOH}$ & $\mathrm{OH}$ & $\alpha \mathrm{H}$ & $\mathrm{\beta CH}_{3}$ & $\mathrm{\beta CH}_{3}$ & $\mathrm{\beta CH}_{3}$ & IV & [46] \\
\hline 176 & 5-epi-Nakijiquinone S & $\mathrm{H}$ & $\mathrm{OH}$ & $\alpha \mathrm{H}$ & $\alpha \mathrm{CH}_{3}$ & $\mathrm{BCH}_{3}$ & $\mathrm{\beta CH}_{3}$ & $\mathrm{~V}$ & [47] \\
\hline 177 & 5-epi-Nakijiquinone $\mathrm{Q}$ & $\mathrm{H}$ & $\mathrm{OH}$ & $\alpha \mathrm{H}$ & $\alpha \mathrm{CH}_{3}$ & $\mathrm{\beta CH}_{3}$ & $\mathrm{\beta CH}_{3}$ & $\mathrm{~V}$ & [47] \\
\hline 178 & 5-epi-Nakijiquinone $\mathrm{T}$ & $\mathrm{H}$ & $\mathrm{OH}$ & $\alpha \mathrm{H}$ & $\alpha \mathrm{CH}_{3}$ & $\mathrm{\beta CH}_{3}$ & $\mathrm{\beta CH}_{3}$ & $\mathrm{~V}$ & [47] \\
\hline 179 & 5-epi-Nakijiquinone U & $\mathrm{NH}\left(\mathrm{CH}_{2}\right)_{3} \mathrm{SCH}_{3}$ & $\mathrm{OH}$ & $\alpha \mathrm{H}$ & $\alpha \mathrm{CH}_{3}$ & $\mathrm{\beta CH}_{3}$ & $\mathrm{\beta CH}_{3}$ & $\mathrm{~V}$ & [47] \\
\hline 180 & 5-epi-Nakijiquinone $\mathrm{N}$ & $\mathrm{NH}\left(\mathrm{CH}_{2}\right)_{2} \mathrm{CH}\left(\mathrm{CH}_{3}\right)_{2}$ & $\mathrm{OH}$ & $\alpha \mathrm{H}$ & $\alpha \mathrm{CH}_{3}$ & $\mathrm{BCH}_{3}$ & $\mathrm{BCH}_{3}$ & $\mathrm{~V}$ & [47] \\
\hline 181 & 5-epi-Nakijinol C & $\mathrm{OCH}_{3}$ & $\mathrm{CH}_{3}$ & $\alpha \mathrm{H}$ & $\alpha \mathrm{CH}_{3}$ & $\mathrm{BCH}_{3}$ & $\mathrm{BCH}_{3}$ & VI & [47] \\
\hline 182 & 5-epi-Nakijinol D & $\mathrm{CH}_{3}$ & - & $\alpha \mathrm{H}$ & $\alpha \mathrm{CH}_{3}$ & $\beta_{\mathrm{CH}}$ & $\mathrm{\beta CH}_{3}$ & VII & [47] \\
\hline 183 & Dysidaminone A & $\mathrm{NHCH}_{2} \mathrm{CH}\left(\mathrm{CH}_{3}\right)_{2}$ & $\mathrm{H}$ & $\alpha \mathrm{H}$ & $\mathrm{\beta CH}_{3}$ & $\mathrm{\beta CH}_{3}$ & $\mathrm{BCH}_{3}$ & III & [48] \\
\hline 184 & Dysidaminone B & $\mathrm{NHCH}_{2} \mathrm{CH}\left(\mathrm{CH}_{3}\right) \mathrm{CH}_{2} \mathrm{CH}_{3}$ & $\mathrm{H}$ & $\alpha \mathrm{H}$ & $\mathrm{\beta CH}_{3}$ & $\mathrm{\beta CH}_{3}$ & $\mathrm{BCH}_{3}$ & III & [48] \\
\hline 185 & Dysidaminone C & $\mathrm{H}$ & $\mathrm{H}$ & $\alpha \mathrm{H}$ & $\mathrm{BCH}_{3}$ & $\mathrm{BCH}_{3}$ & $\mathrm{BCH}_{3}$ & III & [48] \\
\hline 186 & Dysidaminone D & $\mathrm{N}\left(\mathrm{CH}_{3}\right)_{2}$ & $\mathrm{H}$ & $\alpha \mathrm{H}$ & $\mathrm{BCH}_{3}$ & $\mathrm{BCH}_{3}$ & $\mathrm{BCH}_{3}$ & III & [48] \\
\hline 187 & Dysidaminone $\mathrm{E}$ & $\mathrm{NHCH}_{2} \mathrm{CH}\left(\mathrm{CH}_{3}\right)_{2}$ & $\mathrm{H}$ & $\alpha \mathrm{H}$ & $\mathrm{BCH}_{3}$ & $\mathrm{BCH}_{3}$ & $\mathrm{BCH}_{3}$ & III & [48] \\
\hline 188 & Dysidaminone F & $\mathrm{NHCH}_{2} \mathrm{CH}\left(\mathrm{CH}_{3}\right) \mathrm{CH}_{2} \mathrm{CH}_{3}$ & $\mathrm{H}$ & $\alpha \mathrm{H}$ & $\mathrm{BCH}_{3}$ & $\mathrm{\beta CH}_{3}$ & $\mathrm{BCH}_{3}$ & III & [48] \\
\hline
\end{tabular}


Table 2. Cont.

\begin{tabular}{|c|c|c|c|c|c|c|c|c|c|c|}
\hline No & Name & $\mathbf{R}_{1}$ & $\mathbf{R}_{2}$ & $\mathbf{R}_{3}$ & $\mathbf{R}_{\mathbf{4}}$ & $\mathbf{R}_{5}$ & $\mathbf{R}_{6}$ & $\mathbf{R}_{7}$ & Type & Ref \\
\hline 189 & Dysidaminone G & & $\mathrm{H}$ & $\mathrm{H}$ & $\alpha \mathrm{H}$ & $\mathrm{\beta CH}_{3}$ & $\mathrm{\beta CH}_{3}$ & $\mathrm{\beta CH}_{3}$ & III & [48] \\
\hline 190 & Dysidaminone $\mathrm{H}$ & $\mathrm{H}$ & $\mathrm{NHCH}_{3}$ & $\mathrm{H}$ & $\alpha \mathrm{H}$ & $\mathrm{\beta CH}_{3}$ & $\beta_{C H}$ & $\mathrm{\beta CH}_{3}$ & I & {$[48]$} \\
\hline 191 & Dysidaminone I & $\mathrm{NHCH}_{3}$ & $\mathrm{H}$ & $\mathrm{H}$ & $\alpha \mathrm{H}$ & $\mathrm{\beta CH}_{3}$ & $\mathrm{\beta CH}_{3}$ & $\mathrm{\beta CH}_{3}$ & I & [48] \\
\hline 192 & Dysidaminone J & $\mathrm{H}$ & $\mathrm{N}\left(\mathrm{CH}_{3}\right)_{2}$ & $\mathrm{H}$ & $\alpha \mathrm{H}$ & $\mathrm{BCH}_{3}$ & $\mathrm{BCH}_{3}$ & $\mathrm{\beta CH}_{3}$ & I & [48] \\
\hline 193 & Dysidaminone $\mathrm{K}$ & $\mathrm{NHCH}_{2} \mathrm{CH}\left(\mathrm{CH}_{3}\right)_{2}$ & $\mathrm{H}$ & $\mathrm{H}$ & $\alpha \mathrm{H}$ & $\mathrm{BCH}_{3}$ & $\mathrm{BCH}_{3}$ & $\mathrm{BCH}_{3}$ & I & [48] \\
\hline 194 & Dysidaminone L & $\mathrm{NHCH}_{2} \mathrm{CH}\left(\mathrm{CH}_{3}\right) \mathrm{CH}_{2} \mathrm{CH}_{3}$ & $\mathrm{H}$ & $\mathrm{H}$ & $\alpha \mathrm{H}$ & $\mathrm{BCH}_{3}$ & $\mathrm{BCH}_{3}$ & $\mathrm{BCH}_{3}$ & $\mathrm{I}$ & [48] \\
\hline 195 & Dysidaminone $\mathrm{M}$ & & $\mathrm{H}$ & $\mathrm{H}$ & $\alpha \mathrm{H}$ & $\mathrm{BCH}_{3}$ & $\mathrm{\beta CH}_{3}$ & $\mathrm{\beta CH}_{3}$ & I & [48] \\
\hline 196 & 18-Methylaminoavarone & $\mathrm{H}$ & $\mathrm{NHCH}_{3}$ & $\mathrm{H}$ & $\alpha \mathrm{H}$ & $\beta \mathrm{CH}_{3}$ & $\mathrm{\beta CH}_{3}$ & $\mathrm{\beta CH}_{3}$ & III & [48] \\
\hline 197 & 19-Methylaminoavarone & $\mathrm{NHCH}_{3}$ & $\mathrm{H}$ & $\mathrm{H}$ & $\alpha \mathrm{H}$ & $\mathrm{\beta CH}_{3}$ & $\mathrm{BCH}_{3}$ & $\mathrm{\beta CH}_{3}$ & III & [48] \\
\hline 198 & 18-Aminoavarone & $\mathrm{H}$ & $\mathrm{NH}_{2}$ & $\mathrm{H}$ & $\alpha \mathrm{H}$ & $\mathrm{\beta CH}_{3}$ & $\mathrm{\beta CH}_{3}$ & $\mathrm{\beta CH}_{3}$ & III & [48] \\
\hline 199 & 19-Aminoavarone & $\mathrm{NH}_{2}$ & $\mathrm{H}^{2}$ & $\mathrm{H}$ & $\alpha \mathrm{H}$ & $\mathrm{BCH}_{3}$ & $\mathrm{\beta CH}_{3}$ & $\mathrm{\beta CH}_{3}$ & III & [48] \\
\hline 200 & 18-Phenethylaminoavarone & $\mathrm{H}$ & $\begin{array}{l}\mathrm{N}^{\prime} \\
\mathrm{H}\end{array}$ & $\mathrm{H}$ & $\alpha \mathrm{H}$ & $\mathrm{\beta CH}_{3}$ & $\mathrm{BCH}_{3}$ & $\mathrm{BCH}_{3}$ & III & [48] \\
\hline 201 & Popolohuanone D & & $\mathrm{H}$ & $\mathrm{H}$ & $\alpha \mathrm{H}$ & $\mathrm{\beta CH}_{3}$ & $\mathrm{\beta CH}_{3}$ & $\mathrm{\beta CH}_{3}$ & III & [48] \\
\hline 202 & (-)-Nakijinol E & $\mathrm{OH}$ & $\mathrm{OCH}_{3}$ & $\mathrm{H}$ & $\mathrm{CH}_{3}$ & $\mathrm{BCH}_{3}$ & $\mathrm{\beta CH}_{3}$ & $\mathrm{\beta CH}_{3}$ & II & [49] \\
\hline 203 & (+)-5-epi-Nakijinol E & $\mathrm{OH}$ & $\mathrm{OCH}_{3}$ & $\mathrm{H}$ & $\mathrm{CH}_{3}$ & $\alpha \mathrm{CH}_{3}$ & $\mathrm{\beta CH}_{3}$ & $\mathrm{\beta CH}_{3}$ & II & [49] \\
\hline 204 & Nakijinone A & $\mathrm{CH}_{3}$ & $\mathrm{OCH}_{3}$ & $\mathrm{H}$ & $\mathrm{CH}_{3}$ & $\mathrm{\beta CH}_{3}$ & $\mathrm{\beta CH}_{3}$ & $\mathrm{\beta CH}_{3}$ & VIII & [49] \\
\hline 205 & 5-epi-Nakijinone A & $\mathrm{CH}_{3}$ & $\mathrm{OCH}_{3}$ & $\mathrm{H}$ & $\mathrm{CH}_{3}$ & $\alpha \mathrm{CH}_{3}$ & $\mathrm{\beta CH}_{3}$ & $\mathrm{\beta CH}_{3}$ & VIII & [49] \\
\hline 206 & $\begin{array}{l}\text { 18-Deoxy-18- } \\
\text { formamidodictyoceratin B } \\
\text { 18-Deoxy-18-(2- }\end{array}$ & $\mathrm{COOCH}_{3}$ & $\mathrm{NHCHO}$ & $\mathrm{OH}$ & $\beta \mathrm{H}$ & $\alpha \mathrm{CH}_{3}$ & $\alpha \mathrm{CH}_{3}$ & $\alpha \mathrm{CH}_{3}$ & IX & {$[50]$} \\
\hline 207 & $\begin{array}{c}\text { hydroxyacetyl)aminodictyoceratin } \\
\text { B }\end{array}$ & $\mathrm{COOCH}_{3}$ & $\mathrm{NHCOCH}_{2} \mathrm{OH}$ & $\mathrm{OH}$ & $\beta \mathrm{H}$ & $\alpha \mathrm{CH}_{3}$ & $\alpha \mathrm{CH}_{3}$ & $\alpha \mathrm{CH}_{3}$ & IX & {$[50]$} \\
\hline 208 & N-Methyl-ent-smenospongine & $\mathrm{H}$ & $\mathrm{NHCH}_{3}$ & $\mathrm{OH}$ & $\beta \mathrm{H}$ & $\alpha \mathrm{CH}_{3}$ & $\alpha \mathrm{CH}_{3}$ & $\alpha \mathrm{CH}_{3}$ & I & {$[50]$} \\
\hline 209 & $N$-Methyl-5-epi-smenospongine & $\mathrm{H}$ & $\mathrm{NHCH}_{3}$ & $\mathrm{OH}$ & $\alpha \mathrm{H}$ & $\alpha \mathrm{CH}_{3}$ & $\mathrm{BCH}_{3}$ & $\mathrm{BCH}_{3}$ & I & [50] \\
\hline 210 & $\begin{array}{l}\text { 20-Demethoxy-20- } \\
\text { methylaminodactyloquinone D }\end{array}$ & $\mathrm{H}$ & $\mathrm{NHCH}_{3}$ & - & $\alpha \mathrm{H}$ & $\mathrm{BCH}_{3}$ & $\mathrm{\beta CH}_{3}$ & $\mathrm{\beta CH}_{3}$ & $x$ & {$[50,54]$} \\
\hline
\end{tabular}


Table 2. Cont

\begin{tabular}{|c|c|c|c|c|c|c|c|c|c|c|}
\hline No & Name & $\mathbf{R}_{\mathbf{1}}$ & $\mathbf{R}_{\mathbf{2}}$ & $\mathbf{R}_{3}$ & $\mathbf{R}_{\mathbf{4}}$ & $\mathbf{R}_{\mathbf{5}}$ & $\mathbf{R}_{6}$ & $\mathbf{R}_{7}$ & Type & Ref \\
\hline 211 & $\begin{array}{l}\text { 20-Demethoxy-20-methylamino- } \\
\text { 5-epidactylo-quinone D }\end{array}$ & $\mathrm{H}$ & $\mathrm{NHCH}_{3}$ & - & $\alpha \mathrm{H}$ & $\mathrm{BCH}_{3}$ & $\mathrm{BCH}_{3}$ & $\mathrm{\beta CH}_{3}$ & IV & {$[50]$} \\
\hline 212 & $\begin{array}{l}\text { 20-Demethoxy-20- } \\
\text { methylaminodactyloquinone B }\end{array}$ & $\mathrm{H}$ & $\mathrm{NHCH}_{3}$ & - & - & $\alpha \mathrm{CH}_{3}$ & $\mathrm{BCH}_{3}$ & $\mathrm{BCH}_{3}$ & XI & {$[50]$} \\
\hline 213 & 5-epi-Smenospongine & $\mathrm{H}$ & $\mathrm{NH}_{2}$ & $\mathrm{OH}$ & $\alpha \mathrm{H}$ & $\alpha \mathrm{CH}_{3}$ & $\mathrm{\beta CH}_{3}$ & $\mathrm{\beta CH}_{3}$ & IV & {$[50]$} \\
\hline 214 & Smenospongiadine & $\mathrm{H}$ & & $\mathrm{OH}$ & $\alpha \mathrm{H}$ & $\mathrm{BCH}_{3}$ & $\mathrm{\beta CH}_{3}$ & $\mathrm{BCH}_{3}$ & IV & {$[50]$} \\
\hline 215 & Dactylospongin A & $\mathrm{H}$ & $\mathrm{OH}$ & $\mathrm{H}$ & $\beta \mathrm{H}$ & $\alpha \mathrm{CH}_{3}$ & $\alpha \mathrm{CH}_{3}$ & $\alpha \mathrm{CH}_{3}$ & XII & [51] \\
\hline 216 & Dactylospongin B & $\mathrm{H}$ & $\mathrm{OH}$ & $\mathrm{H}$ & $\alpha \mathrm{H}$ & $\mathrm{\beta CH}_{3}$ & $\mathrm{\beta CH}_{3}$ & $\mathrm{\beta CH}_{3}$ & XIII & [51] \\
\hline 217 & Dactylospongin C & NHCHO & $\mathrm{H}$ & $\mathrm{H}$ & $\beta \mathrm{H}$ & $\alpha \mathrm{CH}_{3}$ & $\alpha \mathrm{CH}_{3}$ & $\alpha \mathrm{CH}_{3}$ & XIV & [51] \\
\hline 218 & Dactylospongin D & $\mathrm{NHCHO}$ & $\mathrm{H}$ & $\mathrm{H}$ & $\alpha \mathrm{H}$ & $\mathrm{\beta CH}_{3}$ & $\mathrm{\beta CH}_{3}$ & $\mathrm{BCH}_{3}$ & $\mathrm{XV}$ & [51] \\
\hline 219 & ent-Melemeleone B & $\mathrm{NHCH}_{2} \mathrm{CH}_{2} \mathrm{SO}_{3} \mathrm{H}$ & $\mathrm{H}$ & $\mathrm{H}$ & $\beta \mathrm{H}$ & $\alpha \mathrm{CH}_{3}$ & $\alpha \mathrm{CH}_{3}$ & $\alpha \mathrm{CH}_{3}$ & $\mathrm{~V}$ & [51] \\
\hline 220 & Melemeleone C & $\mathrm{H}$ & $\mathrm{NHCH}_{2} \mathrm{CH}_{2} \mathrm{SO}_{3} \mathrm{H}$ & $\mathrm{H}$ & $\beta \mathrm{H}$ & $\alpha \mathrm{CH}_{3}$ & $\alpha \mathrm{CH}_{3}$ & $\alpha \mathrm{CH}_{3}$ & $\mathrm{~V}$ & [51] \\
\hline 221 & Melemeleone D & $\mathrm{NHCH}_{2} \mathrm{CH}_{2} \mathrm{SO}_{3} \mathrm{H}$ & $\mathrm{H}$ & $\mathrm{H}$ & $\alpha \mathrm{H}$ & $\mathrm{\beta CH}_{3}$ & $\mathrm{\beta CH}_{3}$ & $\mathrm{BCH}_{3}$ & IV & [51] \\
\hline 222 & Melemeleone E & $\mathrm{H}$ & $\mathrm{NHCH}_{2} \mathrm{CH}_{2} \mathrm{SO}_{3} \mathrm{H}$ & - & $\alpha \mathrm{H}$ & $\mathrm{BCH}_{3}$ & $\mathrm{BCH}_{3}$ & $\mathrm{\beta CH}_{3}$ & XVI & [51] \\
\hline 223 & Dysidaminone $\mathrm{N}$ & $\mathrm{H}$ & $\mathrm{H}$ & $\mathrm{H}$ & $\alpha \mathrm{H}$ & $\mathrm{BCH}_{3}$ & $\mathrm{\beta CH}_{3}$ & $\mathrm{\beta CH}_{3}$ & IV & [51] \\
\hline 224 & Nakijiquinone V & $\mathrm{H}$ & $\begin{array}{l}\mathrm{N} \\
\mathrm{H}\end{array}$ & $\mathrm{OH}$ & $\alpha \mathrm{H}$ & $\mathrm{\beta CH}_{3}$ & $\mathrm{BCH}_{3}$ & $\beta \mathrm{CH}_{3}$ & IV & [52] \\
\hline 225 & Cinerol A & $\mathrm{H}$ & $\mathrm{OH}$ & - & $\alpha \mathrm{H}$ & $\mathrm{\beta CH}_{3}$ & $\mathrm{\beta CH}_{3}$ & $\mathrm{\beta CH}_{3}$ & XVII & [53] \\
\hline 226 & Cinerol B & $\mathrm{H}$ & $\mathrm{OH}$ & - & $\alpha \mathrm{H}$ & $\mathrm{\beta CH}_{3}$ & $\beta_{\mathrm{CH}}$ & $\mathrm{\beta CH}_{3}$ & XVIII & [53] \\
\hline 227 & Cinerol C & $\mathrm{H}$ & $\mathrm{OH}$ & $\mathrm{H}$ & $\alpha \mathrm{H}$ & $\mathrm{\beta CH}_{3}$ & $\mathrm{\beta CH}_{3}$ & $\mathrm{\beta CH}_{3}$ & VI & [53] \\
\hline 228 & Cinerol D & $\mathrm{H}$ & $\mathrm{OH}$ & $\mathrm{CH}_{3}$ & $\alpha \mathrm{H}$ & $\mathrm{\beta CH}_{3}$ & $\mathrm{\beta CH}_{3}$ & $\mathrm{BCH}_{3}$ & VI & [53] \\
\hline 229 & Cinerol E & $\mathrm{H}$ & $\mathrm{OH}$ & $\mathrm{H}$ & $\mathrm{CH}_{3}$ & $\mathrm{\beta CH}_{3}$ & $\mathrm{\beta CH}_{3}$ & $\mathrm{BCH}_{3}$ & II & [53] \\
\hline 230 & Cinerol F & $\mathrm{H}$ & $\mathrm{OH}$ & $\mathrm{H}$ & $\alpha \mathrm{H}$ & $\mathrm{\beta CH}_{3}$ & $\mathrm{\beta CH}_{3}$ & $\mathrm{\beta CH}_{3}$ & XIX & [53] \\
\hline 231 & Cinerol G & $\mathrm{H}$ & $\mathrm{OH}$ & $\mathrm{CH}_{3}$ & $\alpha \mathrm{H}$ & $\mathrm{BCH}_{3}$ & $\mathrm{BCH}_{3}$ & $\mathrm{\beta CH}_{3}$ & XIX & [53] \\
\hline 232 & Cinerol H & & $\mathrm{H}$ & $\mathrm{H}$ & $\alpha \mathrm{H}$ & $\mathrm{\beta CH}_{3}$ & $\mathrm{\beta CH}_{3}$ & $\mathrm{\beta CH}_{3}$ & XIV & [53] \\
\hline 233 & Cinerol I & & $\mathrm{H}$ & $\mathrm{H}$ & $\alpha \mathrm{H}$ & $\mathrm{\beta CH}_{3}$ & $\mathrm{\beta CH}_{3}$ & $\mathrm{\beta CH}_{3}$ & XIV & [53] \\
\hline
\end{tabular}


Table 2. Cont.

\begin{tabular}{|c|c|c|c|c|c|c|c|c|c|c|}
\hline No & Name & $\mathbf{R}_{\mathbf{1}}$ & $\mathbf{R}_{\mathbf{2}}$ & $\mathbf{R}_{3}$ & $\mathbf{R}_{4}$ & $\mathbf{R}_{5}$ & $\mathbf{R}_{6}$ & $\mathbf{R}_{7}$ & Type & Ref \\
\hline 234 & Cinerol J & $\mathrm{NHCHO}$ & $\mathrm{H}$ & $\mathrm{H}$ & $\alpha \mathrm{H}$ & $\mathrm{\beta CH}_{3}$ & $\mathrm{\beta CH}_{3}$ & $\mathrm{\beta CH}_{3}$ & XIV & [53] \\
\hline 235 & Cinerol K & $\mathrm{NHCOCH}_{2} \mathrm{CH}\left(\mathrm{CH}_{3}\right)_{2}$ & $\mathrm{H}$ & $\mathrm{H}$ & $\alpha \mathrm{H}$ & $\mathrm{\beta CH}_{3}$ & $\mathrm{\beta CH}_{3}$ & $\mathrm{\beta CH}_{3}$ & XIV & [53] \\
\hline 236 & $\begin{array}{l}\text { 20-Demethoxy-20- } \\
\text { isopentylaminodactyloquinone D }\end{array}$ & $\mathrm{H}$ & $\mathrm{NH}\left(\mathrm{CH}_{2}\right)_{2} \mathrm{CH}\left(\mathrm{CH}_{3}\right)_{2}$ & - & $\alpha \mathrm{H}$ & $\mathrm{BCH}_{3}$ & $\mathrm{\beta CH}_{3}$ & $\mathrm{BCH}_{3}$ & $x$ & [54] \\
\hline 237 & $\begin{array}{l}\text { 20-Demethoxy-20- } \\
\text { isobutylaminodactyloquinone D }\end{array}$ & $\mathrm{H}$ & $\mathrm{NHCH}_{2} \mathrm{CH}\left(\mathrm{CH}_{3}\right)_{2}$ & - & $\alpha \mathrm{H}$ & $\mathrm{\beta CH}_{3}$ & $\mathrm{\beta CH}_{3}$ & $\mathrm{\beta CH}_{3}$ & $x$ & [54] \\
\hline 238 & Smenospongiarine & $\mathrm{H}$ & $\mathrm{NH}\left(\mathrm{CH}_{2}\right)_{2} \mathrm{CH}\left(\mathrm{CH}_{3}\right)_{2}$ & $\mathrm{OH}$ & $\beta \mathrm{H}$ & $\alpha \mathrm{CH}_{3}$ & $\alpha \mathrm{CH}_{3}$ & $\alpha \mathrm{CH}_{3}$ & I & [54] \\
\hline 239 & Smenospongorine & $\mathrm{H}$ & $\mathrm{NHCH}_{2} \mathrm{CH}\left(\mathrm{CH}_{3}\right)_{2}$ & $\mathrm{OH}$ & $\beta \mathrm{H}$ & $\alpha \mathrm{CH}_{3}$ & $\alpha \mathrm{CH}_{3}$ & $\alpha \mathrm{CH}_{3}$ & I & [54] \\
\hline 240 & Smenospongimine & $\mathrm{H}$ & $\mathrm{NHCH}_{3}$ & $\mathrm{OH}$ & $\beta \mathrm{H}$ & $\alpha \mathrm{CH}_{3}$ & $\alpha \mathrm{CH}_{3}$ & $\alpha \mathrm{CH}_{3}$ & I & [54] \\
\hline 241 & (+)-19-Methylaminoavarone & $\mathrm{NHCH}_{3}$ & $\mathrm{H}$ & $\mathrm{H}$ & $\alpha \mathrm{H}$ & $\mathrm{BCH}_{3}$ & $\mathrm{BCH}_{3}$ & $\mathrm{\beta CH}_{3}$ & V & [55] \\
\hline 242 & (-)-20-Phenethylaminoavarone & $\mathrm{H}$ & ${ }_{\mathrm{N}}^{\mathrm{H}} / \overbrace{}^{2}$ & $\mathrm{H}$ & $\alpha \mathrm{H}$ & $\mathrm{BCH}_{3}$ & $\mathrm{\beta CH}_{3}$ & $\mathrm{\beta CH}_{3}$ & $\mathrm{~V}$ & [55] \\
\hline 243 & (-)-20-Methylaminoavarone & $\mathrm{H}$ & $\mathrm{NHCH}_{3}$ & $\mathrm{H}$ & $\alpha \mathrm{H}$ & $\mathrm{\beta CH}_{3}$ & $\mathrm{\beta CH}_{3}$ & $\mathrm{\beta CH}_{3}$ & $\mathrm{~V}$ & [55] \\
\hline 244 & Dysidinoid B & $\mathrm{H}$ & $\mathrm{H}$ & - & $\alpha \mathrm{H}$ & $\mathrm{BCH}_{3}$ & $\mathrm{BCH}_{3}$ & $\mathrm{BCH}_{3}$ & $x x$ & {$[56]$} \\
\hline 245 & Dysicigyhone A & $\mathrm{H}$ & $\mathrm{OH}$ & $\mathrm{CH}_{3}$ & $\alpha \mathrm{H}$ & $\mathrm{\beta CH}_{3}$ & $\mathrm{\beta CH}_{3}$ & $\mathrm{\beta CH}_{3}$ & XXI & [56] \\
\hline 246 & 5-epi-Nakijiquinone L & $\mathrm{H}$ & $\mathrm{NHCH}_{2} \mathrm{CH}\left(\mathrm{CH}_{3}\right) \mathrm{CH}_{2} \mathrm{CH}_{3}$ & $\mathrm{OH}$ & $\alpha \mathrm{H}$ & $\alpha \mathrm{CH}_{3}$ & $\mathrm{BCH}_{3}$ & $\mathrm{BCH}_{3}$ & IV & [57] \\
\hline 247 & 5-epi-Smenospongiarine & $\mathrm{H}$ & $\mathrm{NH}\left(\mathrm{CH}_{2}\right)_{2} \mathrm{CH}\left(\mathrm{CH}_{3}\right)_{2}$ & $\mathrm{OH}$ & $\alpha \mathrm{H}$ & $\alpha \mathrm{CH}_{3}$ & $\mathrm{BCH}_{3}$ & $\mathrm{BCH}_{3}$ & IV & [57] \\
\hline
\end{tabular}




\subsection{Eudesmane Sesquiterpenoids}

Eleven nitrogen-containing eudesmane sesquiterpenoids, halichonadins G-Q (256-266) (Figure 5), were isolated from a marine sponge Halichondria sp., and compounds 256 and 258 showed cytotoxicity against murine lymphoma L1210 cells ( $\mathrm{IC}_{50} 5.9$ and $6.9 \mu \mathrm{g} / \mathrm{mL}$ )and human epidermoid carcinoma $\mathrm{KB}$ cells ( $\mathrm{IC}_{50} 6.7$ and $3.4 \mu \mathrm{g} / \mathrm{mL}$ ) in vitro, Halichonadin $\mathrm{K}$ showed cytotoxicity against human epidermoid carcinoma $\mathrm{KB}$ cells $\left(\mathrm{IC}_{50} 10.6 \mu \mathrm{g} / \mathrm{mL}\right)$ in vitro, and halichonadin $\mathrm{O}$ displayed antimicrobial activity against Staphylococcus aureus (MIC $8 \mu \mathrm{g} / \mathrm{mL}$ ), Micrococcus luteus (MIC $8 \mu \mathrm{g} / \mathrm{mL}$ ), and Trichophyton mentagrophytes ( $\mathrm{IC}_{50} 16 \mu \mathrm{g} / \mathrm{mL}$ ) [60-62]. One eudesmane-type sesquiterpene, phaeusmane I (267) (Figure 5), was isolatedfrom the rhizomes of Curcuma phaeocaulis [63]. Three new nitrogen-containing sesquiterpenoids, the cespilamides C-E (268-270, Figure 5) were purified from the Taiwanese soft coral Cespitularia taeniata, and 270 exhibited cytotoxicity against human breast adenocarcinoma (MCF-7), medulloblastoma (Daoy), and cervical epitheloid carcinoma (Hela) cancer cells with $\mathrm{IC}_{50}$ of $17.5,22.3$, and $24.7 \mu \mathrm{M}$, respectively [64]. Acanthine B (271), acanthine C (272), 11-isocyano-7 $\beta H$-eudesm-5-ene (273), 11-isothiocyano-7 $\beta H$-eudesm-5-ene (274), and 11-formamido-7 $\beta H$-eudesm-5-ene (275) (Figure 5), were isolated from the Thai sponge Halichondria sp. [65]. Four new uncommon nitrogenous eudesmane-type sesquiterpenes, axiriabilines A-D (276-279), and one known related ent-stylotelline (280) (Figure 5), were isolated from the Hainan sponge Axinyssa variabilis with no cytotoxicity against several cancer cells [66]. Axiriabiline A (276) and 11-formamido-7 $\beta$ H-eudesm-5-ene (281) (Figure 5) were extracted from South China Sea Nudibranchs Phyllidiella sp. [67]. Spiroalanpyrroids A (282) and B (283), two sesquiterpene alkaloids with an unprecedented eudesmanolide-pyrrolizidine spiro [55] framework, were isolated together with two new sesquiterpene-amino acidadducts, helenalanprolines A (284) and B (285) (Figure 5), from the roots of Inula helenium [68]. Bioassays showed that $\mathbf{2 8 4}$ and 285 significantly inhibited nitric oxide production in lipopolysaccharide-induced RAW 264.7 macrophages with $\mathrm{IC}_{50}$ values of 15.8 and $13.5 \mu \mathrm{M}$, respectively [68].

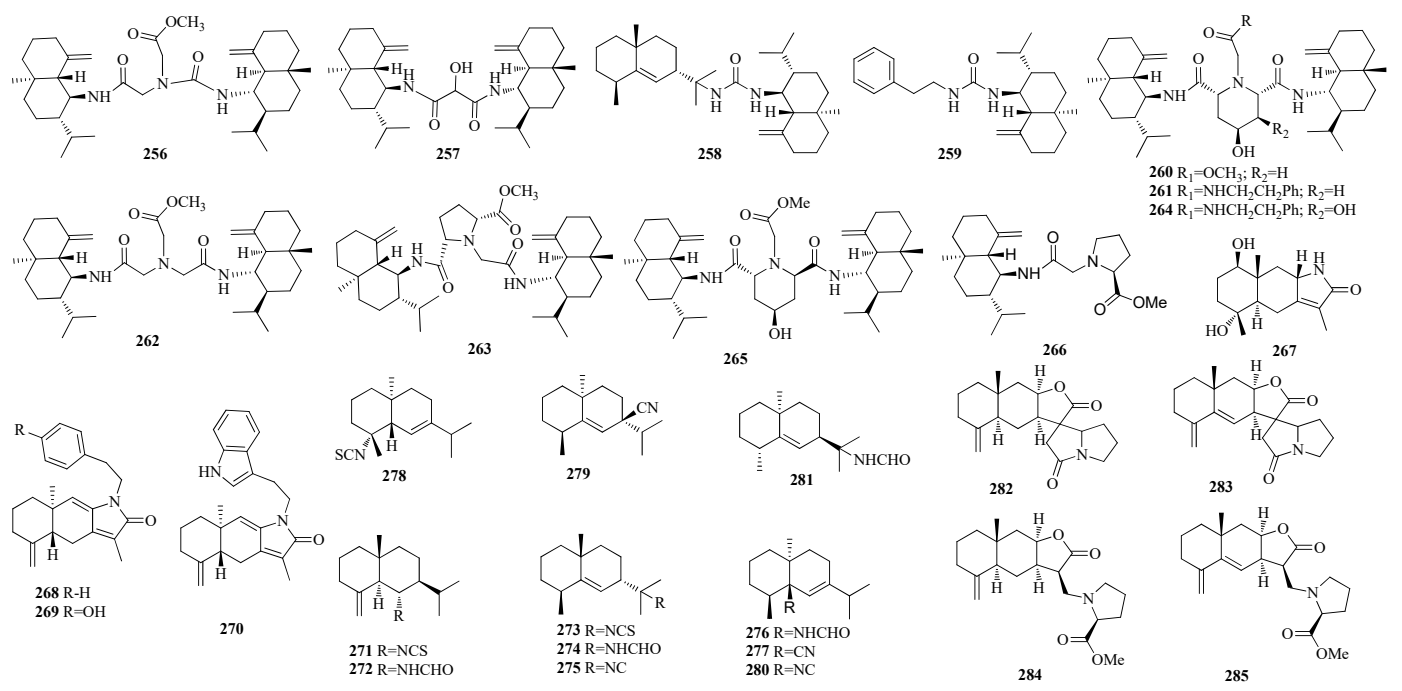

Figure 5. The structures of compounds 256-285.

\subsection{Cadinane Sesquiterpenoids}

Two nitrogenous cadinane sesquiterpenes $\left(3 S^{*}, 5 R^{*}, 6 R^{*}, 9 R^{*}\right)$-3-formamido-1(10)-cadinene (286) and (-)-halichamine (287) (Figure 6) were isolated from the Thai marine sponge Halichondria sp. [69]. Compound 286 showed moderate cytotoxic activity against HeLa, MOLT-3, and HepG2 cell lines with $\mathrm{IC}_{50}$ valued of 32.1, 33.4, and $16.0 \mathrm{mM}$, respectively, while compound 287 also displayed moderate cytotoxic activity against HuCCA-1, MOLT-3, HepG2, and MDA-MB231 cell lines with $\mathrm{IC}_{50}$ valued of 20.3, 34.6, 19.9, and 22.6 mM, respectively [69]. (1R, 6S, 7S, 10S)-10-isothiocyanato-4-amorphene (288), 
axinisothiocyanate J (289) (Figure 6) were extracted from the marine sponge Axinyssa sp. [70]. Halichon C (290) and 4-epihalichon C (291), halichon D (292), halichonG (293), (-)-10-isocyano-4-cadinene (294), and (-)-10-isothiocyanato-4-cadinene (295) (Figure 6), were obtained from the Thai sponge Halichondria sp. [65]. Compounds 290, 291, and 294 exhibited moderate cytotoxicity ( $\mathrm{IC}_{50} 20.9$, 29.0, and $9.1 \mu \mathrm{M}$, respectively) against the MOLT-3 cell line and compound 292 also showed moderate cytotoxicity against HepG2 and MDA-MB-231 cell lines with $\mathrm{IC}_{50}$ values of 24.3 and $19.3 \mu \mathrm{M}$, respectively [65]. New stereoisomers of $(+)-\left(1 S^{*}, 4 S^{*}, 6 S^{*}, 7 R^{*}\right)-4-$ Isocyano-9-amorphene (296) and of $(-)-\left(1 S^{*}, 6 R^{*}, 7 R^{*}, 10 S^{*}\right)$-10-isocyano-4-amorphene (297), $4 \alpha$-isocyano-9-amorphene (298), $\left(1 S^{*}, 4 S^{*}, 6 S^{*}, 7 R^{*}\right)$-4-thiocyanate-9-cadinene (299), (-)-10-isocyano-4-amorphene (300), (-)-10-isothiocyanato-4-cadinene (301) (Figure 6), were identified from Phyllidiella pustulosa and from Phyllidia ocellata [71]. A novel sesquiterpenoidal lactam, commipholactam A (302) (Figure 6) was isolated from Resina commiphora [72]. Biological assessment against human cancer cells showed that the $\mathrm{IC}_{50}$ values of 302 against HepG2 and A549 cells were $21.73 \mu \mathrm{M}$ and $128.50 \mu \mathrm{M}$, respectively [72]. Axidaoisocyanate A (303), 10-isothiocyanato-4-cadinene (304), 10-formamido-4-cadinene (305), along with 289, 293 (Figure 6), were identified from two South China Sea Nudibranchs Phyllidiella pustulosa, Phyllidia coelestis [67].<smiles>[R][C@]1(C)CC=C2[C@@H](C)CC[C@H](C(C)C)[C@H]2C1</smiles>

$286 \mathrm{R}=\mathrm{NHCHO}$ $287 \mathrm{R}=\mathrm{NH}_{2}$<smiles>CC1=C[C@H]2CCC(C(C)C)[C@H](C)[C@H]2CC[C@H]1C</smiles>

$294 \mathrm{R}=\mathrm{NC}$ $295 \mathrm{R}=\mathrm{NCS}$<smiles>Cc1ccc2c(C)cc3c(c2c1)[C@H](O)C(=O)N3</smiles>

302<smiles>CC1=C[C@H]2C(C(C)C)CC[C@H](C#N)[C@H]2CC1</smiles>

288<smiles>[Z]C1CC[C@H]2C(C)=CC[C@H](C(C)C)[C@H]2C1</smiles>

$296 \mathrm{R}=\mathrm{NC}$ $299 \mathrm{R}=\mathrm{NCS}$<smiles>CC(C)[C@H]1CC[C@H](C)[C@]2(C)CC[C@](C)(O)C=C12</smiles>
303<smiles>CC(C)C1CC[C@@H](C#N)[C@H]2CC[C@](C)(O)C=C12</smiles>

289<smiles>[R]C1CC[C@H](C(C)C)C2=C[C@](C)(O)CC[C@H]2[C@H]1C</smiles>

$290 \mathrm{R}=\mathrm{NC}$ $293 \mathrm{R}=\mathrm{NHCHO}$<smiles>CC1=C[C@H]2C(C(C)C)CC[C@H](C)[C@H]2CC1</smiles>

297<smiles>CC1=CC[C@H](C(C)C)[C@H]2C[C@@](C)(C#N)CC[C@H]12</smiles>

298<smiles>[Y4][C@H]1CC[C@H](C(C)C)C2=C[C@](C)(O)CC[C@H]21</smiles>

291<smiles>CC1=C[C@H]2C(C(C)C)CC[C@](C)(N)[C@]2(C)CC1</smiles>

300<smiles>CC1=CC[C@H](C(C)C)[C@H]2CC[C@](C)(C#N)C[C@H]12</smiles>

292<smiles>CC1=C[C@H]2C(C(C)C)CC[C@H](Cl)[C@H]2CC1</smiles>

301

Figure 6. The structures of compounds 286-305.

\subsection{Bisabolane Sesquiterpenoids}

Brasilamides E-J (306-311), bisabolane sesquiterpenoids with 3-cyclohexylfuran (306 and 307) and 3-cyclohexylfuranone (308-311) skeletons (Figure 7), were isolated from scaled-up fermentation cultures of the plant endophytic fungus Paraconiothynium brasiliense Verkley [73]. Compound 307 selectively inhibited the proliferation of the breast (MCF-7) and gastric (MGC) cancer cell lines, with $\mathrm{IC}_{50}$ values of 8.4 and $14.7 \mu \mathrm{M}$, respectively [73]. N,N'-bis[(6R,7S)-7-amino-7,8-dihydro-a-bisabolen-7-yl]urea (304), and (6R,7S)-7-amino-7,8-dihydro- $\alpha$-bisabolene (313) (Figure 7), were purified from the marine sponge Axinyssa sp. collected atIriomote Island [70]. Compound 312 was the most potent inhibitor of PTP1B activity $\left(\mathrm{IC}_{50}=1.9 \mu \mathrm{M}\right)$ without cytotoxicity at $50 \mu \mathrm{M}$ in two human cancer cell lines, hepatoma Huh-7 and bladder carcinoma EJ-1 cells [70]. Compound 312 also moderately enhanced the insulin-stimulated phosphorylation levels of Aktin Huh-7 cells [70]. D ${ }^{7,14}$-3-isocyanotheonellin (314) and 3-isocyanotheonellin (315), theonellin formamide (316), theonellin isothiocyanate (317), and 7-isocyano-7,8-dihydro- $\alpha$-bisabolene (318) (Figure 7) were extracted from the two South China Sea 
nudibranchs Phyllidiella pustulosa and Phyllidia coelestis [67]. Compounds 315, 317, and 318 exhibited strong cytotoxicity against human cancercell line SNU-398 with $\mathrm{IC}_{50}$ values of $0.50,2.15$, and $0.50 \mu \mathrm{M}$, respectively [67]. In addition, compound 315 also displayed broad cytotoxicity against the other three cancer cell lines, including A549, HT-29, and Capan-1, with $\mathrm{IC}_{50}$ values of 8.60, 3.35, and $1.98 \mu \mathrm{M}$, respectively [67]. A rearranged bisabolene-type sesquiterpene, halichonic acid (319), was isolated from a marine sponge Halichondria sp., together with 313 [74] (Figure 7). Compound 313 was cytotoxic against HeLa cells with an $\mathrm{IC}_{50}$ value of $50 \mu \mathrm{M}$, whereas 314 did not show cytotoxicity even at $50 \mu \mathrm{M}$ [74]. Five novel highly oxygenated norbisabolane sesquiterpene, namely phyllanthacidoid U (320), phyllanthacidoidA (321), phyllanthacidoid B (322), phyllanthacidoid L (323), and phyllanthacidoid S (324) (Figure 7) were isolated from the roots and stems of Phyllanthus acidus, and compounds 321-323 displayed potential anti hepatitis B virus (anti-HBV) activities [75].

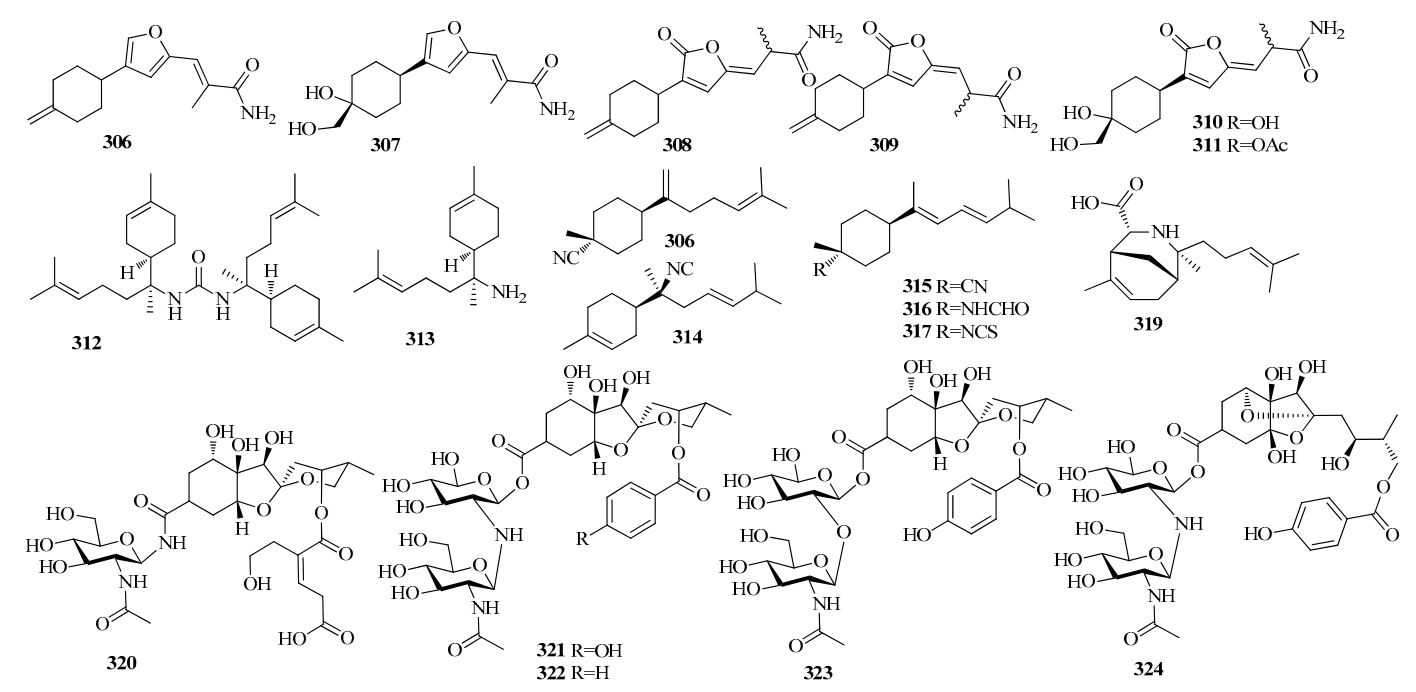

Figure 7. The structures of compounds 306-324.

\subsection{Germacrane, Elemaneand Iresane Sesquiterpenoids}

Two germacrane-type sesquiterpenoid dimers-isobisparthenolidine (325) and bisparthenolidine (326) (Figure 8) were isolated from the chloroform-soluble fraction of the methanolic extract of the bark of Magnolia kobus (Magnoliaceae) [76]. Compound 325 displayed broad cytotoxicity against four cancer cell lines, including A549, SK-OV-3, SK-MEL-2, and HCT-15, with $\mathrm{IC}_{50}$ values of 2.0, 1.9, 3.9 and $3.2 \mu \mathrm{M}$, respectively [76]. Noveliresane sesquiterpene alkaloids, halichonines A (327), B (328), and C (329) (Figure 8), were identified from the marine sponge Halichondria okadai Kadota, and 328 wasthen subjected to the trypan blue dye exclusion using HL60 human leukemia cells, and showed cytotoxicity ( $\mathrm{IC}_{50}$ value: $0.60 \mu \mathrm{g} / \mathrm{mL}$ ) [77]. One $\gamma$-elemene-type sesquiterpenes, $8 \beta(H)$-elema-1,3,7(11)-trien-8,12-lactam (330) (Figure 8) was obtained from the rhizomes of Curcuma phaeocaulis [63]. Three new germacrane sesquiterpenoid-typealkaloids with an unusual $\Delta^{8}$-7,12-lactam moiety, glechomanamides A-C (331-333) (Figure 8) were isolated from Salvia scapiformis [78]. In a tube formation assay, 332 showed the most potent antiangiogenic activity in primary screening, and its $\mathrm{IC}_{50}$ value was determined to be $40.4 \mu \mathrm{M}$ [78]. In addition to VEGFR2, 332 decreased BMP4 expression, which regulates tube formation, and glycolysisrelated proteins, including GLUT1 and HK2, which suggests that the novel compound 332 is worthy of additional investigation for angiogenesis-associated pathological conditions [78]. Onopornoids A-D (334-337) (Figure 8), three elemanes and one germacrane, were extracted from the whole aerial parts of Onopordum alexandrinum, which possess unique structures combining a sesquiterpenoid framework with an amino acid, L-proline [79]. 


\subsection{Farnesane, Spiroaxane, Aromadendrane and Pupukeanane Sesquiterpenoids}

Chemical investigation of the endophytic fungus Emericella sp. (HK-ZJ) isolated from the mangrove plant Aegiceras corniculatum led to the isolation of six farnesane sesquiterpenoids named emeriphenolicins A-F (338-343) (Figure 9) with moderate anti-influenza A viral (H1N1) activities [80]. An unusual farnesane natural product (dotofide, 344) (Figure 9), in which the terpenoid skeleton is interrupted by a guanidine moiety was obtained from the marine slug Doto pinnatifida [81]. Two spiroaxane sesquiterpenes, (-)-axisonitrile-3 (345), (+)-axamide-3 (346), and one aromadendrane sesquiterpene axamide-2 (347) (Figure 9) were isolated from the Thai marine sponge Halichondria sp., and only 345 showed strong activity to the HepG2 cell line withan IC $_{50}$ value of $1.3 \mu \mathrm{M}$ [69]. Fasciospyrinadine (348) (Figure 9), a novel farnesane sesquiterpene pyridine alkaloid was extracted froma Guangxi sponge Fasciospongia sp. [82].<smiles></smiles>

325<smiles>C=CC1(C)C[C@H](OC(=O)CCO)[C@@H]2[C@@H](OC(=O)[C@H]2CN2CCC[C@H]2C(=O)O)C1C(=C)CO</smiles>

334<smiles>C=CC1(C)C[C@H](OC(=O)/C(=C\O)CON2CCC[C@H]2C(=O)O)[C@H](O)[C@H]2C(=O)O[C@@H]21</smiles>

336

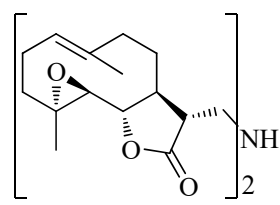

326<smiles>C=CC1(C)C[C@H](OC(=O)C(=C)CO)[C@@H]2[C@@H](OC(=O)[C@H]2CN2CCC[C@H]2C(=O)O)[C@H]1C(=C)CO</smiles>

335<smiles>C=C(CO)C(=O)O[C@H]1CC2=CCCC(CO)=C2C[C@H]1CN1CCC[C@H]1C(=O)O</smiles>

337

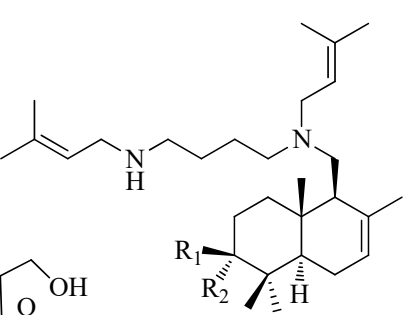

$327 \mathrm{R}_{1}=\mathrm{OH} ; \mathrm{R}_{2}=\mathrm{H}$

$\begin{array}{ll}328 \mathrm{R}_{1}=\mathrm{H} ; \mathrm{R}_{2}=\mathrm{H} & 332 \mathrm{R}=\mathrm{R}=\mathrm{Tig} ; \mathrm{R}_{2}=\mathrm{H} \\ 329 \mathrm{R}_{1}=\mathrm{R}_{2}=\mathrm{O} & \mathbf{3 3 3} \mathrm{R}_{1}=\mathrm{Tig} ; \mathrm{R}_{2}=\mathrm{CH}_{3}\end{array}$<smiles>C=C[C@H]1CC2=C(C)C(=O)N[C@H]2C[C@]1(C)C=C</smiles>

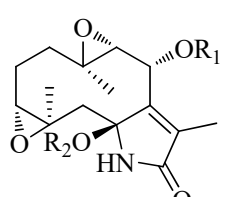<smiles>C/C=C(\C)C(=O)C(C)=O</smiles>

Figure 8. The structures of compounds 325-337.

Apupukeanane-type sesquiterpenoid isomers, 9-thiocyanatopupukeanane isomers (349-350) (Figure 9) were isolated from the the Thai sponge Halichondria sp. [65]. A bioassay-guided phytochemical study was conducted on the semi-mangrove plant Myoporum bontioides. A. Gray, which led to the isolation of two new farnesane sesquiterpene alkaloids, myoporumines A (351) and B (352) (Figure 9), which displayed potent anti-MRSA activity with MIC value of $6.25 \mu \mathrm{g} / \mathrm{mL}$ [83]. Two aromadendrane sesquiterpene 1-isothiocyanatoaromadendrane (353) and 347, one spioaxane-type sesquiterpenoid axamide-3 (354), and two pupukeanane-type sesquiterpenoids $(349,350)$ (Figure 9), were isolated from the nudibranchs Phyllidiella pustulosa and Phyllidia coelestis [67]. 


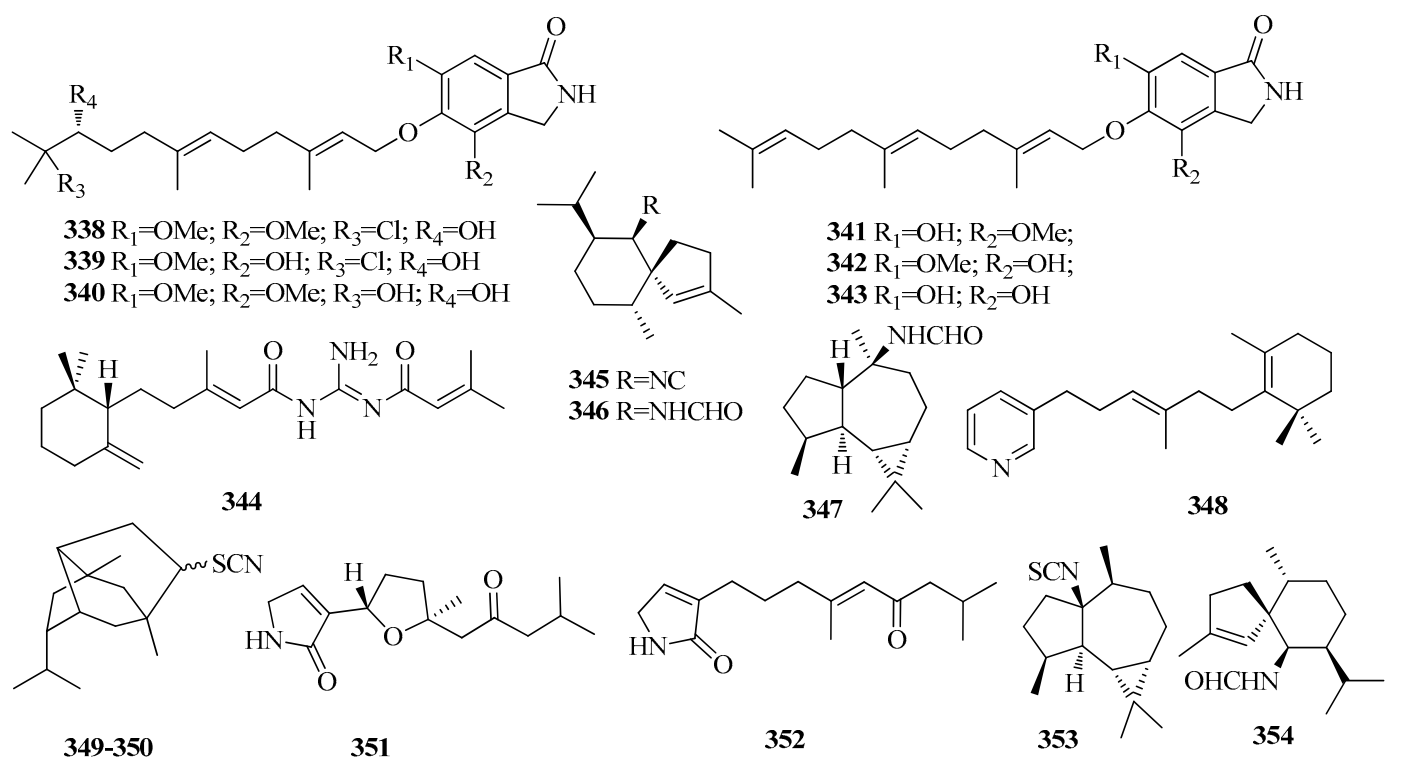

Figure 9. The structures of compounds 338-354.

\subsection{Tremulane, Daucane, Brasilane, Salvialane, Aristolane, Bergamotane and Valerane Sesquiterpenoids}

Huptremules A-D (compounds 355-358) (Figure 10) featuring unusual sesquiterpenoid-alkaloid hybrid structures that integrate the characteristics offungal metabolites (tremulane sesquiterpenoids) and the exogenous substrate, were isolated from a fungal endophyte of Huperzia serrata [84]. Compound 355-358 selectively inhibited acetylcholinesterase activities, with $\mathrm{IC}_{50}$ values of 0.99 , 2.17, 0.11 and $0.06 \mu \mathrm{M}$, respectively [84]. Two daucane-type sesquiterpenoids, aculeneA (359) and B (360) (Figure 10), were identified from Aspergillus aculeatus, which were tested for antifungal activity against Candida albicans. However, all showed only weak orno activity [85]. One brasilane-type sesquiterpenoid, named diaporol L (361) (Figure 10) was isolated from Diaporthe sp., an endophytic fungus associated with the leaves of Rhizophora stylosa collected in Hainan Province, China [86]. One salvialane-type sesquiterpene halichon E (362) and one aristolane sesquiterpene epipolasin A (363) (Figure 10) were obtained from the Thai sponge Halichondria sp. [65]. Sporulaminals A (364) and B (365) (Figure 10), a pair of unusual epimericspiroaminal derivatives, bearing 6/4/5/5 tetracyclic ring system derived from bergamotane sesquiterpenoid, were isolated from a marine-derived fungus Paraconiothyrium sporulosum YK-03 [87]. Volvalerine A (366) (Figure 10), a novel N-containing valerane bisesquiterpenoid derivative with a dihydroisoxazole ring, was isolated from the roots of Valeriana officinalis var. latifolia [88]. Compound 366 was also evaluated for their enhancing activity on NGF mediated neurite outgrowth in PC12 cells. The result indicatedthat the proportion of the NGF-induced neurite-bearing cells (with NGF $5 \mathrm{ng} / \mathrm{mL}$ ) was not enhanced by compound 366 at $50 \mu \mathrm{M}[88]$. 


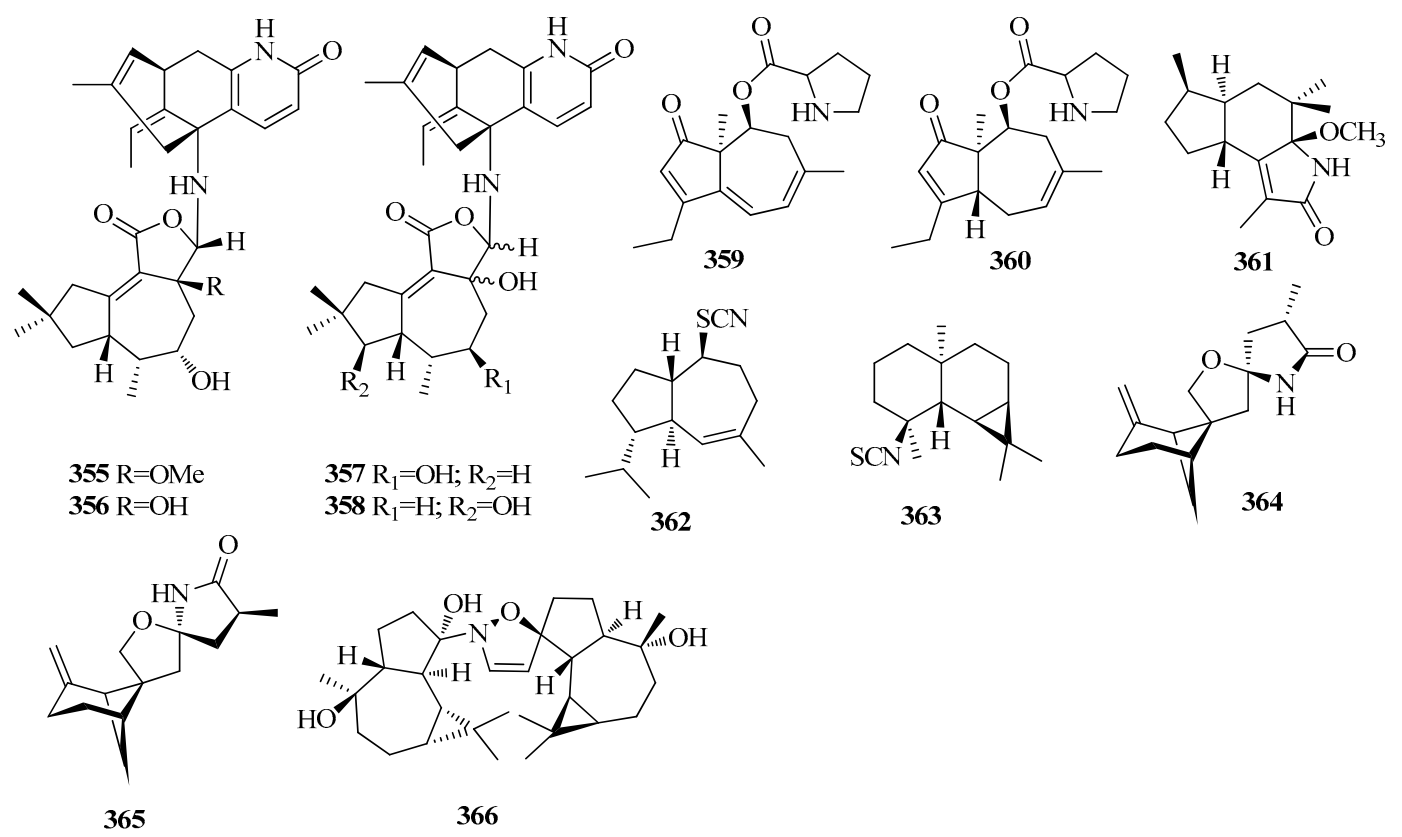

Figure 10. The structures of compounds 355-366.

\subsection{Cyclonerane, Axane, Nardosinane, Zizaane, Eremophilane, and Guaiane Sesquiterpenoids}

The nitrogenous cycloneranesesquiterpenescyclonerin A (367) and B (368) along with seven new congeners-deoxycyclonerins A-D (369-372), cyclonerinal (373), and cyclonerizole (374) (Figure 11) - were isolated from the culture of a marine algicolous strain(A-YMD-9-2) of Trichoderma asperellum [89]. And, compounds (367-374) showed significant cytotoxic activityagainst harmful microalgae Chattonella marina with the $\mathrm{IC}_{50}$ value of $2.1-30 \mu \mathrm{g} / \mathrm{mL}$ [89]. Antartin (375) (Figure 11), a cytotoxic zizaane-type sesquiterpenoid was obtained from a Streptomyces sp. SCO736, isolated from an Antarctic marine sediment, and showed cytotoxicity against A549, H1299, and U87 cancer cell lines by causing cell cycle arrest at the G1 phase [90]. One eremophilane sesquiterpene dendryphiellin J (376) (Figure 11) was isolated from the marine-derived fungus Cochliobolus lunatus SCSIO41401 [91]. Compound 376, a rare naturally occurring aldoxime analogue, displayed cytotoxicities against ACHN and HepG-2 cells with $\mathrm{IC}_{50}$ values of 3.1 and $5.9 \mu \mathrm{M}$, respectively [91]. One unusual sesquiterpenoid dimer, nardochinoid B (377) (Figure 11) was isolated from Nardostachys chinensis Batal [92]. Compound 377 is the first nitrogen-containing nornardosinane-aristolane sesquiterpene conjugate. The $\mathrm{ED}_{50}$ of compound 377 on the production of $\mathrm{NO}$ was 5.73 , and obviously inhibited LPS-inducediNOS and COX-2 protein expression in a dose-dependent way, and increased HO-1 protein expression at the concentration of $10 \mu \mathrm{M}$ [92].Three axane sesquiterpenoid isonitrile pictaisonitrile-1 (378), pictaisonitrile-2 (379), and cavernothiocyanate (380) (Figure 11) were extracted from hyllidiapicta collected from Bali, Indonesia [71]. Vlasoulamine A (381) (Figure 11), an unprecedented guaiane sesquiterpene lactone dimerfeaturing a fully hydrogenated pyrrolo[2,1,5-cd] indolizine core, was isolated from the roots of Vladimiria souliei [93]. Moreover, 381 exhibited neuroprotective activity whenevaluated for glutamate-induced cytotoxicity, nuclear Hoechst 33,258 staining, and measuring intracellular reactive oxygen species levels, using a rat pheochromocytoma PC12 cell-based model system [93]. Clavukoellians A-D (382-385) (Figure 11), highly rearranged nardosinane Sesquiterpenoids with antiangiogenic activity were purified from the marine soft coral Clavularia koellikeri [94]. Compound 382 has aunique skeleton with both lactone and maleimide ring systems, which is rare in natural products, and appears to be formed byoxidative cleavage of the C-7/C- 8 bond of a nardosinane precursor with inhibiting the migration of the human umbilical veinendothelial cells (HUVECs) at $2.5 \mu \mathrm{M}$ [94]. 
<smiles>[R]N(C(=O)/C=C(/C)CCO)C(CCC(O)[C@H]1CCC(C)(O)[C@H]1C)C(=C)C</smiles>

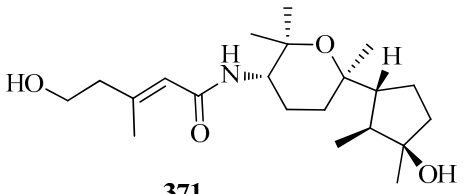

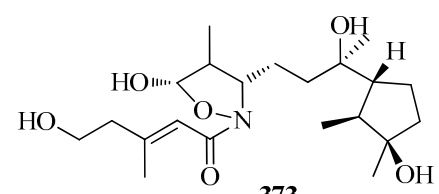

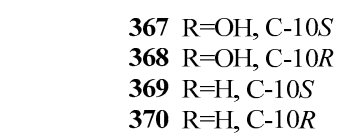<smiles>C/C(=C\C(=O)NC(C)(C)/C=C/CC(O)[C@H]1[C@H](C)CC[C@@]1(C)O)CCO</smiles>

373<smiles>Cc1conc1CCC(O)[C@H]1CCC(C)(O)C1C</smiles>

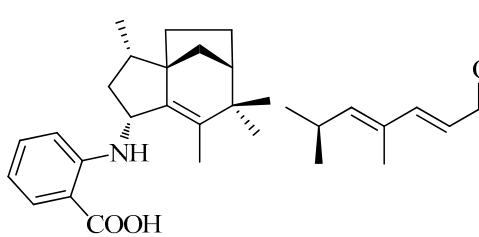

375

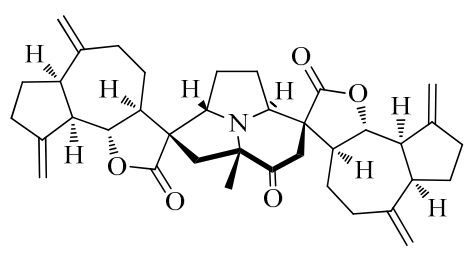

381<smiles>CNCOC(=O)C1=C(C)C(=O)NC1=O</smiles>

382

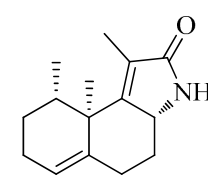

383

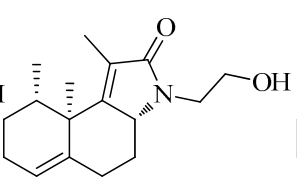

384

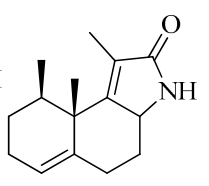

385

Figure 11. The structures of compounds 367-385.

\subsection{Others}

Five sesquiterpene isocyanides, isothiocyanates, thiocyanates, andformamides-halichon A (386), halichon B (387), halichon F (388), halichon H (389), and (+)-2-thiocyanatoneopupukeanane (390) (Figure 12)—were isolated from the Thai sponge Halichondria sp. [65]. Lamellodysidine B (391) (Figure 12), a sesquiterpenes isolated from the marine sponge Lamellodysidea herbacea, collected inIndonesia [95]. Biological activities of 391 was tested in our in-house screening including cytotoxicity, antimicrobial activities, inhibitory activity of the cholesterol ester accumulation in macrophages, inhibitory activity of the RANKL-induced formation of multinuclear osteoclasts, and inhibitory activities of the ubiquitin-proteasome system (proteasome, E1,Ubc13 (E2)-Uev1A interaction, p53-Mdm2 (E3) interaction, and USP7). However, no significant activity was detected forthe compound [95].

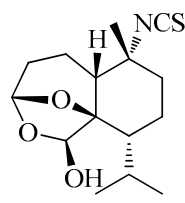

386

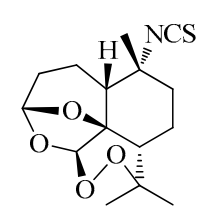

387

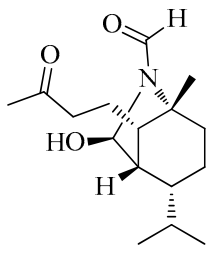

388

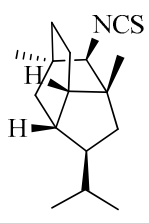

389

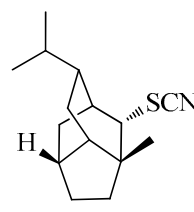

390

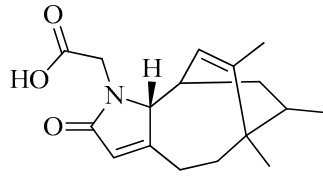

391

Figure 12. The structures of compounds 386-391.

\section{Occurrence}

Natural nitrogenous sesquiterpenoids are mainly distributed in species of plants belonging to the Celastraceae, Saxifragaceae, Zingiberaceae, Asteraceae, Burseraceae, Phyllanthaceae, Magnoliaceae, Lamiaceae, Myoporaceae, and Valerianaceae families, marine sponges belonging to the Dysiseidae, Thorectidae, Spongiidae, and Halichodriae families, soft corals belonging to the 
Xeniidae and Clavulariidae families, phyllidid nudibranchs belonging to the Phyllidiidae family, marine slugs belonging to the Dotidae family), fungi belonging to the Trichocomaceae, Eurotiaceae, Parmulariaceae, Phanerochaetaceae, Diaporthaceae, and Pezizaceae families, bacteria belonging to the Pseudomonadaceae family, and actinomyces belonging to the Streptomycetaceae family (Table 3). Dihydroagarofuran sesquiterpenoids have been isolated from the roots of Maytenus mekongensis, the stems of M. oblongata, the leaves of M. spinosa, the roots and leaves of Tripterygium wilfordii, the stems of T. regelii, the root barks of T. hypoglaucum, the fruits of Celastrus orbiculatus, the seeds of $C$. paniculatus, the root barks of $C$. angulatus, the stems of Euonymus alatus, the whole plants of Parnassia wightiana, the leaves of Monimopetalum chinense. Friedo-drimane and drimane sesquiterpenes have been extracted from maring sponges of the following species: Dysidea sp., D. avara, D. fragilis, D. cinerea, D. septosa, Dactylospongia sp., D. elegans, and D. metachromia. Drimane sesquiterpenoids have been purified from the fungi Aspergillus ochraceus, A. aculeatus, Talaromyces minioluteus, and Penicillium sp. ZZ1283, the bacterium Saccharomonospora sp. CNQ-490, and the actinomycete Streptomyces sp. Eudesmane sesquiterpenoids have been identified inmarine sponges of Halichondria sp., H. okadai, Axinyssa sp., and A. variabilis, the soft coral Cespitularia taeniata, phyllidid nudibranchs of the Phyllidiella sp., P. pustulosa, and P. ocellate species and the plants Curcuma phaeocaulis and Inula helenium L. Germacranese squiterpenoids were isolated from the plants Onopordum alexandrinum, Magnolia kobus, and Salvia scapiformis. Cadinane sesquiterpenes were extracted from the plant Resina commiphora, marine sponges like Halichondria sp. and Axinyssa sp., phyllidid nudibranchs of the Phyllidiella sp. Bisabolane sesquiterpenoids have been isolated from Phyllanthus acidus (L.) skeels, Halichondria sp. Phyllidiella sp., Paraconiothynium brasiliense and P. sporulosum.

Table 3. The species containing nitrogenous sesquiterpenoids.

\begin{tabular}{|c|c|c|c|c|}
\hline Classification & Family & Species & Type & Reference \\
\hline \multirow{20}{*}{ Plant } & \multirow{8}{*}{ Celastraceae } & Maytenus & \multirow{8}{*}{ Dihydroagarofuran } & \\
\hline & & mekongensis; $M$. & & {$[17,25,30]$} \\
\hline & & spinosa; M. oblongata & & \\
\hline & & $\begin{array}{c}\text { Tripterygium wilfordii; } \\
\text { T. regelii; } T .\end{array}$ & & $\begin{array}{c}{[18,20,21,23,24,26,} \\
28,29,31-33,36]\end{array}$ \\
\hline & & $\begin{array}{c}\text { hypoglaucum } \\
\text { Celastrus orbiculatus; }\end{array}$ & & \\
\hline & & $\begin{array}{l}\text { C. angulatus; } C \text {. } \\
\text { paniculatus }\end{array}$ & & {$[19,34,37]$} \\
\hline & & Euonymus alatus & & [22] \\
\hline & & $\begin{array}{l}\text { Monimopetalum } \\
\text { chinense }\end{array}$ & & [35] \\
\hline & \multirow{3}{*}{$\begin{array}{l}\text { Saxifragaceae } \\
\text { Zingiberaceae }\end{array}$} & Parnassia wightiana & & [27] \\
\hline & & Curcuma phaeocaulis & Eudesmane; Elemene & [63] \\
\hline & & Inula helenium L. & Eudesmane & [68] \\
\hline & \multirow[t]{2}{*}{ Asteraceae } & $\begin{array}{l}\text { Onopordum } \\
\text { alexandrinum }\end{array}$ & Germacrane; Elemene & [79] \\
\hline & & Vladimiria souliei & Guaiane & [93] \\
\hline & Burseraceae & Resina commiphora & Cadinane & [72] \\
\hline & Phyllanthaceae & $\begin{array}{c}\text { Phyllanthus acidus (L.) } \\
\text { skeels }\end{array}$ & Bisabolane & [75] \\
\hline & \multirow{3}{*}{$\begin{array}{l}\text { Magnoliaceae } \\
\text { Lamiaceae } \\
\text { Myoporaceae }\end{array}$} & Magnolia kobus & Germacrane & [76] \\
\hline & & Salvia scapiformis & Germacrane & [78] \\
\hline & & Myoporum bontioides & Farnesane & [83] \\
\hline & \multirow{2}{*}{ Valerianaceae } & Valeriana officinalis & Valerane & [88] \\
\hline & & Nardostachys chinensis & Nornardosinane-aristolane & [92] \\
\hline
\end{tabular}


Table 3. Cont.

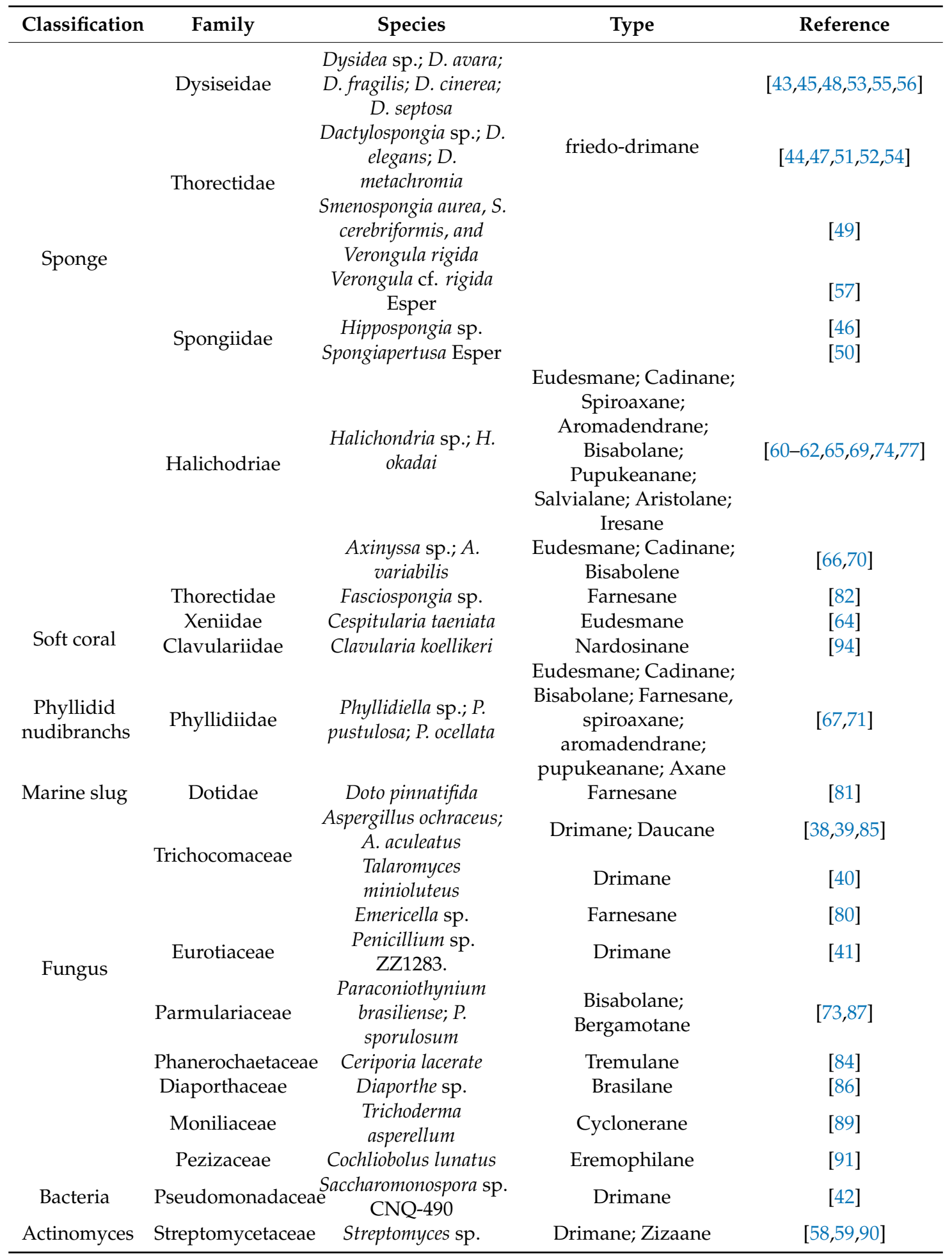

\section{Conclusions}

In summary, a total of 391 bioactive nitrogenous sesquiterpenoids have been isolated and characterized from plants, microorganisms, and marine organisms at the past ten years. This report systematically describes the occurrence, isolation, structures and biological activities ofthese nearly 400 natural products that contain a nitrogen-carbon/nitrogen-nitrogen/nitrogen-sulfurbond. These natural products are dispersed over severalstructural classes, isolated from many different 
sources (bothmarine and terrestrial) and possess a diverse array of biological activities. It can be concluded that the structure types are obviously related to the species sources, and the bioactivities of nitrogenous sesquiterpenoids are obviously related to structure types, being particularly important their cytotoxic activities. The important points arising from this review are the following: (1) There are few structural types of $\mathrm{N}$-containing sesquiterpenes in plants, while the structural types of sesquiterpenes with nitrogen in marine resources and microorganisms are various and diverse. (2) Dihydroagarofuran sesquiterpenoids were considered the most widespread and characteristic metabolites of the plants of Celastraceae, which are well recognized as characteristic metabolitesand important chemotaxonomic markers or indicators of the family, exceptforsome $\beta$-dihydroagarofurans obtained from the Saxifragaceae species Parnassia wightiana. (3) Sponges and their associated microorganisms are the largest contributors of nitrogenous sesquiterpenoids. Rearranged 4,9-friedo-drimaneterpenoid skeletons represent the majority ofnitrogen-contenting sesquiterpenes isolated from marine sponges. The types of sesquiterpenoids that are the most abundant among the marine organisms, Halichondria sp. (sponge) and Phyllidiella sp. (nudibranchs), are all sesquiterpene isocyanides, isothiocyanates, thiocyanates, and formamides. (4) Nitrogenous sesquiterpenes are rich in microorganisms, such as fungus, bacteria and actinomyces and the main skeleton types are drimane, bisabolane, farnesane, tremulane sesquiterpenoids and so on. (5) Dihydroagarofuran sesquiterpenoids show significant anti-inflammatory, neuroprotective, and immunosuppressive effects, while sesquiterpenes isolated from marine organisms exhibit remarkable antitumor cytotoxic activities. Due to the rich activities and structural diversity of N-contenting sesquiterpenes, researchers have not stopped exploring and studying such compounds. We hope this review will stimulate further researchinto this interesting class of nitrogenous secondary metabolites.

Funding: This work was supported in part by Hainan Provincial Natural Science Foundation of China (No. 219MS101), the National Natural Science Foundation of China (No. 81603387), the CAMS Innovation Fund for Medical Sciences (CIFMS) (Nos. 2016-I2M-1-012and 2017-I2M-1-013), the General Programof the Natural Science Foundation of Beijing, China (No. 7082059), and Basic research projects of central-level public welfare research institutes (No. 2018PT35030).

Conflicts of Interest: The authors declare no conflict of interest.

\section{Abbreviations}

OAc<smiles>CC(=O)[OH2+]</smiles>

OBz

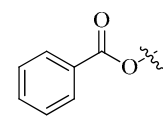

$\mathrm{OFu}$

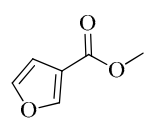

ONic

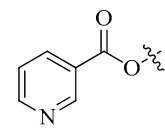


OtCin<smiles>O=C(O)/C=C/c1ccccc1</smiles>

OcCin<smiles>O=C(O)/C=C/c1ccccc1</smiles>

OTig<smiles>CC=CC(=O)OC</smiles>

OMeBut<smiles>CCC(C)C(=O)O</smiles>

\section{References}

1. Zhan, Z.J.; Ying, Y.M.; Ma, L.F.; Shan, W.G. Natural disesquiterpenoids. Nat. Prod. Rep. 2011, $28,594-629$. [CrossRef] [PubMed]

2. Hanson, J. A hundred years in the elucidation of the structures of natural products. Sci. Progress 2017, 100, 63-79. [CrossRef] [PubMed]

3. Bishayee, A.; Sethi, G. Bioactive natural products in cancer prevention and therapy: Progress and promise. Semin. Cancer Biol. 2016, 40, 1-3. [CrossRef]

4. Newman, D.J.; Cragg, G.M. Natural Products as Sources of new drugs over the nearly four decades from 01/1981 to 09/2019. J. Nat. Prod. 2020, 83, 770-803. [CrossRef]

5. Newman, D.J.; Cragg, G.M. Natural products as sources of new drugs over the 30 Years from 1981 to 2010. J. Nat. Prod. 2012, 75, 311-335. [CrossRef]

6. Paterson, I.; Anderson, E.A. The renaissance of natural products as drug candidates. Science 2005, 310, 451-453. [CrossRef] [PubMed]

7. Vasas, A.; Hohmann, J. Xanthanesesquiterpenoids: Structure, synthesis and biological activity. Nat. Prod. Rep. 2011, 28, 824-842. [CrossRef]

8. Ghantous, A.; Gali-Muhtasib, H.; Vuorela, H.; Saliba, N.A.; Darwiche, N. What madesesquiterpene lactones reach cancer clinical trials? Drug Discov. Today 2010, 15, 668-678. [CrossRef]

9. Chadwick, M.; Trewin, H.; Gawthrop, F.; Wagstaff, C. Sesquiterpenoidslactones: Benefits to plants and people. Int. J. Mol. Sci. 2013, 14, 12780-12805. [CrossRef]

10. Alarif, W.M.; Abdel-Lateff, A.; Alorfi, H.S.; Alburae, N.A. Alcyonacea: A potential source for production of nitrogen-containing metabolites. Molecules 2019, 24, 286. [CrossRef]

11. Fraga, B.M. Natural sesquiterpenoids. Nat. Prod. Rep. 2012, 29, 1334-1376. [CrossRef] [PubMed]

12. Fraga, B.M. Natural sesquiterpenoids. Nat. Prod. Rep. 2013, 30, 1226-1264. [CrossRef] [PubMed]

13. Ma, L.F.; Chen, Y.L.; Shan, W.G.; Zhan, Z.J. Natural disesquiterpenoids: An update. Nat. Prod. Rep. 2020. [CrossRef] [PubMed]

14. Liao, S.G.; Yue, J.M. Dimeric sesquiterpenoids. Prog. Chem. Org. Nat. Prod. 2016, 101, 1-112.

15. Blair, L.M.; Sperry, J. Natural products containing a nitrogen-nitrogen bond. J. Nat. Prod. 2013, 76, 794-812. [CrossRef] [PubMed] 
16. Petkowski, J.J.; Bains, W.; Seager, S. Natural products containing a nitrogen-sulfur bond. J. Nat. Prod. 2018, 81, 423-446. [CrossRef] [PubMed]

17. Lhinhatrakool, T.; Prabpai, S.; Kongsaeree, P.; Sutthivaiyakit, S. Antiplasmodial sesquiterpene alkaloids from the roots of Maytenus mekongensis. J. Nat. Prod. 2011, 74, 1386-1391. [CrossRef]

18. Luo, Y.G.; Min, Z.; Qi, Y.; Qiang, P.; Zhang, G.L. Dihydroagarofuran derivatives from the dried roots of Tripterygium wilfordii. J. Nat. Prod. 2012, 75, 98-102. [CrossRef]

19. Xu, J.; Xie, C.F.; Jin, D.Q.; Guo, Y.Q.; Zhao, P.; Wang, S.N.; He, Y.S. Three new dihydroagarofuran sesquiterpenoids from Celastrus orbiculatus. Phytochem. Lett. 2012, 5, 713-716. [CrossRef]

20. Li, C.J.; Xie, F.G.; Yang, J.Z.; Luo, Y.M.; Chen, X.G.; Zhang, D.M. Two sesquiterpene pyridine alkaloids and a triterpenoid saponin from the root barks of Tripterygium hypoglaucum. J. Asian Nat. Prod. Res. 2012, 14, 973-980. [CrossRef]

21. Wang, C.; Li, C.J.; Yang, J.Z.; Ma, J.; Chen, X.G.; Hou, Q.; Zhang, D.M. Anti-inflammatory sesquiterpene derivatives from the Leaves of Tripterygium wilfordii. J. Nat. Prod. 2013, 76, 85-90. [CrossRef] [PubMed]

22. Yan, Z.H.; Han, Z.Z.; Hu, X.Q.; Liu, Q.X.; Zhang, W.D.; Liu, R.H.; Li, H.L. Two new sesquiterpenes from Euonymus alatus. Helv. Chim Acta 2013, 96, 85-92. [CrossRef]

23. Wang, C.; Li, C.J.; Ma, J.; Yang, J.Z.; Chen, X.G.; Hou, Q.; Zhang, D.M. Bioactive sesquiterpene polyol esters from the leaves of Tripterygium wilfordii. Fitoterapia 2014, 96, 103-108. [CrossRef]

24. Luo, Y.G.; Pu, X.; Luo, G.Y.; Zhou, M.; Ye, Q.; Liu, Y.; Gu, J.; Qi, H.Y.; Li, G.Y.; Zhang, G.L. Nitrogen-containing dihydro- $\beta$-agarofuran derivatives from Tripterygium wilfordii. J. Nat. Prod. 2014, 77, 1650-1657. [CrossRef] [PubMed]

25. Fatima, G.N.; Juan, C.O.; Ángel, G.R.; Ana, E.B. $\beta$-Agarofurans and sesquiterpene pyridine alkaloids from Maytenus spinosa. J. Nat. Prod. 2014, 77, 1853-1863.

26. Gao, C.; Huang, X.X.; Bai, M.; Wu, J.; Li, J.Y.; Liu, Q.B.; Li, L.Z.; Song, S.J. Anti-inflammatory sesquiterpene pyridine alkaloids from Tripterygium wilfordii. Fitoterapia 2015, 105, 49-54. [CrossRef]

27. Gao, Z.F.; Zhou, B.H.; Zhao, J.Y.; Cao, F.J.; Zhou, L.; Geng, H.L. Further study on chemical constituents of Parnassia wightiana Wall: Four new dihydro- $\beta$-agarofuran sesquiterpene polyesters. Int. J. Mol. Sci. 2015, 16, 9119-9133. [CrossRef]

28. Fan, D.S.; Zhu, G.Y.; Chen, M.; Xie, L.M.; Jiang, Z.H.; Xu, L.; Bai, L.P. Dihydro- $\beta$-agarofuran sesquiterpene polyesters isolated from the stems of Tripterygium regelii. Fitoterapia 2016, 112, 1-8. [CrossRef]

29. Fan, D.S.; Zhu, G.Y.; Li, T.; Jiang, Z.H.; Bai, L.P. Dimacrolide sesquiterpene pyridine alkaloids from the stems of Tripterygium regelii. Molecules 2016, 21, 1146. [CrossRef]

30. Toure, S.; Nirma, C.; Falkowski, M.; Dusfour, I.; Boulogne, I.; Jahn-Oyac, A.; Coke, M.; Azam, D.; Girod, R.; Moriou, C.; et al. Aedes aegypti larvicidal sesquiterpene alkaloids from Maytenus oblongata. J. Nat. Prod. 2017, 80, 384-390. [CrossRef]

31. Gao, C.; Lou, L.L.; Wang, D.; Zhang, Y.; Huang, X.X.; Song, S.J. Chemical constituents from the roots of Tripterygium wilfordii and their cytotoxic activity. J. Asian Nat. Prod. Res. 2017, 19, 725-731. [CrossRef] [PubMed]

32. Chen, F.Y.; Li, C.J.; Ma, J.; Zhou, J.; Li, L.; Zhang, Z.; Chen, N.H.; Zhang, D.M. Neuroprotective dihydroagarofuran sesquiterpene derivatives from the leaves of Tripterygium wilfordii. J. Nat. Prod. 2018, 81, 270-278. [CrossRef] [PubMed]

33. Chen, F.Y.; Luo, Y.M.; Li, C.J.; Ma, J.; Huang, J.W.; Li, C.; Zhang, D.M. Dihydroagarofuran sesquiterpenoids esterified with organic acids from the leaves of Tripterygium wilfordii. Fitoterapia 2019, 137, 104185. [CrossRef] [PubMed]

34. Chang, X.; Wang, Z.Y.; Chen, X.; Ma, Y.N.; Zhang, H.Y.; Zhao, T.Z. Two new sesquiterpene pyridine alkaloids from root barks of Celastrus angulatus. J. Asian Nat. Prod. Res. 2019, 21, 1043-1051. [CrossRef] [PubMed]

35. Zhao, X.Z.; Yin, M.; Wang, X.Y.; Guo, S.H.; Li, D.L.; Feng, X.; Xu, S. Two new dihydro- $\beta$-agarofuran sesquiterpenes from stems and leaves of Monimopetalum chinense. Chin. Trad. Herbal Drugs 2019, 50, $13-16$.

36. Zhao, P.; Lin, B.; Hou, Z.L.; Zhou, L.; He, Q.J.; Yao, G.D.; Huang, X.X.; Song, S.J. Dihydro- $\beta$-agarofuran sesquiterpenoid derivatives with neuroprotective activity from the leaves of Tripterygium wilfordii. Fitoterapia 2020, 142, 104501. [CrossRef]

37. Fu, Y.F.; Zhao, W.M. Polyesterified sesquiterpenoids from the seeds of Celastrus paniculatus as lifespan-extending agents for the nematode Caenorhabditis elegans. J. Nat. Prod. 2020, 83, 505-515. 
38. Fang, W.; Lin, X.P.; Zhou, X.F.; Wan, J.T.; Lu, X.; Yang, B.; Ai, W.; Lin, J.; Zhang, T.Y.; Tu, Z.C.; et al. Cytotoxic and antiviral nitrobenzoyl sesquiterpenoids from the marine-derived fungus Aspergillus ochraceus Jcma1F17. Med. Chem. Commun. 2014, 5, 701-706. [CrossRef]

39. Tan, Y.H.; Yang, B.; Lin, X.P.; Luo, X.W.; Pang, X.Y.; Tang, L.; Liu, Y.H.; Li, X.J.; Zhou, X. F Nitrobenzoyl sesquiterpenoids with cytotoxic activities from a marine-derived Aspergillus ochraceus Fungus. J. Nat. Prod. 2018, 81, 92-97. [CrossRef]

40. Ngokpol, S.; Suwakulsiri, W.; Sureram, S.; Lirdprapamongkol, K.; Aree, T.; Wiyakrutta, S.; Mahidol, C.; Ruchirawat, S.; Kittakoop, P. Drimane sesquiterpene-conjugated amino acids from a marine isolate of the fungus Talaromyces minioluteus (Penicillium minioluteum). Mar. Drugs 2015, 13, 3567-3580. [CrossRef]

41. Kaleem, S.; Ge, H.J.; Yi, W.W.; Zhang, Z.Z.; Wu, B. Isolation, structural elucidation, and antimicrobial evaluation of the metabolites from a marine-derived fungus Penicillium sp. ZZ1283. Nat. Prod. Res. 2019, 1680669. [CrossRef] [PubMed]

42. Le, T.C.; Lee, E.J.; Lee, J.; Hong, A.; Yim, C.Y.; Yang, I.; Choi, H.; Chin, J.; Cho, S.J.; Ko, J.; et al. Saccharoquinoline, a cytotoxic alkaloidal meroterpenoid from marine-derived bacterium Saccharomonospora sp. Mar. Drugs 2019, 17, 98. [CrossRef] [PubMed]

43. Utkina, N.K.; Denisenko, V.A.; Krasokhin, V.B. Sesquiterpenoid aminoquinones from the marine sponge Dysidea sp. J. Nat. Prod. 2010, 73, 788-791. [CrossRef] [PubMed]

44. Ovenden, S.P.B.; Nielson, J.L.; Liptrot, C.H.; Willis, R.H.; Tapiolas, D.M.; Wright, A.D.; Motti, C.A. Sesquiterpene benzoxazoles and sesquiterpene quinones from the marine sponge Dactylospongia elegans. J. Nat. Prod. 2011, 74, 65-68. [CrossRef]

45. Hamed, A.N.E.; Wätjen, W.; Schmitz, R.; Chovolou, Y.; Edrada-Ebel, R.A.; Youssef, D.T.A.; Kamel, M.S.; Proksch, P. A new bioactive sesquiterpenoid quinone from the Mediterranean Sea marine sponge Dysideaavara. Nat. Prod. Commun. 2013, 8, 89-92.

46. Kumar, R.; Subramani, R.; Aalbersberg, W. Three bioactive sesquiterpene quinones from the Fijian marine sponge of the genus Hippospongia. Nat. Prod. Res. 2013, 27, 1488-1491. [CrossRef]

47. Daletos, G.; Voogd, N.J.; Müller, W.E.G.; Wray, V.; Lin, W.H.; Feger, D.; Kubbutat, M.; Aly, A.H.; Proksch, P. Cytotoxic and protein kinase inhibiting nakijiquinones and nakijiquinols from the sponge Dactylospongia metachromia. J. Nat. Prod. 2014, 77, 218-226. [CrossRef]

48. Jiao, W.H.; Xu, T.T.; Yu, H.B.; Mu, F.R.; Li, J.; Li, Y.S.; Yang, F.; Han, B.N.; Lin, H.W. Dysidaminones A-M, cytotoxic and NF-kB inhibitory sesquiterpene aminoquinones from the South China Sea sponge Dysidea fragilis. RSC Adv. 2014, 4, 9236-9246. [CrossRef]

49. Hwang, I.H.; Oh, J.; Zhou, W.; Park, S.; Kim, J.H.; Chittiboyina, A.G.; Ferreira, D.; Song, G.Y.; Oh, S.; Na, M.K.; et al. Cytotoxic activity of rearranged drimane meroterpenoids against colon cancer cells via down-regulation of $\beta$-catenin expression. J. Nat. Prod. 2015, 78, 453-461. [CrossRef]

50. Li, J.; Gu, B.B.; Sun, F.; Xu, J.R.; Jiao, W.H.; Yu, H.B.; Han, B.N.; Yang, F.; Zhang, X.C.; Lin, H.W. Sesquiterpene quinones/hydroquinones from the marine sponge Spongiapertusa Esper. J. Nat. Prod. 2017, 80, 1436-1445. [CrossRef]

51. Li, J.; Yang, F.; Wang, Z.; Wu, W.; Liu, L.; Wang, S.P.; Zhao, B.X.; Jiao, W.H.; Xu, S.H.; Lin, H.W. Unusual anti-inflammatory meroterpenoids from the marine sponge Dactylospongia sp. Org. Biomol. Chem. 2018, 16, 6773-6783. [CrossRef] [PubMed]

52. Balansa, W.; Mettal, U.; Wuisan, Z.G.; Plubrukarn, A.; Ijong, F.G.; Liu, Y.; Schäberle, T.F. A new sesquiterpenoid aminoquinone from an Indonesian marine sponge. Mar. Drugs 2019, 17, 158. [CrossRef] [PubMed]

53. Jiao, W.H.; Li, J.; Wang, D.; Zhang, M.M.; Liu, L.Y.; Sun, F.; Li, J.Y.; Capon, R.J.; Lin, H.W. Cinerols, nitrogenous meroterpenoids from the marine sponge Dysideacinerea. J. Nat. Prod. 2019, 82, 2586-2593. [CrossRef] [PubMed]

54. Yu, H.B.; Yin, Z.F.; Gu, B.B.; Zhang, J.P.; Wang, S.P.; Yang, F.; Lin, H.W. Cytotoxic meroterpenoids from the marine sponge Dactylospongia elegans. Nat. Prod. Res. 2019, 1633644. [CrossRef]

55. Luo, X.C.; Li, P.L.; Wang, K.Y.; Voogd, N.J.; Tang, X.L.; Li, G.Q. Cytotoxic sesquiterpenoid quinones from South China Sea sponge Dysidea sp. Nat. Prod. Res. 2019, 1679132. [CrossRef]

56. Gui, Y.H.; Liu, L.; Wu, W.; Zhang, Y.; Jia, Z.L.; Shi, Y.P.; Kong, H.T.; Liu, K.C.; Jiao, W.H.; Lin, H.W. Discovery of nitrogenous sesquiterpene quinone derivatives from sponge Dysidea septosa with anti-inflammatory activity in vivo zebrafsh model. Bioorg. Chem. 2020, 94, 103435. [CrossRef] 
57. Jiso, A.; Kittiwisut, S.; Chantakul, R.; Yuenyongsawad, S.; Putchakarn, S.; Schaberle, T.F.; Temkitthaworn, P.; Ingkaninan, K.; Chaithirayanon, K.; Plubrukarn, A. Quintaquinone, a merosesquiterpene from the yellow sponge Verongula cf. rigida Esper. J. Nat. Prod. 2020, $9 \mathrm{~b} 00886$.

58. Che, Q.; Zhu, T.J.; Qi, X.; Attila, M.; Tibor, K.; Mo, X.M.; Li, J.; Gu, Q.Q.; Li, D.H. Hybrid isoprenoids from a reeds rhizosphere soil derived actinomycete Streptomyces sp. CHQ-64. Org. Lett. 2012, 14, 3438-3441. [CrossRef]

59. Zhang, Q.B.; Mándi, A.; Li, S.; Chen, Y.C.; Zhang, W.J.; Tian, X.P.; Zhang, H.B.; Li, H.X.; Zhang, W.M.; Zhang, S.; et al. N-N-coupled indolo-sesquiterpene atropo-diastereomers from a marine-derived actinomycete. Eur. J. Org. Chem. 2012, 27, 5256-5262. [CrossRef]

60. Suto, S.; Tanaka, N.; Fromont, J.; Kobayashi, J. Halichonadins G-J, new sesquiterpenoids from a sponge Halichondria sp. Tetrahedron Lett. 2011, 52, 3470-3473. [CrossRef]

61. Tanaka, N.; Suto, S.; Ishiyama, H.; Kubota, T.; Yamano, A.; Shiro, M.; Fromont, J.; Kobayashi, J. Halichonadins K and L, new dimeric sesquiterpenoids from a sponge Halichondria sp. Org. Lett. 2012, 14,3498-3501. [CrossRef] [PubMed]

62. Tanaka, N.; Suto, S.; Asai, M.; Kusama, T.; Takahashi-Nakaguchi, A.; Gonoi, T.; Fromont, J.; Kobayashi, J. Halichonadins $\mathrm{M}-\mathrm{Q}$, sesquiterpenes from an Okinawan marine sponge Halichondria sp. Heterocycles 2015, 90, 173-185.

63. Ma, J.-H.; Zhao, F.; Wang, Y.; Liu, Y.; Gao, S.Y.; Ding, L.-Q.; Chen, L.X.; Qiu, F. Natural nitric oxide (NO) inhibitors from the rhizomes of Curcuma phaeocaulis. Org. Biomol. Chem. 2015, 13, 8349-8358. [CrossRef] [PubMed]

64. Wang, S.S.; Cheng, Y.B.; Lin, Y.C.; Liaw, C.C.; Chang, J.Y.; Kuo, Y.H.; Shen, Y.C. Nitrogen-containing diterpenoids, sesquiterpenoids, and nor-diterpenoids from Cespitularia taeniata. Mar. Drugs 2015, 13, 5796-5814. [CrossRef]

65. Prawat, H.; Mahidol, C.; Kaweetripo, W.; Prachyawarakorn, V.; Tuntiwachwuttikul, P.; Ruchirawat, S. Sesquiterpene isocyanides, isothiocyanates, thiocyanates, and formamides from the Thai sponge Halichondria sp. Tetrahedron 2016, 72, 4222-4229. [CrossRef]

66. Li, X.W.; Chen, S.H.; Ye, F.; Mollo, E.; Zhu, W.L.; Liu, H.L.; Guo, Y.W. Axiriabilines A-D, uncommon nitrogenous eudesmane-type sesquiterpenes from the Hainan sponge Axinyssa variabilis. Tetrahedron 2017, 73, 5239-5243. [CrossRef]

67. Wu, Q.H.; Chen, W.T.; Li, S.W.; Ye, J.Y.; Huan, X.J.; Gavagnin, M.; Yao, L.G.; Wang, H.; Miao, Z.H.; Li, X.W.; et al. Cytotoxic nitrogenous terpenoids from two South China Sea nudibranchs Phyllidiella pustulosa, Phyllidia coelestis, and their sponge-prey Acanthella cavernosa. Mar. Drugs 2019, 17, 56. [CrossRef]

68. Cai, Y.S.; Wu, Z.; Zheng, X.Q.; Wang, C.; Wang, J.R.; Zhang, X.X.; Qiu, G.F.; Zhu, K.K.; Cao, S.G.; Yu, J.Q. Spiroalanpyrroids $\mathrm{A}$ and $\mathrm{B}$, sesquiterpene alkaloids with a unique spiro-eudesmanolide-pyrrolizidine skeleton from Inula helenium. Org. Chem. Front. 2020. [CrossRef]

69. Prawat, H.; Mahidol, C.; Wittayalai, S.; Intachote, P.; Kanchanapoom, T.; Ruchirawat, S. Nitrogenous sesquiterpenes from the Thai marine sponge Halichondria sp. Tetrahedron 2011, 67, 5651-5655. [CrossRef]

70. Abdjul, D.B.; Kanno, S.; Yamazaki, H.; Ukai, K.; Namikoshi, M. A dimeric urea of the bisabolene sesquiterpene from the Okinawan marine sponge Axinyssa sp. inhibits protein tyrosine phosphatase 1B activity in Huh-7 human hepatoma cells. Bioorg. Med. Chem. Lett. 2016, 26, 315-317. [CrossRef]

71. Sim, D.C.M.; Mudianta, I.W.; White, A.M.; Martiningsih, N.W.; Loh, J.J.M.; Cheney, K.L.; Garson, M.J. New sesquiterpenoid isonitriles from three species of phyllidid nudibranchs. Fitoterapia 2018, 126, 69-73. [CrossRef] [PubMed]

72. Zhu, S.S.; Qin, D.P.; Wang, S.X.; Yang, C.; Li, G.P.; Cheng, Y.X. Commipholactam A, a cytotoxic sesquiterpenoidal lactam from Resina Commiphora. Fitoterapia 2019, 134, 382-388. [CrossRef] [PubMed]

73. Liu, L.; Chen, X.Y.; Li, D.; Zhang, Y.; Li, L.; Guo, L.D.; Cao, Y.; Che, Y.S. Bisabolane sesquiterpenoids from the plant endophytic fungus Paraconiothyrium brasiliense. J. Nat. Prod. 2015, 78, 746-753. [CrossRef] [PubMed]

74. Raiju, K.; Hitora, Y.; Kato, H.; Ise, Y.; Angkouw, E.D.; Mangindaan, R.E.P.; Tsukamoto, S. Halichonic acid, a new rearranged bisabolene-type sesquiterpene from a marine sponge Halichondria sp. Tetrahedron Lett. 2019, 60, 1079-1081. [CrossRef] 
75. Xin, Y.; Xu, M.; Wang, Y.F.; Zheng, X.H.; Zhu, H.T.; Wang, D.; Yang, C.R.; Zhang, Y.J. Phyllanthacidoid U: A new N-glycosyl norbisabolane sesquiterpene from Phyllanthus acidus (L.) skeels. Nat. Prod. Res. 2020, 1712387. [CrossRef]

76. Park, H.W.; Lee, J.H.; Choi, S.U.; Baek, N.I.; Kim, S.H.; Yang, J.H.; Kim, D.K. Cytotoxic germacranolide sesquiterpenes from the bark of Magnolia kobus. Arch. Pharm. Res. 2020, 33, 71-74. [CrossRef]

77. Ohno, O.; Chiba, T.; Todoroki, S.; Yoshimura, H.; Maru, N.; Maekawa, K.; Imagawa, H.; Yamada, K.; Wakamiy, A.; Suenaga, K.; et al. Halichonines A, B, and C, novel sesquiterpene alkaloids from the marine sponge Halichondria okadai Kadota. Chem. Commun. 2011, 47, 12453-12455. [CrossRef]

78. Wang, C.Y.; Kim, D.; Zhu, Y.K.; Oh, D.C.; Huang, R.Z.; Wang, H.S.; Liang, D.; Lee, S.K. Glechomanamides A-C, germacrane sesquiterpenoids with an unusual $\Delta^{8-7}, 12$-lactam moiety from Salvia scapiformis and their antiangiogenic activity. J. Nat. Prod. 2019, 82, 3056-3064. [CrossRef]

79. Sugimoto, S.; Yamano, Y.; Desoukey, S.Y.; Katakawa, K.; Wanas, A.S.; Otsuka, H.; Matsunami, K. Isolation of sesquiterpene-amino acid conjugates, onopornoids $\mathrm{A}-\mathrm{D}$, and a flavonoid glucoside from Onopordum alexandrinum. J. Nat. Prod. 2019, 82, 1471-1477. [CrossRef]

80. Zhang, G.J.; Sun, S.W.; Zhu, T.J.; Lin, Z.J.; Gu, J.Y.; Li, D.H.; Gu, Q.Q. Antiviral isoindolone derivatives from an endophytic fungus Emericella sp. associated with Aegiceras corniculatum. Phytochem. 2011, 72, 1436-1442. [CrossRef]

81. Putz, A.; Kehraus, S.; Díaz-Agras, G.; Wägele, H.; König, G.M. Dotofide, a guanidine-interrupted terpenoid from the marine slug Doto pinnatifida (Gastropoda, Nudibranchia). Eur. J. Org. Chem. 2011, 3733-3737. [CrossRef]

82. Zhao, Z.B.; Sun, J.Z.; Mao, S.C.; Guo, Y.W. Fasciospyrinadine, a novel sesquiterpene pyridine alkaloid from a Guangxi sponge Fasciospongia sp. J. Asian Nat. Prod. Res. 2013, 15, 198-202. [CrossRef] [PubMed]

83. Dong, L.M.; Huang, L.L.; Dai, H.; Xu, Q.L.; Ouyang, J.K.; Jia, X.C.; Gu, W.X.; Tan, J.W. Anti-MRSA sesquiterpenes from the semi-mangrove plant Myoporum bontioides A. Gray. Mar. Drugs 2018, 16, 438. [CrossRef] [PubMed]

84. Ying, Y.M.; Shan, W.G.; Zhan, Z.J. Biotransformation of huperzine A by a fungal endophyte of Huperzia serrata furnished sesquiterpenoid-alkaloid hybrids. J. Nat. Prod. 2014, 77, 2054-2059. [CrossRef] [PubMed]

85. Petersen, L.M.; Hoeck, C.; Frisvad, J.C.; Gotfredsen, C.H.; Larsen, T.O. Dereplication guided discovery of secondary metabolites of mixed biosynthetic origin from Aspergillus aculeatus. Molecules 2014, 19, 10898-10921. [CrossRef] [PubMed]

86. Chen, C.J.; Liu, X.X.; Zhang, W.J.; Zang, L.Y.; Wang, G.; Seik, W.N.; Tan, R.X.; Ge, H.M. Sesquiterpenoids isolated from an endophyte fungus Diaporthe sp. RSC Adv. 2015, 5, 17559-17565. [CrossRef]

87. Zhang, L.H.; Feng, B.M.; Chen, G.; Li, S.G.; Sun, Y.; Wu, H.H.; Bai, J.; Hua, H.M.; Wang, H.F.; Pei, Y.H. SporulaminalsA and B: A pair of unusual epimericspiroaminal derivatives from a marine-derived fungus Paraconiothyrium sporulosum YK-03. RSC Adv. 2016, 6, 42361-42366. [CrossRef]

88. Wang, P.C.; Ran, X.H.; Luo, H.R.; Ma, Q.Y.; Zhou, J.; Hu, J.M.; Zhao, Y.X. Volvalerine A, an unprecedented $\mathrm{N}$-containing sesquiterpenoid dimer derivative from Valeriana officinalis var. latifolia. Fitoterapia 2016, 109, 174-178. [CrossRef]

89. Song, Y.P.; Miao, F.P.; Yin, X.L.; Ji, N.Y. Nitrogenous cyclonerane sesquiterpenes from an algicolous strain of Trichoderma asperellum. Org. Chem. Front. 2019, 6, 3698-6704. [CrossRef]

90. Kim, D.; Lee, E.J.; Lee, J.; Leutou, A.S.; Shin, Y.H.; Choi, B.; Hwang, J.S.; Hahn, D.; Choi, H.; Chin, J.; et al. Antartin, a cytotoxic zizaane-type sesquiterpenoid from a Streptomyces sp. isolated from an Antarctic marine sediment. Mar. Drugs 2018, 16, 130. [CrossRef]

91. Fang, W.; Wang, J.J.; Wang, J.F.; Shi, L.Q.; Li, K.L.; Lin, X.P.; Min, Y.; Yang, B.; Tang, L.; Liu, Y.H.; et al. Cytotoxic and antibacterial eremophilane sesquiterpenes from the marine-derived fungus Cochliobolus lunatus SCSIO41401. J. Nat. Prod. 2018, 81, 1405-1410. [CrossRef] [PubMed]

92. Shen, X.Y.; Qin, D.P.; Zhou, H.; Luo, J.F.; Yao, Y.D.; Lio, C.K.; Li, H.B.; Dai, Y.; Yu, Y.; Yao, X.S. Nardochinoids A-C, three dimeric sesquiterpenoids with specific fused-ring skeletons from Nardostachys chinensis. Org. Lett. 2018, 20, 5813-5816. [CrossRef] [PubMed]

93. Wu, Z.L.; Wang, Q.; Wang, J.X.; Dong, H.Y.; Xu, X.K.; Shen, Y.H.; Li, H.L.; Zhang, W.D. Vlasoulamine A, a neuroprotective [3.2.2]cyclazine sesquiterpene lactone dimer from the roots of Vladimiria souliei. Org. Lett. 2018, 20, 7567-7570. [CrossRef] [PubMed] 
94. Wang, Q.; Hu, Z.Q.; Luo, X.C.; Liu, J.; Li, G.Q.; Cao, S.G.; Liu, Q.S. Clavukoellians A-F, Highly rearranged nardosinane sesquiterpenoids with antiangiogenic activity from Clavularia koellikeri. J. Nat. Prod. 2019, 82, 1331-1337. [CrossRef] [PubMed]

95. Torii, M.; Kato, H.; Hitora, Y.; Angkouw, E.D.; Mangindaan, R.E.P.; Voogd, N.J.; Tsukamoto, S. Lamellodysidines A and B, sesquiterpenes isolated from the marine sponge Lamellodysidea herbacea. J. Nat. Prod. 2017, 80, 2536-2541. [CrossRef]

(C) 2020 by the authors. Licensee MDPI, Basel, Switzerland. This article is an open access article distributed under the terms and conditions of the Creative Commons Attribution (CC BY) license (http://creativecommons.org/licenses/by/4.0/). 